\title{
((1)
}

AUTARQUIA ASSOCIADA À UNIVERSIDADE DE SÃO PAULO

ESTUDO DE TÉCNICAS ESTATÍSTICAS APLICADAS À DETERMINAÇÃO DE PARÂMETROS NO MÉTODO ko DE ANÁLISE POR ATIVAÇÃO NEUTRÔNICA

Rafael Vanhoz Ribeiro

Dissertação apresentada como parte dos
requisitos para obtenção do Grau de
Mestre em Ciências na Área
de Tecnologia Nuclear-Aplicações

Orientador:

Prof. Dr. Mauro da Silva Dias 
INSTITUTO DE PESQUISAS ENERGÉTICAS E NUCLEARES

Diretoria de Pesquisa, Desenvolvimento e Ensino

Av. Prof. Lineu Prestes, 2242 - Cidade Universitária CEP: 05508-000

Fone/Fax(0XX11) 3133-8908

SÃO PAULO - São Paulo - Brasil

http://www.ipen.br

O IPEN é uma Autaquia vinculada à Secretaria de Desenvolvimento, associada à Universiade de São Paulo e gerida técnica e administrativamente pela Comissão Nacional de Energia Nuclear, órgão do Ministério da Ciência, Tecnologia e Inovação. 


\title{
INSTITUTO DE PESQUISAS ENERGÉTICAS E NUCLEARES
}

Autarquia associada à Universidade de São Paulo

\section{ESTUDO DE TÉCNICAS ESTATÍSTICAS APLICADAS À DETERMINAÇÃO DE PARÂMETROS NO MÉTODO ko DE ANÁLISE POR ATIVAÇÃO NEUTRÔNICA}

\author{
Rafael Vanhoz Ribeiro
}

Dissertação apresentada como parte dos requisitos para obtenção do Grau de Mestre em Ciências na Área de Tecnologia Nuclear - Aplicações

Orientador:

Prof. Dr. Mauro da Silva Dias

Versão Corrigida

Versão Original disponível no IPEN

São Paulo

2017 
"Se toda a humanidade menos um fosse da mesma opinião, e apenas um indivíduo fosse de opinião contrária, a humanidade não teria maior direito de silenciar essa pessoa do que esta o teria, se pudesse, de silenciar a humanidade."

(Stuart Mill) 


\section{AGRADECIMENTOS}

Gostaria de agradecer a todos que ajudaram, direta ou indiretamente, a fazer com que este trabalho se realizasse. Por ordem, agradeço ao professor Luiz Paulo Geraldo, o responsável por me motivar e indicar o local aonde eu tentaria cursar minha pós-graduação. Agradeço ao meu professor e orientador Mauro da Silva Dias, à colega de sala Lívia Barros e ao professor Maurício Moralles, por estarem presentes no momento mais importante para que meu trabalho tenha início. Agradeço também ao Instituto de Pesquisas Energéticas e Nucleares (IPEN-CNEN) pelo espaço e por todo apoio oferecido para que meu trabalho fosse concluído.

Agradeço às irmãs Lyslaine e Camila Sato, por confiarem em mim, oferecendo-me uma hospedagem no local onde moram e por vezes, até, me alimentando. Agradeço à Izabela Motta Pinto por estar comigo durante todas as etapas da minha nova fase de pós-graduação, principalmente no primeiro ano, período no qual era minha única companhia.

Agradeço aos meus familiares por toda ajuda e apoio que puderam me oferecer, seja financeiro ou motivacional. E agradeço, por fim, mais uma vez, aos professores Caren Studer e José Caetano da Silva, pois agora estou abrindo os braços do mundo. 


\title{
ESTUDO DE TÉCNICAS ESTATÍSTICAS APLICADAS À DETERMINAÇÃO DE PARÂMETROS NO MÉTODO $k_{0}$ DE ANÁLISE POR ATIVAÇÃO NEUTRÔNICA
}

\author{
Rafael Vanhoz Ribeiro
}

\begin{abstract}
RESUMO
O presente trabalho procurou aperfeiçoar um código criado para calcular os parâmetros $k_{0}$ e $Q_{0}$, denominado COVAR, adicionando um outro método para a determinação do fator $k_{0}$ e aprimorar a análise de covariância existente, criando uma nova versão: COVAR v4.1. O presente trabalho também desenvolveu um novo método de cálculo para os parâmetros $\alpha$ e vários fatores $k_{0}$ em um único ajuste de mínimos quadrados, por meio de uma metodologia inédita, utilizando matrizes de covariância e todas as incertezas parciais envolvidas. Para aplicação deste método, outro código foi desenvolvido, denominado AKFIT v2.1, no qual possui a capacidade de efetuar dois ajustes, linear e não-linear, na determinação de $\alpha$ e $k_{0}$ para várias irradiações. Foi utilizado um conjunto de dados com irradiações realizadas nos anos de 2008 e 2010, pelo Laboratório de Metrologia Nuclear (LMN) e por meio do reator nuclear IEAR-1, do IPEN-CNEN/SP, correspondendo aos radionuclídeos ${ }^{95} \mathrm{Zr},{ }^{65} \mathrm{Zn},{ }^{69 m} \mathrm{Zn},{ }^{46} \mathrm{Sc},{ }^{140} \mathrm{La}$ e ${ }^{60} \mathrm{Co}$, resultando em 21 conjuntos de dados a serem analisados para verificar o desempenho dos códigos criados. Para COVAR v 4.1, os resultados do cálculo alternativo para 0 fator $k_{0}$ foram próximos dos valores obtidos pelo método original do programa e foram consistentes com a literatura. Para AKFIT v2.1, realizou-se ajustes com ambas irradiações simultâneas e separadas. Os modelos ajustados concordaram com a literatura. O valor de $\alpha$ foi de 0,0025(83), que está de acordo com resultados obtidos anteriormente pelo LMN. As correlações entre os parâmetros $k_{0}$ se comportaram como esperado, com valores menores entre diferentes elementos e maiores entre elementos iguais com diferentes energias e usando o mesmo comparador. Pode-se concluir que os métodos propostos foram capazes de calcular os valores de $k_{0}$, com AKFIT v2.1 sendo uma nova técnica no qual é possível determinar dois parâmetros, $\alpha$ e $k_{0}$, ao mesmo tempo, de modo rápido e preciso. Espera-se que o código AKFIT possa ser aperfeiçoado, adicionando mais parâmetros, como $Q_{0}$ e $f$, tornando-o uma ferramenta de ajuste completo para a determinação de todos os parâmetros essenciais do Método $k_{0}$ de AAN.
\end{abstract}

Palavras-chave: Análise por Ativação Neutrônica, Método $k_{0}$, Método dos Mínimos Quadrados, Matriz de Covariância 


\title{
IMPROVEMENT IN STATISTICAL TECHNIQUES APPLIED TO THE DETERMINATION OF PARAMETERS IN THE ko METHOD OF NEUTRON ACTIVATION ANALYSIS
}

\author{
Rafael Vanhoz Ribeiro
}

\begin{abstract}
The present work aims to improve a code for calculating $k_{0}$ and $Q_{0}$ parameters, called COVAR, adding another method of calculating $k_{0}$ factor and improving the covariance analysis, creating a new version: COVAR v4.1. The present work also aims the development of a new method of calculating the alpha and several $k_{0}$ parameters in a single least square fit, by means of a novel methodology, using covariance matrices and all partial uncertainties. For the calculations applying this new method, another code was developed, called AKFIT v2.1 which performs linear and non-linear fittings for the determination of alpha and $k_{0}$ parameters for several irradiations in different periods. We used a database with irradiations in the years 2008 and 2010 performed at the IEAR-1 nuclear reactor of the IPENCNEN/SP, by the Nuclear Metrology Laboratory (LMN), corresponding to radionuclides ${ }^{95} \mathrm{Zr},{ }^{65} \mathrm{Zn},{ }^{69 m} \mathrm{Zn},{ }^{46} \mathrm{Sc},{ }^{140} \mathrm{La}$ and ${ }^{60} \mathrm{Co}$ and resulting in 21 data sets which were analyzed in order to verify the performance of COVAR4.1 and AKFIT2.1. For COVAR v4.1, the results with the alternative calculation of $k_{0}$ factor were close to the already existing calculation and were consistent with the literature. For AKFIT v2.1 fittings were performed with both irradiations simultaneously and separately. The fitted models agreed with the literature. The $\alpha$ value was $0,0025(83)$, which agrees with previous results obtained by the LMN. The correlations between the parameters $k_{0}$ behaved as expected, with smaller values between different elements and greater correlations between equal elements with different energies and using the same comparator measurement. It can be concluded that the proposed methods were able to calculate the values of $k_{0}$, with AKFIT v2.1 being a new technique in which it is possible to determine two parameters, alpha and $k_{0}$ at the same time, quickly and accurately. It is expected that AKFIT code can be improved by adding more parameters, such as $Q_{0}$ and $f$, by making a complete fitting for the determination of all the main parameters for the $k_{0}$ NAA method.
\end{abstract}

Keywords: Neutron Activation Analysis, $k_{0}$ Method, Least Squares Fitting, Covariance Matrix 


\section{SUMÁRIO}

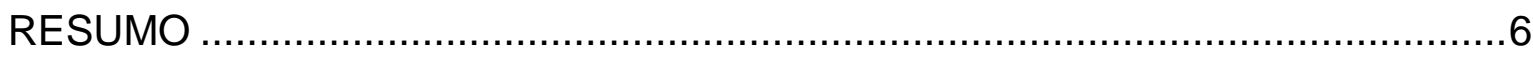

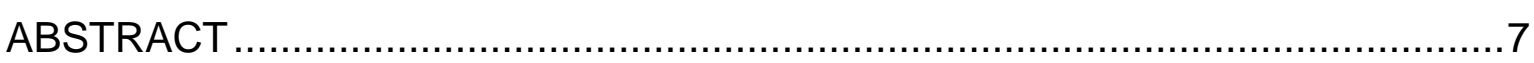

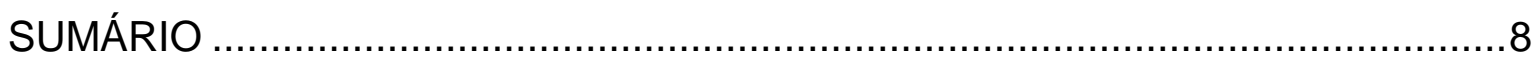

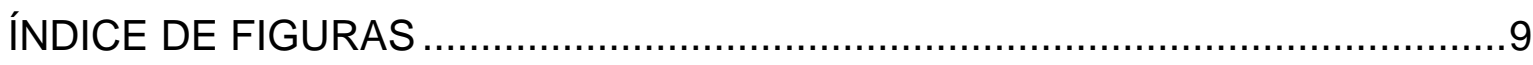

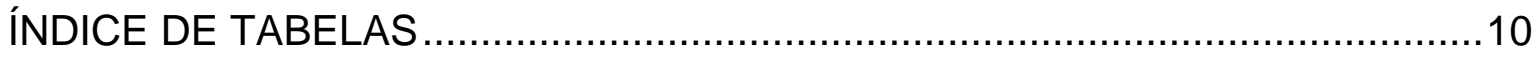

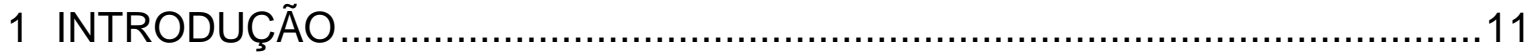

1.1 Objetivos e Justificativa do Presente Trabalho .......................................... 14

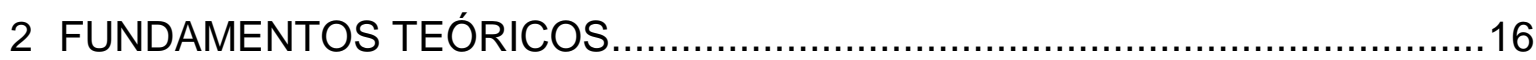

2.1 Interação do nêutron com a matéria ...................................................16

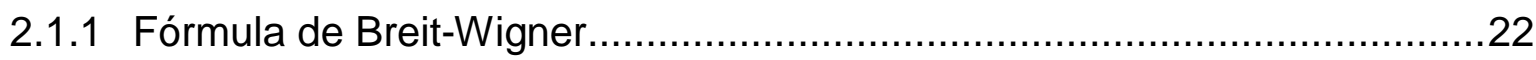

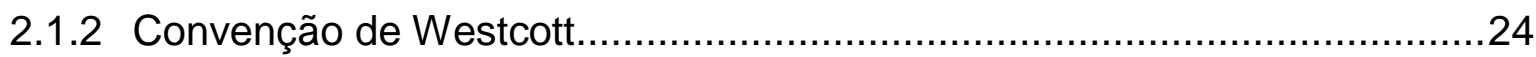

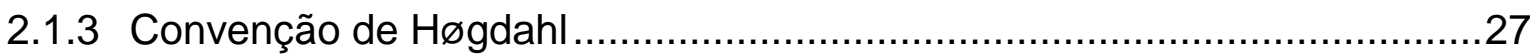

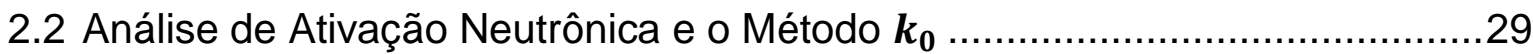

2.3 Determinação de $\boldsymbol{\alpha}$ para o fluxo de nêutrons epitérmicos ............................34

2.3.1 Método da razão de cádmio com monitores múltiplos.............................35

2.3.2 Método de monitores múltiplos cobertos com cádmio ...............................35

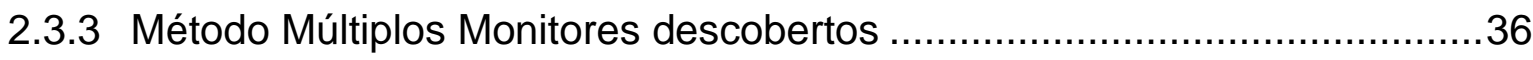

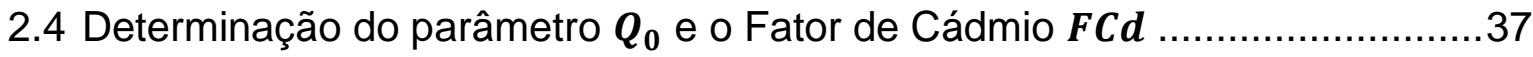

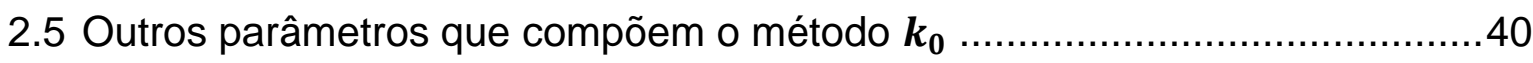

2.5.1 Eficiência de detecção................................................................. 40

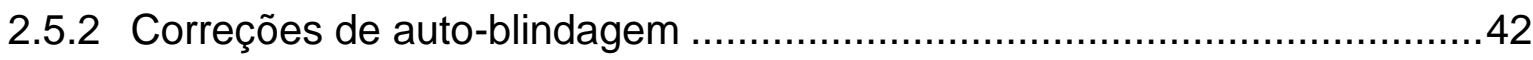

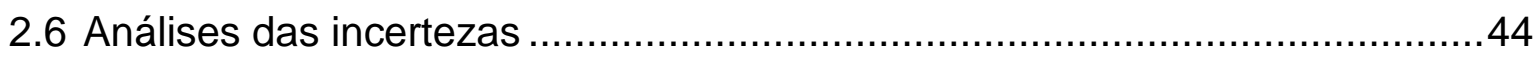

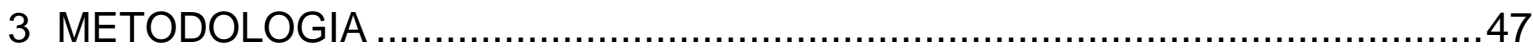

3.1 Atualização do código COVAR ....................................................... 47

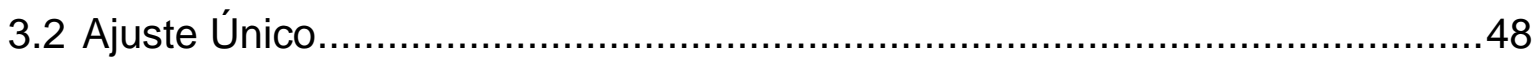

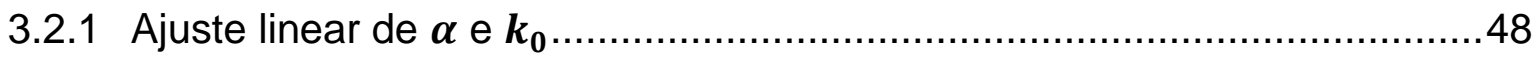

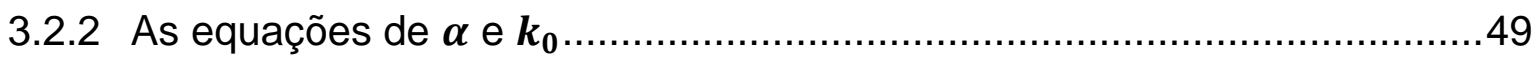

3.2.3 Determinação de $\boldsymbol{\alpha}$ e $\boldsymbol{k}_{\mathbf{0}}$ em um único ajuste .....................................50 


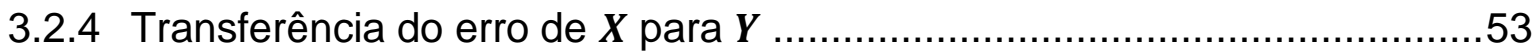

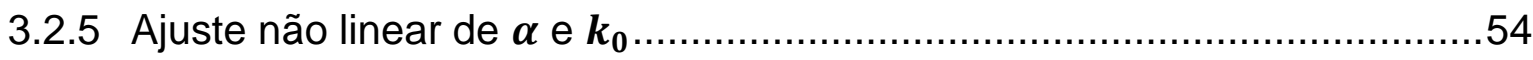

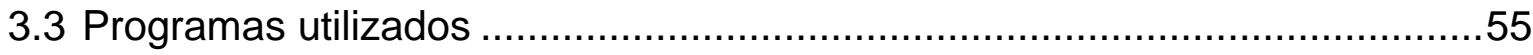

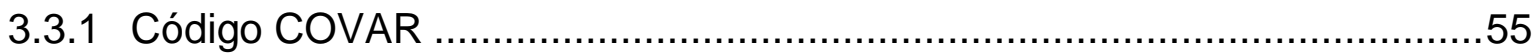

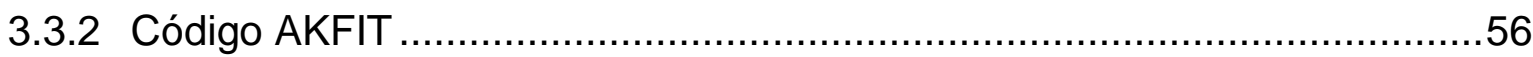

3.3.3 Análises dos espectros e dados experimentais .................................56

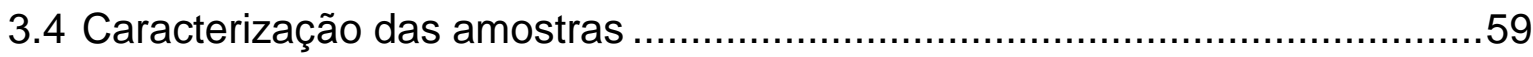

3.4.1 Caracterização das fontes padronizadas e curva de eficiência .................61

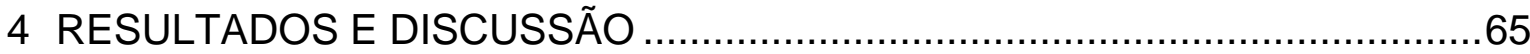

4.1 Curva de eficiência do detector HPGe ................................................65

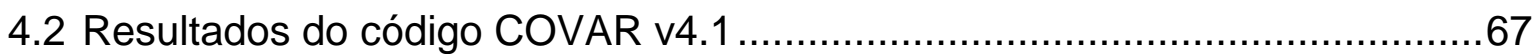

4.3 Resultados do código AKFIT v2.1 .................................................... 71

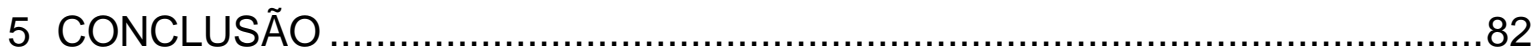

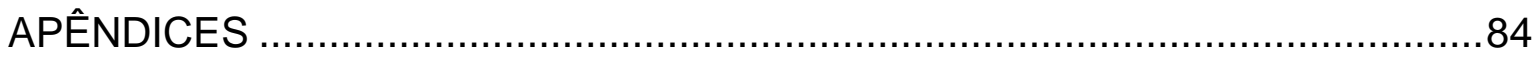

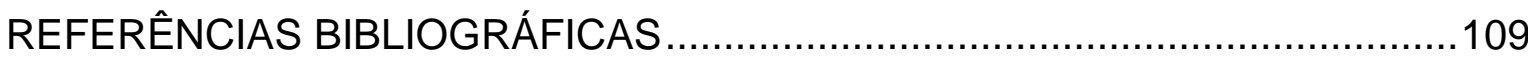




\section{ÍNDICE DE FIGURAS}

FIGURA 1 - Distribuição do fluxo de nêutrons $\varphi(E)$ em função da energia E.

FIGURA 2 - Seção de Choque total do Manganês em função da energia do nêutron.

FIGURA 3 - Seção de choque total de Ródio em função da energia do nêutron com ajuste de Breit-Wigner.

FIGURA 4 - Exemplo dos parâmetros utilizados no método de integração numérica da área sob o pico de absorção total.

FIGURA 5 - Curva de eficiência, em escala log-log, para o espectrômetro de HPGe em função da energia gama, obtida pelas fontes padrões fornecidas pela IUPAC.

FIGURA 6 - Exemplo do resultado obtido na primeira execução do COVAR v4.1. Os números de N (14 e 15) referem-se aos índices atribuídos às transições gama de 724,2 keV e 756,7 keV, da reação ${ }^{94} \mathrm{Zr}(\mathrm{n}, \gamma){ }^{95} \mathrm{Zr}$.

FIGURA 7 - Resultados de $k_{0}$ para os dados de 2008 analisado pelo AKFIT v2.1. Quanto mais próximo de 1, melhor é o resultado.

FIGURA 8 - Resultados de $k_{0}$ para os dados de 2010 analisado pelo AKFIT v2.1. Quanto mais próximo de 1, melhor é o resultado.

FIGURA 9 - Resultados de $k_{0}$ para os dados de 2008 e 2010 analisados simultaneamente pelo AKFIT v2.1. Quanto mais próximo de 1, melhor é o resultado.

FIGURA 10 - Resíduos normalizados do ajuste dos dados de 2008 analisados separadamente.

FIGURA 11 - Resíduos normalizados do ajuste dos dados de 2010 analisados separadamente. .76

FIGURA 12 - Resíduos normalizados do ajuste com ambos os dados de 2008 e 2010 analisados simultaneamente.

FIGURA 13 - Esquema da divisão realizada nas matrizes com suas partes definidas. 


\section{ÍNDICE DE TABELAS}

TABELA 1 - Dados característicos sobre probabilidade de emissão gama, meia-vida, espessura e concentração da liga de cada produto resultante das irradiações no ano de 2008.

TABELA 2 - Dados característicos sobre probabilidade de emissão gama, meia-vida, espessura e concentração da liga das reações dos dados de 2010.

TABELA 3 - Valores das massas e suas incertezas para cada reação nos anos de 2008 e 2010.

TABELA 4 - Características das reações das fontes padronizadas utilizadas para o cálculo da curva de eficiência.

TABELA 5 - Valores de fator de cádmio, correção de auto-blindagem para nêutrons térmicos e epitérmicos, energia de ressonância efetiva, razão entre fluxo térmico e epitérmico, alfa e $Q_{0}$, para as reações de 2008 e 2010

TABELA 6 - Valores das taxas de contagens específicas com e sem Cd para as reações de 2008.

TABELA 7 - Valores das taxas de contagens específicas com e sem cádmio e razão de cádmio para as irradiações de 2010.

TABELA 8 - Dados do ajuste da curva de eficiência do detector HPGe realizado pelo programa LOGFIT.

TABELA 9 - Valores das eficiências das reações.

TABELA 10 - Resultados de $k_{0}$ gerados por meio do COVAR v4.1 utilizando as EQ. (3.1) (K0) e a EQ. (3.3) incluída (K03), para os dados de 2008.

TABELA 11 - Resultados de $\mathrm{k}_{0}$ gerados por meio do COVAR v4.1 utilizando as EQ. (3.1) (K0) e a EQ. (3.3) incluída (K03), para os dados de 2010.

TABELA 12 - Resultados de $Q_{0}$ gerados por meio do COVAR v4.1, para os dados de 2008 e 2010.

TABELA 13 - Resultados dos dados das irradiações do ano de 2008 analisados separadamente. 
TABELA 14 - Resultados dos dados das irradiações do ano de 2010 analisados separadamente.

TABELA 15 - Comparação dos valores de $a, \alpha, \chi 2$ e $\chi$ reduzido 2 entre os ajustes linear e não-linear obtidos pelo código AKFIT v2.1.

TABELA 16 - Comparação dos valores $k_{0}$ entre os ajustes linear e nãolinear obtidos pelo código AKFIT v2.1 .73

TABELA 17 - Representação de cada índice da FIG. 11, com os alvos e suas respectivas transições gama.

TABELA 18 - Comparação entre os valores de $k_{0}$ do código COVAR v4.1 com o método novo e AKFIT v2.1 para os dados de 2008 analisado separadamente.

TABELA 19 - Comparação entre os valores de $k_{0}$ do código COVAR v4.1 com o método novo e AKFIT v2.1 para os dados de 2010 analisado separadamente.

TABELA 20 - Comparação entre os valores de $k_{0}$ do código AKFIT v2.1 com ajuste simultâneo dos dados e do código COVAR v4.1 com o método novo. .80

TABELA 21 - Valores definidos de $k_{0}$ para os elementos analisados .81

TABELA 22 - Comparação dos valores de $k_{0}$ e $Q_{0}$ para ${ }^{64} \mathrm{Zn}$ da literatura com os obtidos no presente trabalho. 
A Análise por Ativação Neutrônica (AAN) é uma técnica mundialmente utilizada para a determinação da concentração de elementos químicos em diferentes materiais ${ }^{[1-3]}$. George Hevesy e Hilde Levi foram os precursores dessa técnica, em um momento em que químicos procuravam uma solução mais eficaz para separar e purificar diferentes elementos dentro de um grupo de terras raras em razão da complexidade que era o processo na época, Hevesy e Levi encontraram a possibilidade de identificar, qualitativa e quantitativamente, elementos que estariam presentes em uma amostra, após verificarem que ao irradiá-la em uma fonte de nêutrons, tornavam-se radioativas, viabilizando a mensuração da radioatividade induzida ${ }^{[1-4]}$.

O conceito empregado pela AAN está na medição da radiação gama produzida após a exposição de uma amostra contendo certa quantidade de elementos em um feixe de nêutrons térmicos por um determinado tempo. Guinn ${ }^{[1]}$ descreve um exemplo prático considerando uma amostra contendo certa quantidade de cobre, na qual estão contidos na mistura os isótopos ${ }^{63} \mathrm{Cu}$ e ${ }^{65} \mathrm{Cu}$, que são estáveis. Ao serem expostos em um feixe de nêutrons térmicos, uma fração do ${ }^{63} \mathrm{Cu}$ tende a capturar um nêutron, tornando-se ${ }^{64} \mathrm{Cu}$, e da mesma maneira, uma fração do ${ }^{65} \mathrm{Cu}$ também irá capturar um nêutron térmico, formando um núcleo de ${ }^{66} \mathrm{Cu}$. Os novos núcleos formados estão em um estado excitado que, para voltarem ao estado fundamental, emitem um raio-gama, conhecido como raio-gama pronto. Se após a exposição ao feixe de nêutrons o núcleo produto da reação $(n, \gamma)$ é um radioisótopo do elemento, ou seja, ele ainda emite radiação gama, pode-se usar esse raio-gama atrasado (delayed gamma ray), para detectar, identificar e medir a quantidade de radiação induzida, em razão de serem característicos do elemento. No caso do cobre, tanto ${ }^{64} \mathrm{Cu}$ quanto ${ }^{66} \mathrm{Cu}$ são radioisótopos do mesmo, o que lhe permite ser usado para os propósitos analíticos.

A aplicação quantitativa da AAN, portanto, é baseada na identificação dos radionuclídeos presentes na amostra pela comparação das linhas de energias 
da emissão gama com as energias da literatura[5]. Esta aplicação é realizada por meio de um método de padronização, que pode ser do tipo relativo, absoluto ou comparador-simples.

O Método Relativo é caracterizado pela irradiação simultânea de uma amostra comparadora certificada e uma amostra que se deseja analisar sob idênticas condições experimentais. Após o processo, as atividades são medidas e, posteriormente, comparadas, proporcionando a quantificação dos elementos. Sua vantagem está na maior precisão obtida, eliminando fontes de incertezas, além de não precisar de um monitoramento da variação do fluxo de nêutrons. Por outro lado, este método necessita de uma amostra padrão para cada elemento analisado, o que demanda muito tempo para irradiação e contagem caso a amostra desconhecida contenha muitos elementos, resultando em um processo dispendioso. Elementos na amostra que não estavam presentes ou não foram certificados na padronização e que aparecerem no espectro da amostra não podem ser determinados. A escolha da amostra de comparação também deve ser feita com cautela, para que diferenças de composição entre a amostra padrão e a desconhecida não acarretem um aumento nas incertezas ${ }^{[6,7]}$. A aplicabilidade deste método, apesar de ser comumente utilizado em razão de suas vantagens, além de ser realizado nas mais diversas condições experimentais e não necessitar de um profundo conhecimento técnico sobre os parâmetros do local de irradiação, é limitada na prática de uma rotina analítica[5].

O Método Absoluto é uma técnica de padronização paramétrica ${ }^{[5,6,8]}$, na qual a concentração do elemento é determinada por parâmetros nucleares e pelo fluxo de nêutrons. Apesar de ser experimentalmente simples e rápida comparada com o Método Relativo, necessita-se de um fluxo de nêutrons contínuo e bem conhecido, ou seja, de um reator estável no qual a variação no fluxo pode ser desprezada. Também é necessário um conhecimento prévio de certos dados nucleares que podem ser grandes fontes de incertezas para a realização deste método ${ }^{[5,7]}$, tais como: as probabilidades de emissão de raiosgama, o fluxo de nêutron e a geometria do sistema, o que o torna menos preciso em razão das incertezas associadas ao grande número de parâmetros ${ }^{[6,8]}$.

O Método $k_{0}$ surgiu como proposta para resolver as desvantagens dos métodos descritos anteriormente, assim como unir as vantagens metodológicas do Método Absoluto com a precisão e exatidão do Método Relativo. Este método 
é descrito em detalhes na seção 2.4 e consiste, basicamente, na irradiação de uma amostra junto com um comparador, onde usualmente é utilizado o ouro para este fim. Desse modo, é possível determinar as concentrações dos elementos por meio da razão entre as atividades do alvo analisado e do comparador, com a vantagem de ser mais versátil e analisar grandes séries de amostras similares com economia no tempo de irradiação, além de resultar em incertezas significativamente menores ${ }^{[5,9-11]}$.

O cálculo da concentração exige um cuidado especial em virtude dos parâmetros que envolvem este método. Alguns dos parâmetros fundamentais são: a razão entre a integral de ressonância e a seção de choque térmica, $Q_{0}$, e a razão entre os fluxos de nêutron térmico e epitérmico, $f$. Segundo Kubešová[6], é muito importante conhecer exatamente os parâmetros do detector e as incertezas associadas a cada um. Os cálculos da incerteza tornam-se complexos em razão da existência de mais parâmetros do que os outros métodos anteriormente citados, além das eventuais correlações existentes entre eles. Smodiš e Bucar ${ }^{[12]}$ demonstraram uma metodologia de medida das incertezas nos resultados obtidos das concentrações pelo método $k_{0}$ com base no Guide to the Expression of Uncertainty and Measurement (GUM). Outros trabalhos para a cálculos das incertezas foram desenvolvidos procurando como objetivo a otimização dos resultados $^{[13-16]}$. Mas apesar de importante para a aquisição de melhores resultados, encontram-se na literatura poucos trabalhos realizados para este fim, ou ainda, não consideram as correlações existente ou todos os parâmetros envolvidos no método. Sgundo Smodiš e Bucar ${ }^{[12]}$, simplificações nos cálculos das incertezas no Método $k_{0}$ devem ser evitadas, pois podem acarretar em erros para os resultados; e na literatura costuma-se à ignorar as correlações existentes.

Nas referências Dias et al. ${ }^{[9,17]}$, foram utilizados um método estatístico para os cálculos das incertezas, empregando matrizes de covariância entre os parâmetros associados. O conceito do método foi tratar as incertezas de forma mais completa, levando em conta a complexidade das correlações existentes entre os parâmetros de ajuste. Desenvolveu-se um código de programação, nomeado COVAR, que estava, até o presente trabalho, na sua versão 3 (COVAR v3), para a realização desses cálculos. Os resultados obtidos pelo código COVAR v3 mostram, entre outras informações, os valores de $k_{0}, Q_{0}$ de cada isótopo analisado e suas respectivas incertezas ${ }^{[18]}$. O código COVAR v3 utiliza uma 
equação de $k_{0}$ que requer que tanto as amostras com o comparador sejam irradiadas com e sem uma cobertura de cádmio (Cd). Conforme apresentado por De Corte ${ }^{[11]}$, existe uma alternativa do cálculo de $k_{0}$ que não requer a irradiação das amostras com cobertura de cádmio. Esta expressão não foi utilizada no código COVAR v3, porém foi incluída no presente trabalho por meio de uma nova versão do código, denominada COVAR v4, sendo assim uma extensão do trabalho de Cardoso ${ }^{[18]}$. Com algumas correções e atualizações no código, sua versão final estabeleceu como COVAR v4.1.

A aplicação do método $k_{0}$ na AAN exige a determinação do parâmetro que descreve a forma real do espectro de nêutrons epitérmico, $\alpha$, além dos parâmetros $k_{0}$ e $Q_{0}$ para cada núcleo-alvo e transição gama utilizada na análise. Existem métodos para determinar o parâmetro $\alpha$ utilizando valores de $k_{0}$ e $Q_{0}$ tabelados e medições de amostras irradiadas com e sem cobertura de cádmio[9].

Moralles, M. ${ }^{[19]}$ elaborou um método de ajuste linear simultâneo para os

parâmetros $\alpha$ e $k_{0}$, denominado AKFIT v1. No presente trabalho, foi implementado o algoritmo do AKFIT v1 e criou-se também o AKFIT v2, que além do ajuste linear, inclui um ajuste não linear para $\alpha$ e $k_{0}$, com base no método de Gauss-Marquardt ${ }^{[20]}$. Uma atualização no código do AKFIT v2 foi necessária para que fosse possível a realização de análises várias irradiações distintas, gerando assim o AKFIT v2.1

\subsection{Objetivos e Justificativa do Presente Trabalho}

O presente trabalho foi motivado pela necessidade de expansão e aprimoramento das metodologias de cálculos dos parâmetros que envolvem o método $k_{0}$, utilizando uma expressão que possibilita a determinação dos valores em um único ajuste, incorporando as incertezas do maior número de parâmetros possível e suas correlações, estendendo a análise de covariâncias desenvolvida por Cardoso e Dias ${ }^{[9,17,18]}$. Este trabalho apresenta aspectos de originalidade associados ao desenvolvimento de uma metodologia teórica, utilizando Matrizes de Covariância em todas as incertezas parciais do método $k_{0}$ para as equações não consideradas anteriormente, e introduzindo uma nova técnica para a determinação simultânea destes parâmetros para vários alvos. O método proposto torna-se particularmente complexo, principalmente na análise e 
determinação da correlação existente entre os parâmetros, pois as equações utilizadas para sua determinação são composições de funções, o que torna a definição da Matriz de Covariância complexa e laboriosa.

O presente trabalho possibilitará uma ampliação na área de pesquisa relacionada com o método $k_{0}$ da AAN do Laboratório de Metrologia Nuclear (LMN) do IPEN, que tem adquirido uma boa experiência no campo de padronização de radionuclídeos e trabalhos com tratamento estatístico dos dados, com a utilização de, entre outros métodos, os de Monte Carlo e regressão. 


\section{FUNDAMENTOS TEÓRICOS}

\subsection{Interação do nêutron com a matéria}

Partículas carregadas leves, como elétrons, e partículas carregadas pesadas, como alfas, interagem com a matéria primariamente através das forças coulombianas (espalhamento de Coulomb) entre a partícula incidente e os elétrons dos átomos do alvo ${ }^{[21]}$. Os nêutrons, entretanto, não possuem carga, consequentemente torna-se impossível a realização desse fenômeno. A interação causada pelos nêutrons, portanto, é através do núcleo alvo de um material. Como resultado dessa interação o nêutron pode desaparecer completamente, emitindose no lugar uma ou mais radiações secundárias (que podem ser partículas pesadas ou raio-gama), ou a sua energia ou direção podem mudar ${ }^{[22]}$. À vista disso, os fenômenos de interação são classificados como:

- Espalhamento elástico $(n, n)$ : a energia cinética total resultante da interação do nêutron incidente com o núcleo alvo se conserva;

- Espalhamento inelástico ( $\left.\mathrm{n}, \mathrm{n}^{\prime}\right)$ : a energia cinética resultante da interação do nêutron e o núcleo alvo não se conserva e o núcleo alvo entra em um estado excitado;

- Captura $(\mathrm{n}, \gamma)$ : o nêutron incidente é absorvido pelo núcleo alvo e emite-se um raio-gama;

- Emissão (n,2n), (n, p), (n, $\alpha$ ) etc.: o nêutron incidente é absorvido pelo núcleo alvo e emite-se um ou mais nêutrons ou uma partícula carregada pesada;

- Fissão (n, f): o núcleo alvo é fissionado, formando novos núcleos atômicos e liberando raios-gama e mais nêutrons.

Usualmente, as reações são representadas como $(n, X)$, na qual $n$ é a radiação incidente e $X$ o resultado das possíveis reações que podem ocorrer. $A$ probabilidade de ocorrer a interação do nêutron com um determinado núcleo alvo altera de acordo com sua energia. Utiliza-se, portanto, essa energia como base para categorizar os nêutrons, que podem ser: nêutrons muito rápidos (entre 15 
MeV a $50 \mathrm{MeV}$ ), rápidos (entre $0,1 \mathrm{MeV}$ a $15 \mathrm{MeV}$ ), intermediários (entre $1 \mathrm{keV}$ a $0.1 \mathrm{MeV}$ ), epitérmicos (entre $1 \mathrm{eV}$ a $1 \mathrm{keV}$ ) e térmicos (inferior a $1 \mathrm{eV}$ ). Para fins práticos, usualmente classificam-se apenas em nêutrons térmicos, epitérmicos e rápidos $^{[23]}$. Na FIG. $1^{[11]}$ é apresentada a distribuição típica em energias de nêutron, para um reator nuclear térmico, como o reator IEA-R1 do IPENCNEN/SP. Pode-se observar a classificação dos nêutrons de acordo com sua energia, em: região dos nêutrons térmicos (também chamada de Distribuição Maxwelliana); região dos nêutrons epitérmicos (que segue aproximadamente a lei $\left.1 / E^{1+\alpha}\right)$ e a região dos nêutrons rápidos (distribuição de Watt).

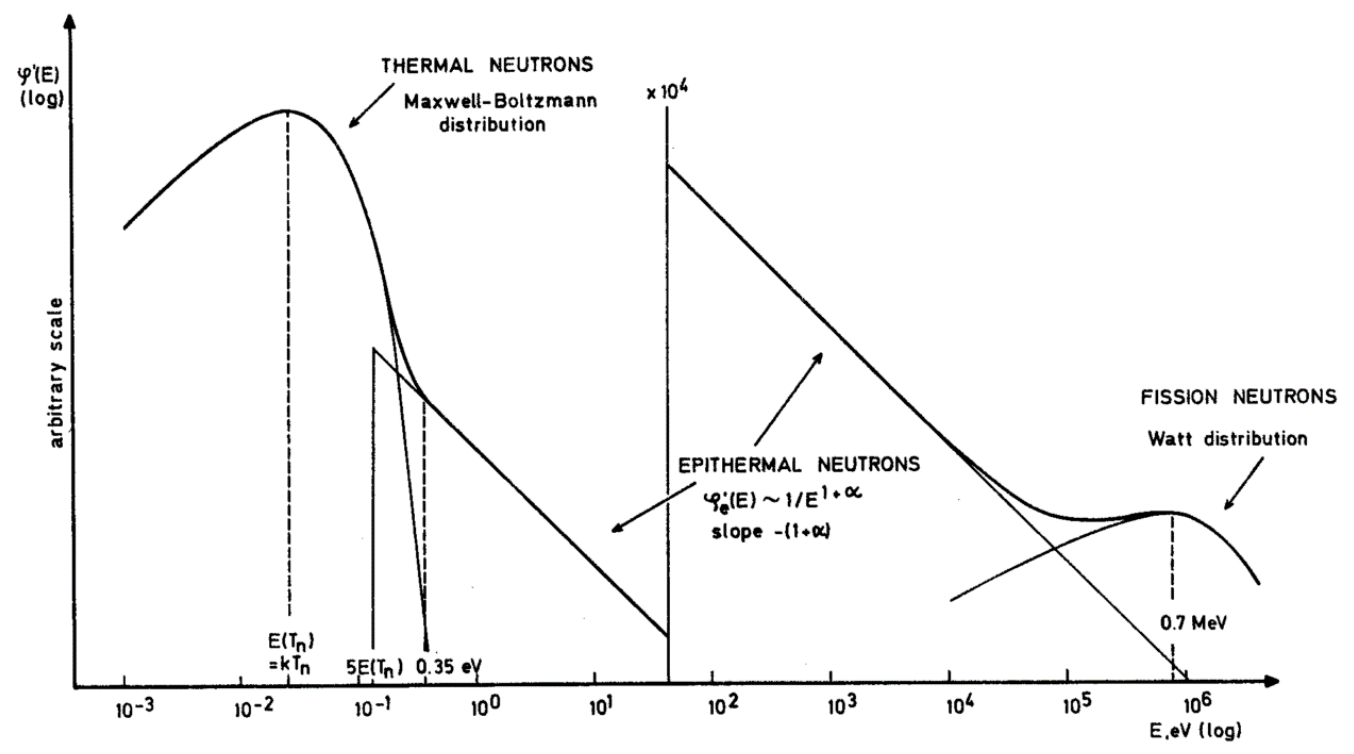

FIGURA 1 - Distribuição do fluxo de nêutrons $\varphi(E)$ em função da energia $E^{[11]}$.

Dentre os tipos de interações para os nêutrons mais lentos, os térmicos (com energias cinéticas abaixo de $1.0 \mathrm{eV}$ ), predominam os fenômenos de espalhamento elástico e captura radiativa. Para nêutrons rápidos predominam as interações por espalhamento elástico, inelástico ou emissão de partícula carregada.

Nêutrons lentos podem atingir o equilíbrio térmico com os núcleos do meio antes que alguma reação nuclear aconteça. Em temperatura ambiente (293 $\mathrm{K}$ ), os nêutrons térmicos possuem uma energia de aproximadamente 0,025 eV. Quando as condições para o equilíbrio térmico são atingidas, os nêutrons possuem uma distribuição Maxwelliana de velocidade, dada por: 


$$
n(v) d v=4 \pi n\left(\frac{m}{2 \pi k T}\right)^{\frac{3}{2}} v^{2} e^{-\frac{m v^{2}}{2 k T}} d v
$$

onde $n$ é o número total de nêutrons por unidade de volume, $n(v) d v$ é o número de nêutrons por unidade de volume que possuem velocidades entre $v$ e $v+d v$, $m$ é a massa de um nêutron, $k$ é a constante de Boltzmann e $T$ a temperatura absoluta do moderador, medida em Kelvin.

Com a distribuição Maxwelliana é possível determinar a velocidade mais provável, diferenciando o lado direito da expressão (2.1) em relação a $v$ e igualando a derivada a zero. Com algumas modificações algébricas pode-se reescrever a EQ. (2.1) como:

$$
n(v)=C f(v)=C v^{2} e^{-\beta^{2} v^{2}}
$$

onde:

$C=4 \pi n\left(\frac{m}{2 \pi k T}\right)^{\frac{3}{2}}$

$\beta=\left(\frac{m}{2 k T}\right)^{\frac{1}{2}}$

De maneira que:

$$
\frac{1}{C} \frac{d n(v)}{d v}=2 v e^{-\beta^{2} v^{2}}-2 v^{3 \beta^{2} e^{-\beta v^{2}}}=0
$$

Para satisfazer a EQ (2.3) em algum valor de velocidade $v=v_{0}$, é necessário que:

$$
v_{0}=\frac{1}{\alpha}=\left(\frac{2 k T}{m}\right)^{\frac{1}{2}}
$$


para cada valor da temperatura absoluta $T$. A velocidade mais provável é quando $v=v_{0}$, em razão de ser o ponto em que $n(v)$ alcança seu valor máximo. A energia cinética correspondente é:

$$
E^{0}=\frac{1}{2} m v_{0}^{2}=k T
$$

O valor numérico da velocidade mais provável $v_{0}$ para temperatura ambiente $(293 \mathrm{~K}$ ) é de $2198 \mathrm{~m} / \mathrm{s}$ (ou, para fins práticos, $2200 \mathrm{~m} / \mathrm{s}$ ), correspondendo a uma energia cinética do nêutron igual a 0,0252 eV. Esses nêutrons são também conhecidos como nêutrons $k T$.

Definem-se como nêutrons epitérmicos, nêutrons que possuem, teoricamente, um espectro do tipo[24, 25]:

$$
\phi_{e}(E)=\Phi_{e} \frac{1}{E}
$$

onde:

$\phi_{e}(E)=$ Intervalo do fluxo epitérmico por unidade de energia do nêutron;

$\Phi_{e}=$ Integral do fluxo epitérmico por unidade de $\ln (E)$

A integral de ressonância para esta condição ideal $\left(\phi_{e}(E) \sim \frac{1}{E}\right)$, que representa a probabilidade de interação entre os nêutrons epitérmicos e os núcleos devido às ressonâncias (pequenas faixas de energia na qual a secção de choque apresenta probabilidade de interação muito maior que a proporcional ao inverso da velocidade) e é dada por:

$$
I_{0}=\int_{E_{C d}}^{\infty} \frac{\sigma(E)}{E} d E
$$

onde:

$E_{C d}=$ Energia efetiva do corte de cádmio

$\sigma(E)=$ Seção de choque microscópica em função da energia 
A seção de choque microscópica, $\sigma$, é a probabilidade de ocorrência de cada um dos fenômenos de interação para nêutrons monoenergéticos incidindo em um núcleo atômico conhecido, e que possui dimensão de área medida em barn. A seção de choque macroscópica, $\Sigma$, resulta da multiplicação de $\sigma$ pelo $N$ números de núcleos atômicos contidos em uma unidade de volume do material alvo[22]:

$$
\Sigma=N \sigma
$$

Já a seção de choque macroscópica total, $\Sigma_{t}$, fornece a probabilidade de ocorrência de todos os fenômenos de interação possíveis, ou seja:

$$
\Sigma_{t}=\Sigma_{e e}+\Sigma_{e i}+\Sigma_{c}+\Sigma_{e}+\Sigma_{f}
$$

onde:

$\Sigma_{e e}=$ Seção de choque macroscópica para espalhamento elástico;

$\Sigma_{e i}=$ Seção de choque macroscópica para espalhamento inelástico;

$\Sigma_{c}=$ Seção de choque macroscópica para captura;

$\Sigma_{e}=$ Seção de choque macroscópica para emissão;

$\Sigma_{f}=$ Seção de choque macroscópica para fissão.

Em determinadas situações, a seção de choque pode aumentar ou diminuir. Isso depende da reação que está sendo verificada e da energia de incidência do nêutron. Para reação $(n, \gamma)$, por exemplo, a seção de choque diminui conforme a energia do nêutron aumenta. Essa proporcionalidade inversa pode ser aproximada para a lei $1 / v$, ou seja, o aumento da taxa de reação é inversamente proporcional à velocidade do nêutron incidente. Também podem ocorrer rápidas elevações no valor da seção de choque em determinadas energias, conhecidas como energia de ressonância, que são causadas quando a formação de um núcleo composto é mais provável.

A energia efetiva do corte de cádmio é definida como 0.55 eV para um detector, possuindo uma seção de choque $\sigma(v)$ proporcional a $1 / v$ para a reação $(n, \gamma)$, irradiado em um fluxo de nêutrons isotrópico com uma pequena amostra 
em um invólucro cilíndrico de cádmio[24]. O conceito empregado de que a distribuição da densidade de fluxo epitérmico varia com o inverso da energia supõe-se válida quando o meio em que os nêutrons estão sendo moderados é homogêneo e infinito; as fontes de nêutrons rápidos estão homogeneamente distribuídas no espaço; o poder de moderação do meio não depende da energia; não existe absorção e as partículas do moderador se comportam como partículas livres com a mesma massa que os nêutrons ${ }^{[26]}$. Entretanto, na prática, essa proporcionalidade de $1 / E$ não é exatamente obedecida. O fluxo de nêutrons epitérmicos em um caso real é corrigido por uma aproximação do tipo:

$$
\phi_{e}(E)=\Phi_{e} \frac{1}{E^{1+\alpha}}
$$

onde $\alpha$, que é independente da energia, pode ser positivo ou negativo, dependendo da configuração do reator ${ }^{[24]}$. A integral de ressonância, definida na EQ. (2.6) e tabelada na literatura, não é válida no caso real. Para um espectro de nêutrons epitérmicos do tipo $1 / E^{1+\alpha}$, a integral de ressonância torna-se:

$$
I_{0}=\int_{E_{C d}}^{\infty} \frac{\sigma(E)}{E^{1+\alpha}} d E
$$

Isso indica que as integrais de ressonância para uso prático são uma função de $\alpha$, e portanto, do local de irradiação considerado. Também implica que para a conversão de $I_{0}$ em $I_{0}(\alpha)$ e vice-versa, deve-se conhecer a relação existente entre elas, enquanto que, para $\alpha$, deve ser determinado experimentalmente ${ }^{[24]}$, como será demostrando da sessão 2.3.

Nêutrons rápidos não são desejáveis em um reator nuclear, em razão de que a maioria dos combustíveis físseis possuem uma alta taxa de reação para fissão e captura para nêutrons lentos. Por este motivo, passam pelo processo de moderação, que consiste em desacelerar nêutrons rápidos por meio de espalhamento elástico de núcleos atômicos até que atinjam o equilíbrio térmico com o meio. Para um bom moderador, as seções de choque para os fenômenos de captura e fissão devem ser pequenas. Caso contrário os nêutrons irradiados serão perdidos por absorção no moderador. 


\subsubsection{Fórmula de Breit-Wigner ${ }^{[27]}$}

A formação de um núcleo composto ocorre quando a soma das energias cinética e de ligação do nêutron incidente corresponde ao estado excitado do núcleo composto, conhecida como reação de ressonância. Em uma situação de ressonância isolada, como no exemplo da FIG. $2^{[27]}$, no qual as ressonâncias são bem separadas por um intervalo de alguns keV por todo o trajeto para altas energias, pode-se descrever a variação da seção de choque de uma reação por meio da fórmula de Breit-Wigner. Considera-se uma largura $\Gamma$ (incerteza de energia) correspondente a cada vida média $\tau$ de um núcleo composto:

$$
\Gamma=\frac{\hbar}{\tau}
$$

A probabilidade de um núcleo composto decair é $\Gamma / \hbar=1 / \tau$. A largura total $\Gamma$ é resultante da soma das larguras parciais para os diversos canais de decaimento $\Gamma_{\gamma}+\Gamma_{n}+\Gamma_{\alpha} \ldots$ A probabilidade, portanto, do núcleo composto decair por meio de uma reação $\Gamma_{i}$ é $\Gamma_{i} / \Gamma$.

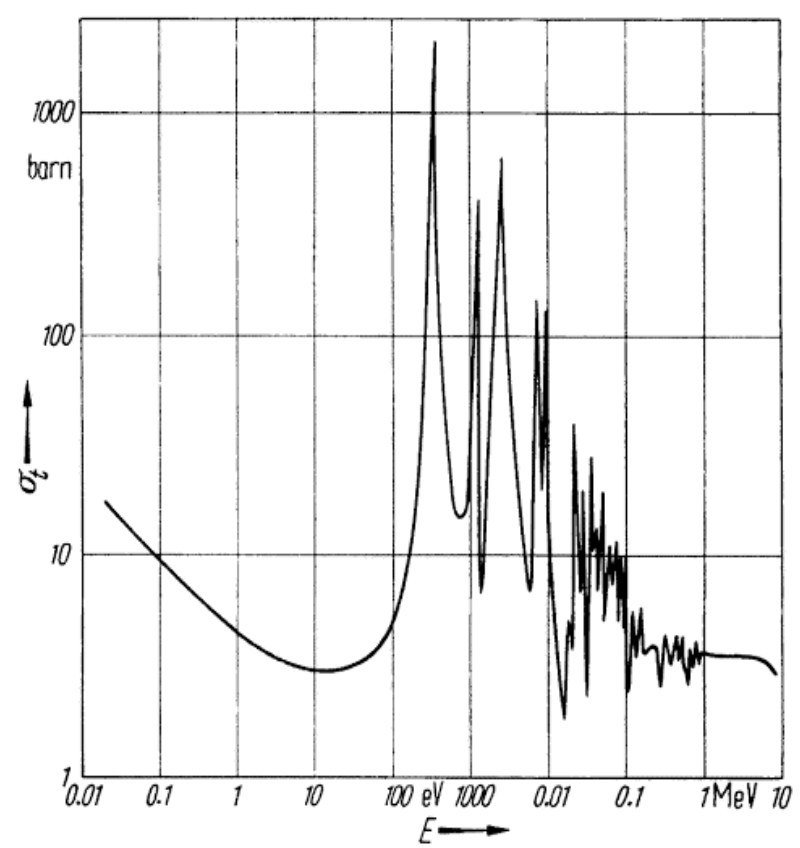

FIGURA 2 - Seção de Choque total do Manganês em função da energia do nêutron. 
A seção de choque para a formação de um núcleo composto por meio de um canal $i$ com um momento angular $l$ pode ser expressa por ${ }^{[28]}$ :

$$
\sigma_{C_{l}}(i)=(2 l+1) \pi \lambda^{2} T_{l}(i)
$$

onde $\sigma_{C_{l}}(i)$ é $\circ$ produto da seção de choque máxima $(2 l+1) \pi \chi^{2}$ com $\circ$ coeficiente de transmissão $T_{l}(i)$.

Entretanto, a EQ. (2.12) não considera os fenômenos de ressonância. Pode-se dizer que é válida em situação na qual $\sigma_{C_{l}}(i)$ está em um intervalo de energia onde se encontra longe o suficiente da região de ressonâncias. Adicionando, portanto, o fenômeno de ressonância, tem-se a expressão:

$$
\sigma_{C_{l}}(i)=(2 l+1) \pi \chi^{2} \frac{\Gamma_{\mathrm{n}} \Gamma}{\left(\mathrm{E}-E_{R}\right)^{2}+\left(\frac{1}{2} \Gamma\right)^{2}}
$$

onde:

$E=$ Energia do nêutron incidente;

$E_{R}=$ Energia de ressonância;

$\lambda=\frac{\lambda}{2 \pi}=$ Comprimento de onda de de Broglie, associado à velocidade do nêutron.

A fim de encontrar a seção de choque de uma determinada reação $(\mathrm{n}, i)$, multiplica-se $\sigma_{C_{l}}(i)$ pela probabilidade de decaimento do núcleo composto por meio do canal $i, \Gamma_{i} / \Gamma$ :

$$
\sigma(n, i)=(2 l+1) \pi \chi^{2} \frac{\Gamma_{\mathrm{n}} \Gamma_{i}}{\left(\mathrm{E}-E_{R}\right)^{2}+\left(\frac{1}{2} \Gamma\right)^{2}}
$$

A EQ. (2.14) é conhecida como fórmula de Breit-Wigner para um único nível. Deve-se notar que os comprimentos $\Gamma_{i}$ são dependentes dos canais de reação, mediante os coeficientes de transmissão $T_{l}(i)$. A FIG. 3 apresenta um exemplo da seção de choque total de Ródio em função da energia do nêutron, com ajuste de Breit-Wigner ${ }^{[27]}$. 


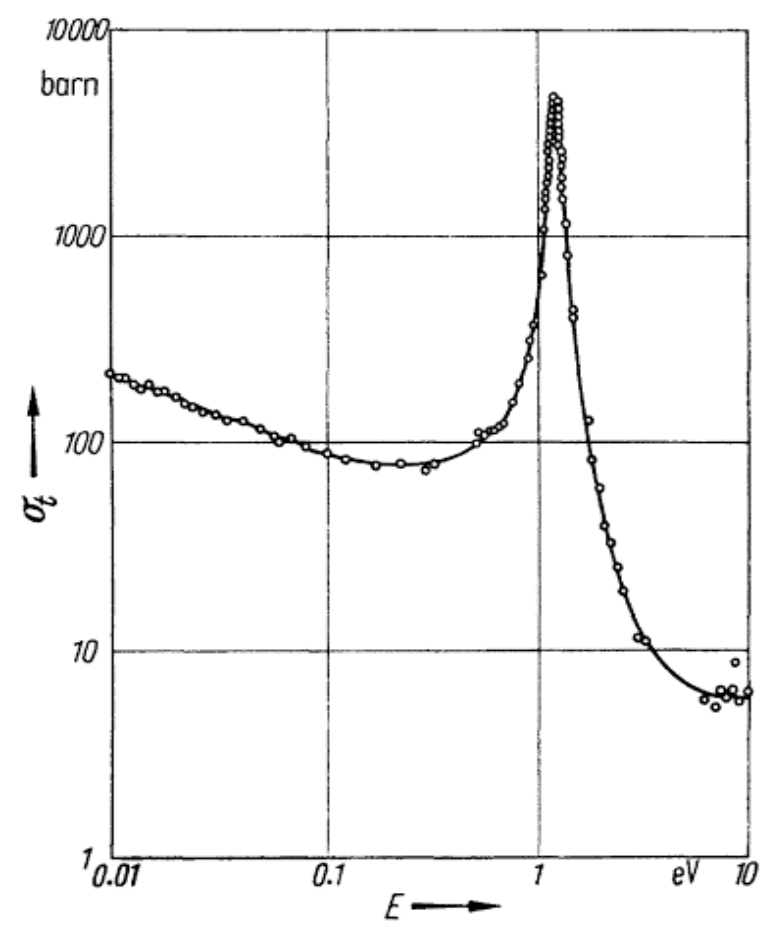

FIGURA 3 - Seção de choque total de Ródio em função da energia do nêutron com ajuste de Breit-Wigner ${ }^{[27]}$.

A fórmula de Breit-Wigner não é adequada quando há influência de outras ressonâncias. Por este motivo, realiza-se um ajuste na EQ. (2.14) no qual considera a contribuição de todas as probabilidades parciais correspondentes de cada ressonância para o valor de energia $E$ do nêutron incidente ${ }^{[29-31]}$.

$$
\sigma(E)=g \pi \lambda^{2} \sum_{i-1}^{n} \frac{\Gamma_{\mathrm{n}} \Gamma_{\mathrm{i}}}{\left(\mathrm{E}-E_{R i}\right)^{2}+\left(\frac{1}{2} \Gamma_{\mathrm{i}}\right)^{2}}
$$

\subsubsection{Convenção de Westcott ${ }^{[32-35]}$}

O formalismo de Westcott, apresentado em 1958, procura solucionar alguns problemas relacionados com a seção de choque de um espectro de nêutron, descrevendo uma distribuição precisa para as seções de choque que não obedecem a lei-1/vi36]. A convenção proposta possui a característica na qual o valor da seção de choque que varia inversamente com a velocidade do nêutron para cada reação (obedece a lei-1/v), corresponde ao próprio valor da seção de choque do nêutron com velocidade de $2200 \mathrm{~m} / \mathrm{s}$. A taxa de reação, neste caso, é proporcional à densidade de nêutrons e é independente do seu espectro. 
Define-se nesta convenção a seção de choque eficaz, $\hat{\sigma}$, e uma taxa de reação por núcleo presente, $R$, sendo o produto de $\hat{\sigma}$ com $n v_{0}{ }^{[34]}$, onde:

$n=$ Densidade do nêutron, incluindo ambos nêutrons térmicos e epitérmicos; $v_{0}=2200 \mathrm{~m} / \mathrm{s}$, assim sendo $R=n v_{0} \hat{\sigma}$.

Quando a seção de choque se afasta da lei-1/v, pode-se obter uma relação aplicando a seção de choque efetiva $\hat{\sigma}$ no espectro de um reator de nêutrons bem termalizado. Supõe-se que o espectro de nêutrons seja a soma de dois componentes: a distribuição do fluxo de nêutrons epitérmicos cortada num limite inferior adequado e uma distribuição Maxwelliana correspondente ao $k T$. A relação, portanto, é:

$$
\hat{\sigma}=\sigma_{0}(g+r s)
$$

onde:

$\sigma_{0}=$ Seção de choque para nêutrons com velocidade de $2200 \mathrm{~m} / \mathrm{s}$;

$g$ e $s=$ Funções da temperatura $T$ que depende do desvio da lei-1/v da seção de choque;

$r=$ Índice que representa a intensidade relativa do componente epitérmico.

Para a lei-1/v, $g=1$ e $s=0$. Quando $r=0$, o espectro é puramente Maxwelliano[33, 37]. A EQ. (2.16) é alcançada considerando que distribuição da densidade de nêutrons, admitindo a suposição, pode ser expressa como:

$$
n(v)=n(1-f) \rho_{m}(v)+n f \rho_{e}(v)
$$

onde:

$f=$ Fração da distribuição epitérmica na densidade total de nêutrons;

$n(1-f)=$ Densidade de nêutrons térmicos;

$n f=$ Densidade de nêutrons epitérmicos.

$\rho_{m}(v)=$ Função normalizada de densidade de probabilidade de nêutrons térmicos por unidade de velocidade, dada por ${ }^{[32]}$ : 


$$
\rho_{m}(v)=\frac{4}{\sqrt{\pi}} \frac{v^{2}}{v_{t_{n}}^{3}} e^{-\left(v \backslash v_{t_{n}}\right)^{2}}
$$

onde o índice $t_{n}$ é a temperatura do nêutron;

$\rho_{e}(v)=$ Função normalizada de densidade de probabilidade de nêutrons epitérmicos por unidade de velocidade, dada por:

$$
\rho_{e}(v)=v_{t_{n}} \sqrt{\mu} \frac{\Delta}{v^{2}}
$$

O parâmetro $\Delta$ é uma função de corte que descreve o término para as menores energias da distribuição epitérmica ${ }^{[38]}$, considerando, no caso da EQ. (2.19), que $\Delta=1$ se $E>\mu k T$ e $\Delta=0$ se $E<\mu k T$.

Com as EQ. (2.17), EQ. (2.18) e EQ. (2.19), é possível escrever a taxa de reação $R$, como definido por meio da seção de choque efetiva, de modo que:

$$
\begin{gathered}
R=n v_{0} \hat{\sigma}=\int_{0}^{\infty} n(v) \sigma(v) v d v \\
R=n(1-f) \int_{0}^{\infty} \frac{4}{\sqrt{\pi}} \cdot \frac{v^{3}}{v_{t_{n}}^{3}} e^{-\left(v \backslash v_{t_{n}}\right)^{2}} \sigma(v) d v+n f \cdot v_{t_{n}} \sqrt{\mu} \cdot \int_{0}^{\infty} \frac{\Delta}{v} \sigma(v) d v
\end{gathered}
$$

Caso o espectro seja puramente Maxwelliano, $f=0$ e a taxa de reação depende somente da primeira integral da EQ. (2.20). A seção de choque $\hat{\sigma}_{m}$ efetiva para este caso é[34]:

$$
\hat{\sigma}_{m}=\frac{1}{v_{0}} \int_{0}^{\infty} \frac{4}{\sqrt{\pi}} \frac{v^{3}}{v_{t_{n}}^{3}} e^{-\left(v \backslash v_{t_{n}}\right)^{2}} \sigma(v) d v
$$

Com a EQ. (2.21) Westcott define o fator $g$ como sendo a razão entre $\hat{\sigma}_{m}$ e $v_{0}{ }^{[32]}$ :

$$
g=\frac{\widehat{\sigma}_{m}}{v_{0}}=\frac{1}{v_{0} \sigma_{0}} \int_{0}^{\infty} \rho_{m}(v) v \sigma(v) d v
$$


O fator $g$ é conhecido como fator de Westcott e permite corrigir a seç̧ão de choque Maxwelliana efetiva para a influência da temperatura. Nas reações $(n, \gamma)$ que não possuem ressonâncias abaixo da energia de corte de cádmio, o fator $g=1$. Existem diversas tabelas com valores de $g$ para elementos de acordo com a temperatura ${ }^{[33,36,37]}$.

Na situação em que $f$ é diferente de zero, a seção de choque efetiva é:

$$
\hat{\sigma}=g \sigma_{0}+\frac{f \sqrt{\mu}}{2} \frac{v_{t}}{v_{0}} \int_{0}^{\infty}\left[\sigma(v)-\frac{v_{0} \widehat{\sigma}_{m}}{v}\right] \frac{\Delta d E}{E}
$$

O parâmetro $r$, conhecido por fração de nêutrons epitérmicos, é expresso sendo proporcional a $f \sqrt{\mu}$ da EQ. (2.23):

$$
r=\frac{f \sqrt{\pi \mu}}{4}
$$

e o fator $s$ também é expresso como:

$$
s=\frac{1}{\sigma_{0}} \sqrt{\frac{4 \mathrm{~T}}{\pi \mathrm{T}_{0}}} \int_{0}^{\infty}\left[\sigma(v)-\frac{v_{0} \widehat{\sigma}_{m}}{v}\right] \frac{d E}{E}
$$

Chega-se, portanto, na relação entre $\sigma_{0}$ e $\hat{\sigma}$, conforme descrito na EQ. (2.16): $\hat{\sigma}=\sigma_{0}(g+r s)$. A taxa de reação dada por:

$$
R=n v_{0} \sigma_{0}(g+r s)
$$

\subsubsection{Convenção de Høgdahl}

Apesar da convenção de Westcott ser precisa, ela é complexa e, na prática, considera-se que apenas alguns radionuclídeos se afastam da lei-1/v para as seções de choque de modo que as incertezas sejam significativamente

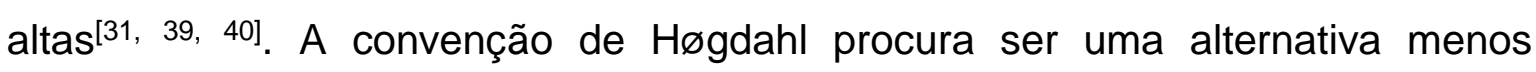
laboriosa, o que torna esta convenção simples para ser utilizada. Entretanto, ela é restrita para reações $(n, \gamma)$ nas quais o fator de Westcott $g$ é igual à unidade ${ }^{[5,40,}$ 
41]. Em situações em que $g$ difere da unidade, recomenda-se não utilizar a convenção de Høgdahl e considerar a temperatura do nêutron, o que, em outras palavras, significa utilizar o formalismo de Westcott ${ }^{[35,42-44]}$. Ao longo dos anos,

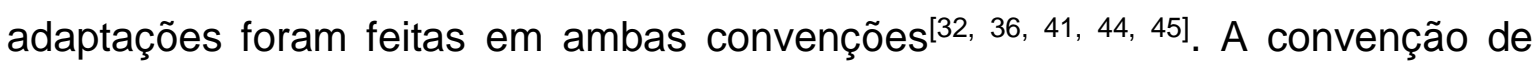
Høgdahl pode ser escrita dividindo a taxa de reação $R$ em duas partes ${ }^{[44]}$ :

$$
R=\int_{0}^{V_{C d}} \sigma(v) \phi(v) d v+\int_{V_{C d}}^{\infty} \sigma(v) \phi(v) d v
$$

Observa-se que $R$ é uma soma das partes térmicas (primeira integral) e epitérmicas (segunda integral). $V_{C d}$ é a velocidade do nêutron correspondente à energia de corte de cádmio. Dessa forma, para reações (n,y) sem ressonâncias abaixo da energia de corte de cádmio $E_{C d}$, a primeira integral é definida como:

$$
\int_{0}^{V_{C d}} \sigma(v) \phi(v) d v=\sigma_{0} v_{0} \int_{0}^{V_{C d}} n(v) d v
$$

A definição convencional para o fluxo de nêutrons térmicos, $\phi_{t}$, é dada por $v_{0} \int_{0}^{V_{C d}} n(v) d v$. Assim:

$$
\int_{0}^{V_{C d}} \sigma(v) \phi(v) d v=\sigma_{0} \phi_{t}
$$

E a segunda integral é expressa como:

$$
\int_{V_{C d}}^{\infty} \sigma(v) \phi(v) d v=\int_{E_{C d}}^{\infty} \sigma(E) \phi(E) d E
$$

O fluxo de nêutrons epitérmicos, como observado na EQ. (2.7), não obedece exatamente a proporcionalidade $\phi_{e}(E) \sim \frac{1}{E}$. É necessário inserir o parâmetro de correção $\alpha$.

Para energias acima de $0.35 \mathrm{eV}$, o fluxo de nêutrons pode ser definido como: 


$$
\phi(E)=\frac{\phi\left(E_{r e f}\right)}{\left(\frac{E}{E_{\text {ref }}}\right)^{1+\alpha}}
$$

A energia de referência $E_{\text {ref }}$ é um parâmetro escolhido arbitrariamente. Sua integral de ressonância é expressa como:

$$
I(\alpha)=\int_{E_{C d}}^{\infty} \sigma(E) \frac{E_{r e f}^{\alpha}}{E^{1+\alpha}} d E
$$

e,

$$
\phi_{e}=\phi\left(E_{r e f}\right) E_{r e f}
$$

Assim:

$$
\int_{E_{C d}}^{\infty} \sigma(E) \phi(E) d E=\phi_{e} I_{0}(\alpha)
$$

e a taxa de reação $R$ é dada por:

$$
R=\sigma_{0} \phi_{t}+\phi_{e} I_{0}(\alpha)
$$

sendo, portanto, a taxa de reação como a soma das contribuições dos nêutrons térmicos e epitérmicos.

Além das convenções de Høgdahl e de Westcott, que são bastante utilizadas e avaliadas na área da ANN, outras convenções também são conhecidas, como a convenção de Menno Blaauwi ${ }^{[4]}$ e a convenção de Stoughton-Halperin ${ }^{[46]}$.

\subsection{Análise de Ativação Neutrônica e o Método $\boldsymbol{k}_{0}$}

A AAN é uma técnica utilizada para determinar as concentrações de elementos em uma dada amostra, sendo capaz de analisar várias simultaneamente. A amostra pode ser líquida (aquosa ou orgânica) ou sólida (animal, tecido, cerâmica, vidros, papeis, plásticos, metais etc.) ${ }^{[47]}$. 
A forma mais comum de AAN é a Análise de Ativação Neutrônica Instrumental (AANI). Nesta técnica, uma quantidade de amostras com suas massas medidas são encapsuladas adequadamente para serem analisadas e enviadas pneumaticamente (ou manualmente) em um local de irradiação de um reator nuclear de pesquisa, sendo bombardeada por nêutrons que são produzidos no reator. Uma vez irradiada em um determinado tempo, a amostra é retirada do local e a atividade induzida é medida pela contagem em um espectrômetro de raios-gama. O resultado da contagem produz um espectro no qual específicas energias de raios-gama produzem picos, cuja áreas são proporcionais à quantidade do elemento que é responsável pelos raios-gama. Para determinar a quantidade real de um elemento em uma amostra, as áreas dos picos são comparadas com informações da literatura, ou ainda, a partir de um elemento utilizado como referência[47].

Existem três tipos de reações de ativação por nêutrons: reação $(\mathrm{n}, \gamma)$, reação $(n, p)$ e reação $(n, \alpha)$. A primeira reação é gerada por nêutrons térmicos, enquanto as demais são geradas, principalmente, por nêutrons epitérmicos e rápidos, respectivamente. A reação nuclear induzida por nêutrons, de grande importância para a AAN, é a de captura de nêutrons $(n, \gamma)$. Neste processo o núcleo composto libera a energia de excitação por meio da emissão de radiação eletromagnética.

A AAN é um método de análise não destrutivo que é aplicável em quase todo campo de interesse científico ${ }^{[48-56]}$. É capaz de determinar concentrações na ordem de partes por bilhão ou superior. No passado eram utilizados detectores de cintilação de $\mathrm{Nal}(\mathrm{TI})$ para a medida e identificação de raios-gama, entretanto, apresentavam baixa resolução em energia, o que em razão do grande número de radionuclídeos gerados tornava-se necessária a separação radioquímica dos elementos para eliminar a superposição dos picos do espectro $^{[57]}$. Com o advento dos detectores semicondutores de germânio de alta resolução, aliado ao uso dos analisadores multicanais, houve um avanço significativo na determinação de muitos elementos, eliminando em grande parte a necessidade e as incertezas ocasionadas pelo tratamento radioquímico. Atualmente, estão disponíveis programas sofisticados para análise espectral, que são ferramentas poderosas para a área de ativação neutrônica[58]. A necessidade de um reator nuclear como fonte de nêutrons pode ser uma desvantagem para a 
AAN em vista da sua limitação de utilização e pesquisa, sendo aplicável somente em setores que possuem um reator, além da instrumentação para medir a radioatividade gama.

Os avanços alcançados dentro da técnica de AAN ao longo do tempo foram importantes para ampliar o número de elementos analisados, gerando resultados com maior precisão e exatidão. $O$ método baseado em um único comparador tem sido amplamente avaliado e demonstrou-se ser tão eficiente (ou mais) quanto o método relativo ${ }^{[59]}$. O termo "único comparador" refere-se à técnica cuja análise multi-elementar é realizada por meio da irradiação e medição utilizando um único elemento comparador. A taxa de contagem específica da amostra, $A_{s p}$, pode ser calculada pela multiplicação da taxa de contagem específica do comparador, $A_{s p, c}$, com o fator comparador experimentalmente determinado, $k$, expresso como:[60]

$$
k=\frac{A_{s p}}{A_{s p, c}}=\frac{M_{c}}{M} \frac{\gamma}{\gamma_{c}} \frac{\varepsilon_{p}}{\varepsilon_{p, c}} \frac{\theta}{\theta_{c}} \frac{\widehat{\Phi}}{\widehat{\Phi}_{c}} \frac{\widehat{\sigma}}{\widehat{\sigma}_{c}}
$$

sendo:

$$
A_{s p}=\frac{N_{A} \theta \gamma}{M}\left(G_{t h} \widehat{\Phi}_{t h} \hat{\sigma}+G_{e} \widehat{\Phi}_{e} I_{0}(\alpha)\right) \varepsilon_{p}=\frac{N_{p / t_{m}}}{S D C w}
$$

onde:

$M$ = Massa atômica do elemento irradiado;

$\theta=$ Abundância isotópica do nuclídeo alvo;

$\gamma=$ Intensidade absoluta do raio-gama considerado;

$\varepsilon_{p}=$ Eficiência do detector para o pico de absorção total de energia do raio-gama considerado;

$\widehat{\Phi}=$ Fluxo de nêutrons do reator convencional (Fluxo total de Westcott) ${ }^{[34]}$;

$\widehat{\Phi}_{t h}=$ Fluxo de nêutrons térmicos convencional;

$\widehat{\Phi}_{e}=$ Fluxo de nêutrons epitérmicos convencional;

$\hat{\sigma}=$ Seção de choque efetiva;

$S=$ Fator de saturação dependente da constante de decaimento $\lambda$ e tempo de irradiação $t_{i r r}$, dado por: 


$$
1-e^{-\lambda t_{i r r}}
$$

$D=$ Fator de correção para o decaimento entre o início da medida e a data de referência;

$C=$ Fator de correção para o decaimento durante o tempo de medida;

$N_{p}=$ Área sob o pico de absorção total de energia;

$t_{m}=$ Tempo de medida;

$w=$ Massa do elemento.

O método do comparador acima descrito foi avaliado criticamente por GIRARDI et al.[60,61]. Novas aproximações foram desenvolvidas posteriormente como alternativas, utilizando outras convenções como de Høgdahl e StoughtonHalperin para o cálculo da taxa de reação $R$, introduzindo assim o fator $k_{0}{ }^{[60,62]}$, cujo diferencial era a não dependência do espectro de nêutrons na posição de irradiação e das características do detector utilizado, ou seja, o fator não incluía a secção de choque efetiva e a eficiência de detecção. O conceito do método $k_{0}$ pode ser aproximado por duas diferentes maneiras:[11]

Maneira 1: Se os fatores $k$ determinados experimentalmente pelo método do comparador único forem normalizados para as condições experimentais de irradiação $(f, \alpha)$ e contagens $(\varepsilon), k_{0}$ pode ser obtido a partir da equação:

$$
k_{0}(i)=k_{c}(i) \frac{G_{t h, c} f+G_{e, c} Q_{o, c}(\alpha) \varepsilon_{p, c}}{G_{t h, i} f+G_{e, i} Q_{o, i}(\alpha) \varepsilon_{p, i}}
$$

sendo $k_{c}(i)$ expresso por:

$$
k_{c}(i)=\frac{A_{s p, i}}{A_{s p, c}}=\frac{M_{c}}{M_{i}} \frac{\gamma_{i}}{\gamma_{c}} \frac{\theta_{\mathrm{i}}}{\theta_{c}} \frac{\sigma_{0, i}}{\sigma_{0, c}} \frac{G_{t h, i} f+G_{e, i} Q_{o, i}(\alpha) \varepsilon_{p, i}}{G_{t h, s} f+G_{e, c} Q_{o, c}(\alpha) \varepsilon_{p, c}}
$$

O fator $k_{0}$ é definido, portanto, como uma constante nuclear composta:

$$
k_{0, c}(i)=\frac{M_{c}}{M_{i}} \frac{\gamma_{i}}{\gamma_{c}} \frac{\theta_{\mathrm{i}}}{\theta_{c}} \frac{\sigma_{0, i}}{\sigma_{0, c}}
$$


Supondo que após a determinação dos valores de $k_{0}(i)=k_{0}(a)$ a amostra é co-irradiada com um monitor $(m)$ para qual possui um valor de $k_{0, c}(\mathrm{~m})$ experimentalmente determinado, a concentração do analito $c_{a}$ é obtida pela relação:

$$
c_{a, p p m}=\frac{A_{s p, a}}{A_{s p, m}} \frac{k_{o, c}(m)}{k_{0, c}(a)} \frac{G_{t h, m} . f+G_{e, m} Q_{o, m}(\alpha) \cdot \varepsilon_{p, m}}{G_{t h, a} . f+G_{e, a} Q_{o, a}(\alpha) \cdot \varepsilon_{p, a}} \cdot 10^{6}
$$

na condição em que não há variação isotópica entre o analito e a amostra $\left(\Theta_{a}=\right.$ $\left.\Theta_{i}\right)$.

Maneira 2: No método absoluto, a concentração é expressa por:

$$
c_{a, p p m}=\frac{A_{s p, a}}{A_{s p, m}} \frac{M_{a}}{M_{m}} \frac{\gamma_{m}}{\gamma_{a}} \frac{\Theta_{m}}{\Theta_{a}} \frac{\widehat{\sigma}_{m}}{\hat{\sigma}_{a}} \frac{G_{t h, m} . f+G_{e, m} Q_{o, m}(\alpha) \cdot \varepsilon_{p, m}}{G_{t h, a} f+G_{e, a} Q_{o, a}(\alpha) \cdot \varepsilon_{p, a}} \cdot 10^{6}
$$

Substituindo os dados nucleares $M_{m} \gamma_{a} \Theta_{a} \hat{\sigma}_{a} / M_{a} \gamma_{m} \Theta_{m} \hat{\sigma}_{m}$ por uma única constante nuclear, $k_{0, m}$, definida como o fator para o elemento analisado com referência ao monitor, a concentração do analito é obtida pela expressão:

$$
c_{a, p p m}=\frac{A_{s p, a}}{A_{s p, m}} \frac{1}{k_{0, m}(a)} \frac{G_{t h, m} . f+G_{e, m} Q_{o, m}(\alpha) \cdot \varepsilon_{p, m}}{G_{t h, a} . f+G_{e, a} Q_{o, a}(\alpha) \cdot \varepsilon_{p, a}} \cdot 10^{6}
$$

O método $k_{0}$ pode ser, portanto, interpretado como uma substituição dos dados nucleares pelo fator $k_{0}$ calculado experimentalmente. Isso elimina incertezas provenientes destes dados, tornando o resultado mais preciso, sob a condição, evidentemente, de que a determinação experimental de $k_{0}$ seja realizada corretamente.

O valor de $k_{0}$ também pode ser obtido por outro método além do demonstrado pela EQ. (2.38), por meio da técnica de subtração cádmica, expresso por:

$$
k_{0, c}(i)=\frac{A_{s p, i}-\frac{\left(A_{s p, i}\right) C d}{F_{C d, i}}}{A_{s p . c}-\frac{\left(A_{s p, c}\right)}{F_{C d, c}}} \cdot \frac{G_{t h, c .} \varepsilon_{p, c}}{G_{t h, i .} \varepsilon_{p, i}}
$$


com o diferencial de não necessitar da introdução de $f$ e $Q_{0}(\alpha)$.

O comparador usado por conveniência para manter os dados de $k_{0}$ condensados e organizados é o ouro $\left({ }^{198} \mathrm{Au}\right)$, entretanto, outros monitores podem ser utilizados, como Co, Zn, Zr etc., bastando fazer a conversão dada por (o índice $A u$ representa o comparador $c)^{[39] \text { : }}$

$$
k_{0, m}(i)=\frac{k_{0, A u}(i)}{k_{0, A u}(m)}
$$

Para medidas de $k_{0}$ precisas em aplicações práticas, dois parâmetros do espectro do reator, $f$ e $\alpha$, devem ser analisados, e dois parâmetros nucleares $Q_{0}$ e $\bar{E}_{r}$, e a curva de eficiência em energia do detector também devem ser conhecidos. O uso combinado de ${ }^{198} \mathrm{Au},{ }^{94} \mathrm{Zr}$ e ${ }^{96} \mathrm{Zr}$ como monitores se tornou mais conveniente em razão da baixa contribuição às incertezas para o cálculo de $f$ e $\alpha$ e pelas características de ambos Zr citados na sessão 2.4. Se a irradiação com cádmio, por algum motivo, não for possível ou se a condição de constância de $f$ não pode ser validado ou provada, pode-se calcular $\alpha$ e $f$ sem a necessidade do uso de uma cobertura de cádmio, utilizando um isótopo, usualmente ${ }^{94} \mathrm{Zr}$ ou ${ }^{198} \mathrm{Au}$ como um comparador, enquanto os pares ${ }^{94} \mathrm{Zr}-{ }^{96} \mathrm{Zr}$ e ${ }^{198} \mathrm{Au}-{ }^{96} \mathrm{Zr}$ são usados como monitores de fluxo independentes. As razões de fluxo obtidas serão iguais se $\alpha=0$ ou se os valores $Q_{0}$ utilizados forem corrigidos para $\alpha$ durante os cálculos. $\alpha$ e $f$ podem então ser simultaneamente determinados sem o uso da cobertura cádmica ${ }^{[39,63]}$.

\subsection{Determinação de $\alpha$ para o fluxo de nêutrons epitérmicos}

O comportamento do fluxo de nêutrons epitérmicos em uma situação real é representado por uma aproximação do tipo $1 / E^{1+\alpha}$, como expresso na $\mathrm{EQ}$. (2.7) e EQ. (2.8), e na FIG. 1. O parâmetro $\alpha$, como dito, deve ser determinado experimentalmente. As metodologias para a sua determinação, de acordo com De Corte ${ }^{[11]}$, podem ser classificadas em três grupos, que correspondem a uma medida de razão de cádmio (Cd-ratio measurements), irradiação coberta com cádmio (Cd-covered irradiation) e irradiação descoberta (bare irradiation). 


\subsubsection{Método da razão de cádmio com monitores múltiplos}

Um conjunto de monitores é irradiado com e sem cobertura de cádmio. A atividade induzida é medida em um detector de germânio hiperpuro (HPGe). Se considerar que todos os monitores possuem uma dependência que tendem à lei$1 / v$ para energias de até $1,5 \mathrm{eV}, \alpha$ pode ser obtido pela inclinação da curva ao desenhar o gráfico da expressão (o índice $i$ refere-se a cada núcleo alvo):

$$
\ln \frac{\left(\bar{E}_{r, i}\right)^{-\alpha}}{\left(F_{C d, i} \cdot R_{C d, i}-1\right) \cdot Q_{0, i}(\alpha) \cdot \frac{G_{e, i}}{G_{t h, i}}} \text { versus } \ln \bar{E}_{r, i}
$$

onde:

$\bar{E}_{r, i}=$ Energia de ressonância média;

$F_{C d, i}=$ Fator de transmissão de cádmio;

$R_{C d, i}=$ Razão entre a taxa de contagem específica do isótopo irradiado sem cobertura de Cádmio e a taxa de contagem específica do isótopo irradiado com cobertura de Cádmio;

$Q_{0, i}(\alpha)=$ Razão entre a integral de ressonância e a seção de choque térmica do núcleo alvo em função de $\alpha$;

$G_{e, i}=$ Correção para auto blindagem de nêutrons epitérmicos.

De Corte ${ }^{[11]}$ sugere, para este método, evitar a utilização de monitores com alto valor de $Q_{0}$ em virtude do aumento de fatores de propagação de erros, especialmente em locais de irradiação com baixo fluxo. Também enfatiza evitar a termalização por espaçadores de polietileno nos recipientes para as irradiações com e sem cádmio. Por outro lado, desde que somente razões de cádmio estejam envolvidas, qualquer geometria de contagem pode ser utilizada.

\subsubsection{Método de monitores múltiplos cobertos com cádmio}

De modo semelhante ao método anterior, um conjunto de monitores é irradiado simultaneamente sob uma cobertura de cádmio e a atividade é medida por meio de um detector de germânio hiper puro (HPGe) com a eficiência de detecção conhecida. Se as seções de choque dos monitores aproximam-se da lei- 
$1 / v$ para energias de até $1,5 \mathrm{eV}$, o parâmetro $\alpha$ pode ser obtido pela inclinação da curva ao graficar ${ }^{[9,64]}$ :

$$
\ln \frac{\left(\bar{E}_{r, i}\right)^{-\alpha}\left(A_{s p, i}\right)_{C d}}{k_{0, c}(i) \varepsilon_{p, i} F_{C d, i} Q_{0, i}(\alpha) G_{e, i}} \text { versus } \ln \bar{E}_{r, i}
$$

onde:

$\left(A_{s p, i}\right)_{C d}=$ Taxa de contagem específica (Área do pico de absorção total de energia para a reação), corrigida pelo fator de saturação, tempo de decaimento, soma em cascata, geometria, tempo de medida e massa;

$k_{0, c}(i)=$ Fator $k_{0}$ do monitor com relação ao comparador (usualmente $\mathrm{Au}$ );

$\varepsilon_{p, i}=$ Eficiência de pico do detector HPGe para a energia gama do monitor;

O índice $c$ refere-se ao elemento comparador. Como o lado esquerdo da EQ. (2.47) também depende do parâmetro $\alpha$, um procedimento iterativo tornase necessário para obter um valor final, começando por plotar a EQ. (2.47) com $\alpha=0$ que oferece a primeira aproximação de $\alpha=\alpha_{1}$ e assim por diante. $\mathrm{O}$ uso de três monitores com $\bar{E}_{r, i}$ bem afastados entre si podem trazer resultados com maior precisão e exatidão para o monitoramento de $\alpha^{[11,64]}$.

\subsubsection{Método Múltiplos Monitores descobertos}

Este método consiste em irradiar um conjunto de monitores, juntos com um monitor de referência, sem a cobertura de cádmio, e a atividade induzida é medida por meio de um detector de HPGe com uma eficiência de detecção conhecida. Assim como os métodos anteriores, se as seções de choque dos monitores tendem à lei-1/v para energias de até 1,5 eV, o parâmetro $\alpha$ pode ser obtido pela inclinação da curva ao desenhar o gráfico:

$$
\ln \left\{\left(\bar{E}_{r, i}\right)^{-\alpha} \cdot \frac{\frac{A_{s p, i}}{k_{0, A u}(i)} \varepsilon_{p, i}-\frac{A_{s p, r e f}}{k_{0, A u}(r e f)} \varepsilon_{p, r e f}}{\frac{Q_{0, i(\alpha)} G_{e, i}}{G_{t h, i}}-\frac{Q_{o, r e f(\alpha)^{G}, r e f}}{G_{t h, r e f}}}\right\} \text { versus } \ln \bar{E}_{r, i}
$$


O índice ref está associado ao monitor de referência. O número mínimo de monitores é 3, sendo dois monitores mais um de referência. Esta configuração é uma técnica conhecida como Método dos Três Monitores Descobertos (BareTriple Monitor Method).

Os fatores de propagação de erros e as incertezas resultantes que envolvem $\alpha$ são, em geral, maiores neste método em comparação com os métodos anteriormente citados ${ }^{[11]}$.

\subsection{Determinação do parâmetro $Q_{0}$ e o Fator de Cádmio $F_{C d}$}

Os valores de $Q_{0}$ são definidos como a razão entre a integral de ressonância $I_{0}$ em um espectro do tipo $1 / E$ pela seção de choque térmica $\sigma_{o}$, correspondente a um nêutron com velocidade de $2200 \mathrm{~m} / \mathrm{s}^{[40,65]}$ :

$$
Q_{0}=\frac{I_{0}}{\sigma_{0}}
$$

A integral de ressonância, como representado na EQ. (2.6), pode ser definido pelo método da razão de cádmio, onde $t(E)$ é uma função de transmissão cádmica:

$$
I_{C d}=\int_{0}^{\infty} t(E) \sigma(E) \phi^{*}(E) d E
$$

Onde $\phi^{*}(E)$ refere-se ao espectro com perturbações. A EQ. (2.49) reduz-se para EQ. (2.6) se definir os limites da integral entre as energias de corte de cádmio $E_{C d}$ até $E_{3}$, que corresponde a $2 \mathrm{MeV}$. Os efeitos de auto blindagem devem ser desprezíveis de modo que o espectro $\phi^{*}(E)$ seja igual ao espectro sem perturbações $\phi(E)^{[65]}$, ou seja, que obedece a forma $1 / E$, e $t(E)$ é uma função degrau idealizada do tipo:

$$
t(E)=\begin{aligned}
& 0 \text { se } E<E_{C d} \\
& 1 \text { se } E \geq E_{C d}
\end{aligned}
$$

O termo $t(E)$ pode ser expresso de um modo mais realístico, como: 


$$
t(E)=e^{-N_{C d} d \sigma_{C d}(E)}
$$

onde:

$d=$ Espessura da cobertura de cádmio;

$\sigma_{C d}=$ Seção de choque total do cádmio;

$N_{C d}=$ Número de átomos da cobertura de cádmio, dado por:

$$
N_{C d}=\frac{\rho_{C d} N_{A}}{M_{C d}}
$$

onde:

$\rho_{C d}=$ Densidade do cádmio;

$N_{A}=$ Número de Avogadro;

$M_{C d}=$ Massa molar do cadmio

A integral de ressonância da EQ. (2.49) é uma medida a ser comparada com a EQ (2.6) nos limites da energia de corte de cádmio. Introduz-se um fator de cádmio $F_{C d}$ para corrigir eventuais diferenças ${ }^{[65]}$ :

$$
I=\int_{E_{C d}}^{E_{3}} \sigma(E) \phi(E) d E=\frac{1}{F_{C d}} \int_{0}^{\infty} t(E) \sigma(E) \phi(E) d E
$$

Assim:

$$
F_{C d}=\frac{\int_{0}^{\infty} t(E) \sigma(E) \phi(E) d E}{\int_{E_{C d}}^{E_{3}} \sigma(E) \phi(E) d E}
$$

Os desvios de $F_{C d}$ de uma unidade podem ser por fatores relacionados com a função de transmissão cádmica.

A determinação do valor de $Q_{0}$ é possível de ser realizada por meio da medida da razão de cádmio, expresso por:[11, 63]:

$$
Q_{0}=\frac{F_{C d, c} R_{C d, c}-1}{F_{c d, i} R_{C d, i}-1} \frac{G_{t h, i}}{G_{t h, c}} \frac{G_{e, c}}{G_{e, i}} Q_{o, c}
$$


onde $R_{C d}$ é a razão de cádmio. A razão de cádmio é definida como sendo a razão entre as taxas de contagens específicas da amostra com e sem cobertura de cádmio, dada por:

$$
R_{C d, i}=\frac{A_{s p, i}}{\left(A_{s p}\right)_{C d, i}}
$$

Sendo $A_{s p, i}$ e $\left(A_{s p}\right)_{c d, i}$ as taxas de contagens específicas da amostra sem cádmio e com cádmio, respectivamente. Aplicando o fator $\alpha$ de aproximação na $\mathrm{EQ}$. (2.55), considerando as EQ; (2.32) e (2.49), chega-se, portanto, à expressão:

$$
Q_{0}(\alpha)=\frac{I_{0}(\alpha)}{\sigma_{0}}
$$

Assim, pelo método da Razão de Cádmio, define-se $Q_{o}(\alpha)$ como:

$$
Q_{0}(\alpha)=\frac{F_{C d, c} R_{C d, c}-1}{F_{c d, i} R_{C d, i}-1} \frac{G_{t h, i}}{G_{t h, c}} \frac{G_{e, c}}{G_{e, i}} Q_{o, c}(\alpha)
$$

ou:

$$
Q_{0}(\alpha)=\frac{f}{F_{c d, i} R_{C d, i}-1} \frac{G_{t h, i}}{G_{e, i}}
$$

onde $f$ é a razão entre os fluxos térmicos e epitérmicos, que pode ser obtida também por meio do método da Razão de Cádmio:

$$
f=\frac{\varphi_{t h}}{\varphi_{e}}=\left(F_{C d, c} R_{c d, c}-1\right) G_{e, c} \frac{Q_{o, c}(\alpha)}{G_{t h, c .}}
$$

ou por meio do método dos Monitores Bi-Isotópicos Descobertos. Este método consiste em irradiar um conjunto de monitores sendo dois isótopos cuja características sejam bem conhecidas e com valores de $Q_{0}$ bastante afastados entre si[58], e um monitor de referência. $O$ parâmetro $f$ é calculado pela expressão:

$$
f=\frac{G_{e, i} \frac{k_{0, c}(1) \varepsilon_{p, 1}}{k_{0, c}(2) \varepsilon_{p, 2}} Q_{0,1}(\alpha)-G_{e, 2} \frac{A_{s p, 1}}{A_{s p, 2}} Q_{0,2}(\alpha)}{G_{t h, 2} \frac{A_{s p, 1}}{A_{s p, 2}} G_{t h, 1}-G_{t h, 1} \frac{k_{0, c}(1) \varepsilon_{p, 1}}{k_{0, c}(2) \varepsilon_{p, 2}}}
$$


Onde, usualmente, utiliza-se os isótopos ${ }^{95} \mathrm{Zr}$ e ${ }^{97} \mathrm{Zr}$, que melhor representam as características citadas, indicados pelos índices 1 e 2 na EQ. (2.62), respectivamente.

A relação entre $Q_{0}$ e $Q_{0}(\alpha)$ é dada por ${ }^{[11]}$ :

$$
Q_{0, i}(\alpha)=\frac{Q_{0 i}-0,429}{E_{i}^{\alpha}}+\frac{0,429}{(2 \alpha+1) \cdot 0,55^{\alpha}}
$$

\subsection{Outros parâmetros que compõem o método $\boldsymbol{k}_{0}$}

\subsubsection{Eficiência de detecção}

Todos os detectores de radiação irão, em princípio, dar origem a um pulso de saída para cada quantum de radiação que interaja dentro do seu volume ativo. Para radiações de partículas carregadas tais como alfa ou beta, a interação, sob a forma de ionização ou excitação, ocorrerá imediatamente após a entrada da partícula no volume. Uma partícula formará pares de íons ao longo de seu caminho garantindo que o pulso resultante é grande o suficiente para ser registrado. Desse modo, um detector irá detectar cada partícula alfa ou beta que entra em seu volume ativo. Nestas condições, diz-se que o detector tem uma eficiência de contagem de $100 \%[22,66]$.

As radiações não carregadas, como radiações gama ou nêutrons, devem primeiro sofrer uma interação significativa antes da detecção ser possível. Como essas radiações podem percorrer grandes distâncias entre as interações, ocorre a condição dos detectores não serem 100\% eficientes. Torna-se necessário ter uma correção precisa para a eficiência do detector, a fim de relacionar o número de pulsos contados para o número de nêutrons ou fótons incidentes sobre o detector. É conveniente subdividir as eficiências de contagem em duas classes: absoluta e intrínseca. As eficiências absolutas são definidas como:

$$
\varepsilon_{a b s}=\frac{\text { número de pulsos registrados }}{\text { número de fótons de radiação emitidos pela fonte }}
$$


e não dependem somente das propriedades do detector, mas também da geometria de detecção, como a distância entre a fonte e o detector. A eficiência intrínseca é definida como:

$$
\varepsilon_{\text {int }}=\frac{\text { número de pulsos registrados }}{\text { número de fótons de radiação incidentes no detector }}
$$

e dependende do material do detector, da energia da radiação e da espessura física do detector na direção em que a radiação está incidindo.

Em medidas de atividade de uma fonte radioativa ou da probabilidade de emissão gama por decaimento de um radionuclídeo, um dos parâmetros mais importantes é a eficiência de detecção no intervalo de energia de interesse e na geometria do arranjo experimental. Neste caso, a eficiência é denominada eficiência de pico, $\varepsilon_{p}$. A eficiência de pico supõe que somente as interações que depositarem toda a energia da radiação incidente serão registradas e corresponde à razão entre o número de eventos registrados no pico de absorção total e o número de fótons emitidos pela fonte, sendo representada pela relação:

$$
\varepsilon_{p}(E)=\frac{N_{p}}{\gamma A t} f(E)
$$

onde:

$A=$ Atividade da fonte;

$t=$ Tempo de medida;

$f(E)=$ Constituído por todos os fatores de correções que devem ser aplicados, tais como: decaimento radioativo, atenuação na fonte, tempo morto, soma em cascata, atenuação da fonte e geometria de detecção. Nota-se que estes fatores dependem da energia considerada ${ }^{[18]}$.

A determinação de $\varepsilon_{p}$ é realizada pela medição da eficiência em condições de referência usando fontes calibradas, ou seja, padronizadas em atividade. As fontes padronizadas normalmente têm uma geometria ligeiramente diferente das amostras a serem analisadas, necessitando aplicar uma correção para a posição da fonte padrão. Podem ocorrer detecções simultâneas de outras 
radiações gama, que surgem em cascata com o gama a ser medido. Se estas radiações em cascata forem detectadas durante um intervalo de tempo menor que o tempo de resolução do espectrômetro, o pulso resultante corresponderá à soma dos pulsos individuais. Desta forma, o número de contagens sob o pico de absorção total será alterado. Para corrigir este problema podem ser utilizadas simulações baseadas no método de Monte Carlo[67].

\subsubsection{Correções de auto-blindagem}

Para materiais contendo nuclídeos com grandes seções de choque é importante considerar o fatores de auto-blindagem para nêutrons térmicos e epitérmicos (self-shielding factors). Amostras biológicas, por exemplo, costumam possuir uma certa vantagem para a o método AAN em razão de não conterem elementos com altos níveis de seção de choque para reações $(n, \gamma)$ e, portanto, os efeitos de auto-blindagem para nêutrons térmicos são pequenos ${ }^{[1]}$

A presença de uma amostra num campo de nêutrons térmicos ou epitérmicos de um reator nuclear cria uma perturbação no fluxo de nêutrons no local. Este efeito pode ser de considerável importância se a seção de choque da amostra exibir um relevante pico de ressonância para o campo de nêutrons considerado. Em uma situação típica, a atividade $A$ de um dado nuclídeo produzido pela reação $(n, \gamma)$ por meio da irradiação de uma amostra contendo uma quantidade $m$ de elemento é dada por:

$$
A=\frac{m N_{A} \theta}{M} \sigma_{t h} \varphi_{\mathrm{th}}\left(G_{t h}+\frac{G_{e} Q_{0}}{f}\right)\left(1-e^{-\lambda t_{i r r}}\right)
$$

sendo $f=\varphi_{t h} / \varphi_{e p}$, conforme definido na EQ. (2.61), $\varphi_{t h}$ e $\varphi_{e p}$, são fluxos térmicos e epitérmicos não perturbados, respectivamente. Na prática, $\varphi_{t h}$ e $\varphi_{e p}$ são medidos através da irradiação simultânea de monitores de fluxo próximo da amostra absorvedora. Para irradiações curtas e assumindo que $\varphi_{t h}$ e $\varphi_{e p}$ são constantes com o tempo, pode-se medi-los irradiando monitores de fluxo antes ou depois da amostra, na mesma posição. O uso de simulações com método de Monte Carlo também auxilia na obtenção dos valores de $G_{t h}$ e $G_{e p}$, por meio de 
uma simulação comparando a amostra real com uma amostra infinitamente diluida ${ }^{[65,66]}$.

Os fatores de correção $G_{t h}$ e $G_{e p}$ dependem da geometria da amostra e são iguais a uma unidade quando as amostras estão suficientemente diluídas. $O$ fator $G_{t h}$ depende da quantidade dos elementos na amostra que absorvem nêutrons térmicos, enquanto $G_{e p}$ pode ser influenciado por outros nuclídeos, como isótopos do mesmo elemento ou isótopos de elementos diferentes presentes na amostra, contudo, somente se esses nuclídeos absorverem nêutrons com energias de ressonância que se sobrepõem com as ressonâncias que ativam o nuclídeo em questão.

Uma função sigmóide universal foi desenvolvida para os cálculos de $G_{t h}$ e $G_{e p}{ }^{[69,70]}$. Para nêutrons térmicos a expressão é dada por:

$$
G_{t h}=\frac{1}{1+\left(\frac{z}{1,029}\right)^{1,009}}
$$

onde:

$$
z=x \Sigma_{t}\left(\frac{\Sigma_{a}}{\Sigma_{\mathrm{t}}}\right)^{k}
$$

sendo:

$k=0,85 \pm 0,05$;

$\Sigma_{t}=$ Seção de choque macroscópica total;

$\Sigma_{a}=$ Seção de choque macroscópica de absorção;

$x=$ Espessura ou raio, para o caso da amostra ser uma folha, um fio ou esfera.

Para nêutrons epitérmicos a expressão é dada por:

$$
G_{e}=\frac{0,94}{1+\left(\frac{z}{2,70}\right)^{0,82}}+0,06
$$

onde:

$$
z=\Sigma_{t}\left(E_{r e s}\right) y\left(\frac{\Gamma_{\gamma}}{\Gamma}\right)^{\frac{1}{2}}
$$

sendo:

$y=\mathrm{O}$ raio $R$ para amostra esférica ou $2 R$ para amostra cilíndrica; a espessura $t$, sendo 1,5t para folhas; 
$\Gamma=$ Largura total de nível de excitação do núcleo-alvo;

$\Gamma_{\gamma}=$ Largura de nível para o canal de saída gama;

$\frac{\Gamma_{\gamma}}{\Gamma}=$ Probabilidade de decaimento para o canal de saída gama.

A estimativa de $G_{e p}$ como função da concentração do elemento a da geometria da amostra é uma tarefa complexa, em razão de cada nuclídeo possuir um padrão de ressonância diferente e às vezes complexo[71]. O uso do método de Monte Carlo pode ser dispendioso e não muito prático para situações de rotina de AAN. Uma maneira comum tem sido evitar este problema diluindo as amostras ou irradiando em um espectro de nêutrons com baixo componente epitérmico.

\subsection{Análises das incertezas}

Dados analisados usualmente são processados através de multiplicação, adição ou outra manipulação funcional para chegar a um resultado de interesse. Os valores que são produzidos por estas etapas são distribuídos de uma forma que é dependente tanto da distribuição original como dos tipos de operações realizadas. Embora o valor médio da distribuição proveniente seja simplesmente calculado a partir das médias das distribuições originais, os valores do desvio padrão não são tão óbvios. Pode-se mostrar que se os erros são individualmente pequenas e simétricas em torno de zero, é possível obter um resultado geral para que a incerteza esperada seja associada a qualquer quantidade que é calculada como uma função das variáveis independentes. Se $x$, $y, z, \ldots$ são contagens diretamente medidas ou outras variáveis relacionadas para as quais são conhecidas $\sigma_{x}, \sigma_{y}, \sigma_{z}, \ldots$ então o desvio padrão para qualquer quantidade $u$ pode ser calculado a partir da expressão[22]:

$$
\sigma_{u}^{2}=\left(\frac{\delta u}{\delta x}\right)^{2} \sigma_{x}^{2}+\left(\frac{\delta u}{\delta y}\right)^{2} \sigma_{y}^{2}+\left(\frac{\delta u}{\delta z}\right)^{2} \sigma_{z}^{2}+\cdots
$$

onde $u=(x, y, z, \ldots)$ representa a quantidade derivada. A equação (2.72) é usualmente conhecida como a fórmula de propagação de erros (incertezas) e é aplicável a quase todas as situações em medições nucleares. As variáveis $x, y$, 
$z, \ldots$, se forem verdadeiramente independentes, não apresentarão efeitos de correlação.

É possível, também, analisar as incertezas por meio de uma matriz de covariância, uma forma compacta e otimizada de escrever as variâncias e as covariâncias de um dado. Considerando $n$ grandezas físicas $y_{1}, y_{2} \ldots y_{n}$, a matriz de covariância é definida por ${ }^{[20]}$

$$
V_{y}=\left(\begin{array}{cccc}
\sigma_{1}^{2} & \operatorname{COV}\left(y_{1} y_{2}\right) & \cdots & \operatorname{COV}\left(y_{1} y_{n}\right) \\
\operatorname{COV}\left(y_{1} y_{2}\right) & \sigma_{2}^{2} & \cdots & \operatorname{COV}\left(y_{2} y_{n}\right) \\
\vdots & \vdots & \ddots & \vdots \\
\operatorname{COV}\left(y_{1} y_{n}\right) & \operatorname{COV}\left(y_{2} y_{n}\right) & \cdots & \sigma_{n}^{2}
\end{array}\right)
$$

Os termos diagonais são as variâncias das respectivas grandezas e os termos fora da diagonal são as covariâncias entre elas. Por ser uma matriz simétrica, $\operatorname{COV}\left(y_{i} y_{j}\right)=\operatorname{COV}\left(y_{j} y_{i}\right)$. A covariância é calculada como[20, 72]:

$$
\sigma_{x y}=\frac{1}{N-1} \Sigma\left(x_{i}-\bar{x}\right)\left(y_{i}-\bar{y}\right)
$$

onde $N$ é um número de pares de medidas $\left(x_{1}, y_{1}\right), \ldots,\left(X_{N}, y_{N}\right)$ de duas variáveis $x$ e $y$ e $\bar{x}$ e $\bar{y}$ são os valores esperados das mesmas, respectivamente.

O coeficiente de correlação, cuja função verifica quão correlacionadas estão duas grandezas, é dado por ${ }^{[72]}$ :

$$
r=\frac{\sigma_{x y}}{\sigma_{x} \sigma_{y}}=\frac{\Sigma\left(x_{i}-\bar{x}\right)\left(y_{i}-\bar{y}\right)}{\sqrt{\Sigma\left(x_{i}-\bar{x}\right)^{2} \Sigma\left(y_{i}-\bar{y}\right)^{2}}}
$$

A metodologia que utiliza as covariâncias é importante para que se possa obter resultados com o valor mais rigoroso da incerteza na grandeza mensurada. As referências da literatura possuem alguns trabalhos que procuraram desenvolver metodologias para este fim, determinando concentrações dos elementos químicos pelo método $k_{0}{ }^{[73-75]}$. Porém muitos trabalhos verificados não consideraram as dependências dos parâmetros, calculando as possíveis correlações existentes entre si, tratando-os como variáveis independentes ou 
mesmo não explicitando tal questão. Outros trabalhos usam os programas KAYZERO e $k_{0}$-IAEA para as análises ${ }^{[36,52,76,77,78]}$, contudo, verificando 0 manual, nota-se que algumas fontes de incertezas não estão incluídas nos cálculos, o que pode apresentar resultados com incertezas que não sejam totalmente satisfatórios ou até mesmo incompletos.

As teorias e equações definidas até o momento foram utilizadas para a construção e atualização dos códigos COVAR e AKFIT, resultando assim em um método para calcular os valores de $k_{0}$ e $Q_{0}$ e suas incertezas com, potencialmente, maior precisão, utilizando as matrizes de covariância. 


\section{METODOLOGIA}

\subsection{Atualização do código COVAR}

O desenvolvimento dos códigos presentes neste trabalho teve como base os procedimentos adotados a seguir. Os valores de $k_{0}$ de cada nuclídeo $i$ em relação ao comparador $c$ (para o presente trabalho foi utilizado ${ }^{198} \mathrm{Au}$ ) são determinados através de medições das atividades das amostras irradiadas com e sem cobertura de cádmio, como definido pela EQ. (2.44):

$$
k_{0, A u}(i)=\frac{A_{s p, i}-\frac{\left(A_{s p, i}\right) C d}{F_{C d, i}}}{A_{s p . A u}-\frac{\left(A_{s p, A u}\right) C d}{F_{C d, A u}}} \cdot \frac{G_{t h, A u .} \varepsilon_{p, A u}}{G_{t h, i .} \varepsilon_{p, i}}
$$

onde os índices $i$ e $\mathrm{A} u$ referem-se à amostra e ouro (comparador), respectivamente, e a taxa de contagem específica, $A_{s p}$, é definida pela EQ. (2.36).

Outra grandeza importante é a razão entre a integral de ressonância e a secção de choque para nêutrons térmicos, $Q_{0}$. Os valores de $Q_{0, i}(\alpha)$ dependem do parâmetro $\boldsymbol{\alpha}$ de acordo com a EQ. (2.63) e são obtidos pelos dados experimentais por meio da EQ. (2.56):

$$
Q_{0, i}(\alpha)=\frac{\left(F_{C d, A u} \cdot R_{C d, A u}-1\right) G_{t h, i} G_{e p, A u}}{\left(F_{C d, i} \cdot R_{C d, i}-1\right) G_{t h, A u} G_{e p, i}} Q_{0, A u}(\alpha)
$$

com a razão de cádmio, $R_{C d}$, dada pela EQ. (2.57).

O código COVAR original[18] utiliza as equações (3.1), (2.36), (2.63), (3.2) e (2.57) para as definições dos valores de $k_{0, A u}(i), Q_{0, i}(\alpha), Q_{0, i}, A_{s p, i,}\left(A_{s p}\right)_{C d, i}$ e $R_{C d, i}$ (aqui, o índice $\boldsymbol{i}$ refere-se tanto para a amostra quanto para o comparador, $\mathrm{Au})$.

Neste trabalho foi adicionado a expressão para o cálculo do $k_{0}$ que não utiliza os dados referentes às medições com cobertura de $C d$, dada pela $\mathrm{EQ}$. (2.38): 


$$
k_{0, A u}(i)=\frac{A_{s p, i}}{A_{s p . A u}} \cdot \frac{G_{t h, A u .} f+G_{e p, A u} Q_{0, A u}(\alpha) \varepsilon_{p, A u}}{G_{t h, i .} f+G_{e p, i} Q_{0, i}(\alpha) \varepsilon_{p, i}}
$$

Também foram corrigidas certas limitações possuídas pelo COVAR em sua versão atual até este presente trabalho (COVAR v3). Uma das limitações era o número máximo de alvos que podem ser analisados em um arquivo de entrada. Outra limitação era sobre a análise das correlações entre $k_{0}$ e $Q_{0}$, construídas para alguns casos específicos, e que foi generalizada para qualquer caso.

\subsection{Ajuste Único}

\subsubsection{Ajuste linear de $\alpha$ e $k_{0}$}

Um procedimento baseado no método dos mínimos quadrados ${ }^{[19]}$ foi desenvolvido para ajustar valores de $k_{0}$ de vários radionuclídeos que tiveram suas atividades determinadas por irradiação com e sem blindagem de cádmio. Neste procedimento, além de $k_{0}$, o valor de $\alpha$ também é determinado em um ajuste linear nos parâmetros. Para isso, a expressão que envolve $\alpha$ no cálculo do $k_{0}$ foi manipulada algebricamente para possibilitar seu cálculo por meio de um ajuste linear.

As EQ. (2.46) e (2.47), utilizadas para calcular $\alpha$ pela inclinação da curva, podem ser expressas como:

$$
Y_{i}=a+\alpha X_{i}
$$

onde:

$X_{i}=\ln \bar{E}_{r, i}$

$Y_{i}=\ln \frac{\left(\bar{E}_{r, i}\right)^{-\alpha}}{\left(F_{C d, i} \cdot R_{C d, i}-1\right) Q_{0, i}(\alpha) \cdot \frac{G_{e, i}}{G_{t h, i}}}$, para a EQ. (2.46);

ou

$Y_{i}=\ln \frac{\left(\bar{E}_{r, i}\right)^{-\alpha}\left(A_{s p, i}\right)_{C d}}{k_{0, A u}(i) \varepsilon_{p, i} F_{C d, i} Q_{0, i}(\alpha) G_{e, i}}$, para EQ. (2.47)

$\mathrm{O}$ valor de $k_{0}$ é obtida pela a EQ. (2.44). Os índices $i$ e $A u$ referem-se à amostra e ao comparador, $\mathrm{Au}$, respectivamente. $\mathrm{E} Q_{0}(\alpha)$ está relacionada conforme definida 
pela EQ. (2.63). O procedimento deste método consistiu em ajustar simultaneamente os valores de $k_{0}$, dados pela EQ. (3.5), e o valor de $\alpha$, dado pela EQ. (3.4), utilizando os valores de $Y_{i}$ expressos pela EQ. (2.47). O ajuste foi realizado pelo método dos mínimos quadrados, usando uma matriz de planejamento que vincula os valores de $k_{0}$ obtidos das medições aos valores de $k_{0}$ necessários para o cálculo do parâmetro $\alpha$.

\subsubsection{As equações de $\alpha$ e $k_{0}$}

Partindo da EQ. (3.1) e das definições de $X_{i}$ e $Y_{i}$, chegou-se a expressão:

$$
\ln \frac{\left(\bar{E}_{r, i}\right)^{-\alpha}\left(A_{s p}\right)_{C d, i}}{k_{0, A u}(i) \varepsilon_{p, i} F_{C d, i} Q_{0, i}(\alpha) G_{e, i}}=a+\alpha \ln \bar{E}_{r, i}
$$

E com uma manipulação algébrica, obteve-se:

$$
\ln \frac{\left(A_{s p, i}\right)_{C d}}{k_{0, A u}(i) \varepsilon_{p, i} F_{c d, i} G_{e p, i}}-\alpha \ln \bar{E}_{r, i}-\ln Q_{0, i}(\alpha)=a+\alpha \ln \bar{E}_{r, i}
$$

Portanto:

$$
\ln \frac{\left(A_{s p, i}\right)_{C d}}{k_{0, A u}(i) \varepsilon_{p, i} F_{c d, i} G_{e p, i}}=a+2 \alpha \ln \bar{E}_{r, i}+\ln Q_{0, i}(\alpha)
$$

O parâmetro $\alpha$ encontra-se apenas no lado direito da EQ. (3.4), entretanto, ainda não é linear em razão à expressão de $\ln Q_{0, i}(\alpha)$. Sabendo que $\alpha \approx 0$, expandiu-se a expressão de $\ln Q_{0, i}(\alpha)$ nas proximidades de $\alpha=0$, cujo detalhes da operação pode ser vista na referência ${ }^{[19]}$. Com o resultado deste procedimento obteve-se a expressão linear em $\alpha$ :

$$
\ln \frac{\left(A_{s p, i}\right)_{C d}}{k_{0, A u}(i) Q_{0, i} \varepsilon_{p, i} F_{c d, i} G_{e p, i}}=a+\frac{\alpha}{Q_{0, i}}\left[\left(Q_{0, i}+0,429\right) \ln \bar{E}_{r, i}-0,602\right]
$$


Ajustou-se a EQ. (2.44) com a EQ. (3.5), possibilitando o uso de todos os dados referentes às amostras irradiadas com e sem cádmio (incluindo para o $\mathrm{Au})$, não sendo mais necessário o uso de valores tabelados de $k_{0}$ para a EQ. (3.5):

$$
\ln \left(\frac{A_{s p, i}-\frac{\left(A_{s p, i}\right)_{C d}}{F_{C d, i}}}{A_{s p . A u}-\frac{\left(A_{s p, A u} C d\right.}{F_{C d, A u}}} \cdot \frac{G_{t h, A u .} \varepsilon_{p, A u}}{G_{t h, i .} \varepsilon_{p, i}}\right)=\ln k_{0, A u}(i)
$$

\subsubsection{Determinação de $\alpha$ e $k_{0}$ em um único ajuste}

Para ajustar os valores de $k_{0}$ usando ambas as EQ. (3.5) e (3.6), foram colocados os valores de $k_{0}$ do lado direito da EQ. (3.5) como um parâmetro ajustável:

$$
\ln \frac{\left(A_{s p, i}\right)_{C d}}{Q_{0, i} \varepsilon_{p, i} F_{c d, i} G_{e p, i}}=a+\frac{\alpha}{Q_{0, i}}\left[\left(Q_{0, i}+0,429\right) \ln \bar{E}_{r, i}-0,602\right]+k_{0, A u}(i)
$$

A EQ. (3.7), portanto, possui os parâmetros lineares $a, \alpha$ e $\ln k_{0, A u}(i) \mathrm{e}$ a EQ. (3.6) tem apenas o parâmetro linear $k_{0, A u}(i)$. Com as EQ. (3.6) e (3.7) criou-se a equação matricial do problema pela superposição das duas equações, gerando um vetor $\vec{Y}$, onde:

$$
\vec{Y}=\left[\begin{array}{l}
Y^{s u p} \\
Y^{\text {inf }}
\end{array}\right]
$$

Essa divisão da EQ. (3.8) em $Y^{\text {Sup }}$ e $Y^{\text {Inf }}$ são, respectivamente, a composição das EQ. (3.7) e (3.6) formando o vetor $\vec{Y}$, ou seja: 


$$
\vec{Y}=\left[\begin{array}{c}
\ln \frac{\left(A_{s p, i}\right)_{C d}}{Q_{0, i} \varepsilon_{p, i} F_{c d, i} G_{e p, i}} \\
\ln \left(\frac{A_{s p, i}-\frac{\left(A_{s p, i}\right) C d}{F_{C d, i}}}{A_{s p . A u}-\frac{\left(A_{s p, A u}\right)_{C d}}{F_{C d, A u}}} \cdot \frac{G_{t h, A u .} \varepsilon_{p, A u}}{G_{t h, i .} \varepsilon_{p, i}}\right)
\end{array}\right]
$$

e a equação matricial é dada por:

$$
\vec{Y}=\left[\begin{array}{c}
a+\frac{\alpha}{Q_{0, i}}\left[\left(Q_{0, i}+0,429\right) \ln \bar{E}_{r, i}-0,602\right]+k_{0, A u}(i) \\
\ln k_{0, A u}(i)
\end{array}\right]
$$

que pode ser escrita como $\vec{Y}=X \cdot \vec{A}$.

A quantidade de linhas que terá a matriz depende da quantidade de isótopos analisados. O vetor $\vec{A}$ representa os parâmetros a serem encontrados.

$$
\vec{A}=\left[\begin{array}{c}
a \\
\alpha \\
\ln k_{01} \\
\ln k_{02} \\
\vdots \\
\ln k_{0 n}
\end{array}\right]
$$

A matriz $X$ é a matriz de planejamento, montada com as derivadas em primeira ordem de cada parâmetro de $\vec{A}$ : 


$$
X=\left[\begin{array}{cccccc}
1 & \frac{1}{Q_{0,1}}\left[\left(Q_{0,1}+0,429\right) \ln \vec{E} r_{, 1}-0,602\right] & 1 & 0 & \cdots & 0 \\
1 & \frac{1}{Q_{0,2}}\left[\left(Q_{0,2}+0,429\right) \ln \vec{E}_{r, 2}-0,602\right] & 0 & 1 & \cdots & 0 \\
& \vdots & \vdots & \vdots & \vdots & \vdots \\
1 & \frac{1}{Q_{0, n}}\left[\left(Q_{0, n}+0,429\right) \ln \vec{E}_{r, n}-0,602\right] & 0 & 0 & \cdots & 1 \\
0 & 0 & 1 & 0 & \cdots & 0 \\
0 & 0 & 0 & 1 & \cdots & 0 \\
\vdots & \vdots & \vdots & \vdots & \vdots & \vdots \\
0 & 0 & 0 & 0 & \cdots & 1
\end{array}\right]
$$

Criou-se ainda a matriz $V_{y}$ que contém os termos da diagonal principal, correspondentes às variâncias dos elementos de $\vec{Y}$, e também os termos cruzados, que correspondem às covariâncias entre os diferentes elementos de $\vec{Y}$. Essa matriz mostrou-se trabalhosa pelo fato de haver vários fatores em comum nos diferentes elementos de $\vec{Y}$, tornando uma matriz grande e complexa.

Os elementos da diagonal principal de $V_{y}$ foram obtidos pela propagação das variâncias dos elementos de $\vec{Y}$. As covariâncias foram calculadas conforme os critérios de avaliação: o envolvimento dos parâmetros do mesmo isótopo com mesma transição gama; o mesmo isótopo, mas com transições gama diferentes e isótopos diferentes.

Uma vez montadas as matrizes $\vec{Y}, V_{y}$ e $X$, foi possível calcular os parâmetros de $\vec{A}$ e as respectivas covariâncias $V_{A}$ por meio do método dos mínimos quadrados, utilizando o formalismo matricial, expresso da forma:

$$
V_{A}=\left(X^{t} V_{y} X\right)^{-1}
$$

e

$$
\vec{A}=V_{A} X^{t} V_{y}^{-1} \vec{Y}
$$


Os valores de $\vec{Y}$ ajustados são, portanto:

$$
\vec{Y}_{a j u}=X \vec{A}
$$

Realizou-se a diferença entre os valores ajustados e experimentais para a realização do $\chi^{2}$, dado:

$$
\vec{D}=\vec{Y}-\vec{Y}_{a j u}
$$

Assim:

$$
\chi^{2}=\vec{D}^{t} V_{y}^{-1} \vec{D}
$$

Desse modo, concluiu-se o ajuste proposto, podendo analisar e comparar os resultados de $k_{0}$ obtidos com os da literatura.

\subsubsection{Transferência do erro de $X$ para $Y$}

As grandezas $\bar{E}_{r, i}$ e $Q_{0, i}$ possuem incertezas e estão presentes também em $X$. Na propagação das incertezas de $X$ para $Y$, após a determinação do valor de $\alpha$, uma nova matriz $V_{Y}$ é calculada. Os termos da diagonal principal da matriz correspondem a novos valores de variâncias dos $Y_{i}, \sigma_{Y_{i}}^{\prime}$, calculados por:

$$
\sigma_{Y_{i}}^{\prime 2}=\sigma_{Y_{i}}^{2}+\left(\frac{\delta Y_{i}}{\delta \bar{E}_{r, i}}\right)^{2} \sigma_{\bar{E}_{r, i}}^{2}+\left(\frac{\delta Y_{i}}{\delta Q_{0, i}}\right)^{2} \sigma_{Q_{0, i}}^{2}
$$

cujo resultado é:

$$
\sigma_{Y_{i}}^{\prime 2}=\sigma_{Y_{i}}^{2}+\left[\frac{\alpha\left(Q_{0, i}+0,429\right)}{\bar{E}_{r, i} Q_{0, i}}\right]^{2} \sigma_{\bar{E}_{r, i}}^{2}+\left\{\frac{\alpha \ln \bar{E}_{r, i}}{Q_{0, i}}-\frac{\alpha\left[\ln \bar{E}_{r, i}\left(Q_{0, i}+0,429\right)-0,602\right]}{Q_{0, i}^{2}}\right\}^{2} \sigma_{Q_{0, i}}^{2}
$$

Como $\bar{E}_{r, i}$ e $Q_{0, i}$ têm os mesmos valores para energias gamas diferentes do mesmo isótopo, é necessário também transferir estas incertezas para os termos covariantes de $V_{y}$. Os elementos de $V_{Y}$ correspondentes a $Y^{\text {Sup }}$ do mesmo isótopo devem ser recalculados. 


\subsubsection{Ajuste não linear de $\alpha$ e $k_{0}$}

Criou-se um novo algoritmo para o ajuste de $\alpha$ e $k_{0}$, passando agora para um ajuste não linear, esperando-se que seja obtido o mesmo resultado do método linear. Um procedimento utilizado em funções não lineares é o método de Gauss-Marquardt ${ }^{20]}$. No método, expande-se a função a ser ajustada até a primeira ordem da série de Taylor, e escolhe-se arbitrariamente os valores iniciais para os parâmetros. Ajustam-se então as variações nos parâmetros a partir dos valores escolhidos, encontrando-se as variações que produzam o mínimo da soma dos quadrados dos resíduos ${ }^{[20]}$.

Assim, com o método de Gauss-Marquardt criou-se uma matriz $R(\lambda)$ onde:

$$
R(\lambda)_{i j}=\left(1+\lambda \delta_{i j}\right)\left(X^{t} V_{y}^{-1} X\right)_{i j}
$$

sendo $\delta_{i j}=1$ se $i=j ; \delta_{i j}=0$ se $i \neq j$

Isso significa que a matriz $R$ é igual à matriz $\left(X^{t} V_{y}^{-1} X\right)$ com a diagonal multiplicada por $1+\lambda$. Assim, o procedimento consiste, resumidamente, em criar uma matriz $\vec{A}^{\prime}$ para os parâmetros a serem ajustados e seu $\chi_{\text {inical }}^{2}$. Calcular posteriormente os valores $\vec{A}^{\text {novo }}$ dado por:

$$
\vec{A}^{\text {novo }}=\vec{A}^{\prime}+\Delta \vec{A}
$$

onde:

$$
\Delta \vec{A}=R(\lambda)_{i j} X^{t} V_{y}^{-1} Y^{t}
$$

Calcular um $\chi_{\text {novo }}^{2}$ e realizar a diferença entre eles, até que se verifique que $\chi_{\text {novo }}^{2}<\chi_{\text {inicial }}^{2}$. Em caso contrário, multiplica-se $\lambda$ por um fator (usualmente 10) e recalcula-se a matriz $R(\lambda)$. Quando verificado que $\chi_{\text {novo }}^{2}<\chi_{\text {inicial }}^{2}$, reduz-se 0 valor de $\lambda$ pelo fator multiplicativo, calcula-se um novo $R(\lambda)$ e atualiza-se os parâmetros pela EQ. (3.24), substitui-se o $\chi^{2}$ atual pelo novo $\chi^{2}$ calculado e repete-se o procedimento até que as diferenças entre os $\chi^{2}$ possam ser 
ignorados. Em outras palavras, significa descartar os valores do novo ajuste quando o chi-quadrado ajustado é maior que o anterior (ou inicial) ou substituir o valor anterior pelo novo quando o último chi-quadrado ajustado é menor, até que se chegue em uma diferença considerada desprezível entre eles. Estes foram os procedimentos executados para a elaboração do código de ajuste único, AKFIT v2.1, no presente trabalho.

\subsection{Programas utilizados}

\subsubsection{Código COVAR}

Foi utilizado a linguagem de programação Fortran ${ }^{[79,80]}$ para a criação do código. É caraterizado por sua linguagem matemática, cujo nome é um acrônimo da expressão "IBM Mathematical Formula Translation System'. Costuma-se fazer uma pequena distinção entre FORTRAN e Fortran, no qual a primeira, em caixa alta, representa as versões iniciais da linguagem, enquanto a segunda representa as versões mais recentes, a partir do Fortran 90. A linguagem Fortran é principalmente usada em Ciência da Computação e Análise Numérica. Apesar de ter sido inicialmente uma linguagem de programação procedural, versões recentes de Fortran possuem características que permitem suportar programação orientada por objetos.

Existem diversos programas compiladores de Fortran. Procurou-se, neste presente trabalho, utilizar ferramentas que fossem grátis ou de código aberto (open source). Para compiladores utilizou-se o compilador GNU Fortran V. $6^{[81]}$. Entretanto, por se tratar de um compilador Linux, necessitou-se a instalação de um emulador de programas deste sistema operacional para sistemas Windows 64 bits. Neste caso, foi utilizado o Cygwin V. 2.8.0-1[82].

Para o ambiente de desenvolvimento integrado (IDE) empregou-se 0 programa Eclipse[83], em sua versão Neon, com o plugin Photran, um plugin IDE voltado para Fortran que suporta as versões desde 77 até 2008 da linguagem, e que integra o compilador GNU Fortran ao Eclipse. 


\subsubsection{Código AKFIT}

O código AKFIT foi desenvolvido no ambiente do programa científico para Computação Numérica Scilab V. 6.0.0 ${ }^{[84]}$. Esta linguagem de alto nível e orientada à Análise Numérica, semelhante ao Matlab, provê um ambiente para interpretação com diversas ferramentas. Algoritmos complexos podem ser criados em poucas linhas de código, em comparação com outras linguagens como C, Fortran ou $\mathrm{C}++$.

Scilab inclui extensas funções matemáticas com a possibilidade de adicionar interativamente programas de várias linguagens (Fortran, $\mathrm{C}, \mathrm{C}++$, Java). Possui sofisticadas estruturas de dados, incluindo listas, polinômios e sistemas lineares, e um interpretador de linguagem de programação de alto nível, podendo o usuário definir novos tipos de informações e operações sobre essas estruturas.

A primeira versão do AKFIT realizava apenas um ajuste linear para a determinação de $\alpha$ e $k_{0}$. Criou-se um ajuste não-linear baseado no método de Gauss-Marquardt ${ }^{[20]}$, atualizando para sua segunda versão, AKFIT v2. A implementação do ajuste não-linear no código foi motivada em razão de analisar a capacidade do método em comparação com o método linear. Se verificado que o método não-linear produz os mesmos resultados que o linear, será possível desenvolver ajustes mais complexos, incluindo mais parâmetros que envolvem o método $k_{0}$, como o parâmetro $Q_{0}$ e $f$. Por fim, AKFIT v2 ainda possuía algumas limitações. O ajuste criado apenas trabalhava com um único comparador $A u$, ou seja, só era possível realizar ajustes para uma única irradiação. O código se tornava inútil quando havia análises com elementos de diferentes irradiações, com diferentes comparadores de Au. Por este motivo, foi implementado um recurso que possibilitou a utilização de vários comparadores que, consequentemente, tornou-se possível realizar análises com várias irradiações distintas. Em razão desta implementação, AKFIT foi atualizado para a versão 2.1.

\subsubsection{Análises dos espectros e dados experimentais}

A aquisição dos espectros foi realizada por meio de uma placa de aquisição de dados de marca Ortec Modelo ACE, de 8192 canais. Foi também utilizado o programa MAESTRO, um analisador multicanal (MCA) compatível com sistemas de espectroscopia gama e alfa e com uma ampla variedade de 
aplicações científicas no meio industrial, ensino e afins. MAESTRO é uma excelente escolha para aplicações de espectroscopia básica e acompanha, por padrão, na maioria dos analisadores multicanais da Ortec. Com MAESTRO podese observar uma irradiação em tempo real, verificar os picos e as propriedades do hardware. O registro do espectro da irradiação realizada é salvo no formato próprio da Ortec, $\mathrm{CHN}$.

Os espectros analisados no presente trabalho são os mesmos obtidos anteriormente ${ }^{[18]}$ para possibilitar uma comparação direta entre os resultados de $k_{0}, \alpha$ e $Q_{0}$ obtidos com método desenvolvido no presente trabalho com aqueles obtidos com o método convencional.

O cálculo das eficiências de detecção para cada linha de emissão gama das fontes padrões medidas foi efetuado por meio do programa ALPINO[85]. Este programa, elaborado em linguagem FORTRAN, calcula a atividade ou eficiência de detecção (segundo opções de entrada) para cada energia gama da fonte medida, diretamente a partir do espectro obtido. Além disso, este programa permite calcular (ou incluir nos cálculos) a atividade e a intensidade do pico de absorção total de energia $N_{p}$ (com ou sem a subtração da radiação de fundo), determinar os limites dos canais do pico de interesse e correção do tempo morto.

O programa ALPINO utiliza um método de integração numérica para a área sob o pico de absorção total. $O$ centroide é identificado por meio da calibração de canais em função da energia. As posições dos canais $\left(K_{i}\right)$ que definem as regiões de contagem de fundo e de pico são dadas por:

- $\quad K_{p}$ - Canal do centroide;

- $\quad K_{1}$ - Início da contagem de fundo;

- $K_{2}$ - Início do pico de absorção total;

- $\quad K_{3}-$ Fim do pico de absorção total;

- $K_{4}$ - Fim da contagem de fundo.

A FIG. 4 apresenta um exemplo do método de integração numérica utilizado: 


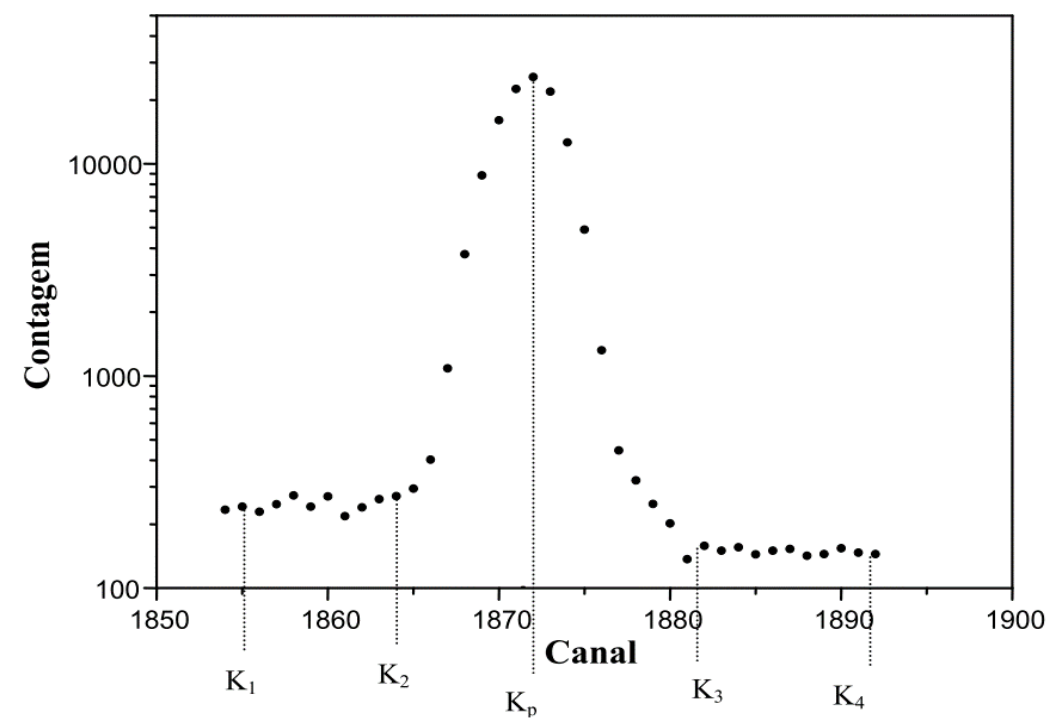

FIGURA 4 - Exemplo dos parâmetros utilizados no método de integração numérica da área sob o pico de absorção total[ ${ }^{[18]}$.

Adquiridos os dados da análise dos espectros desejado pelo MAESTRO, e com as informações de cada fonte padronizada de interesse e suas transições gama, criou-se um arquivo de dados de entrada para o ALPINO. O arquivo contém informações como tempo de medida, datas e horas iniciais (de referência) e de medida da fonte radioativa, canais de cada pico e largura meia altura, FWHM (caso necessário), meia vida, abundância absoluta do raio-gama considerado, massa, entre outros dados de processamento.

Utilizou-se o programa criado no CRPq, LOGFIT, para o cálculo da curva de eficiência após a execução do ALPINO para todas as fontes padronizadas de interesse. O LOGFIT executa um ajuste em escala log-log com as eficiências das fontes padrões obtidas pelo ALPINO e gera um arquivo de saída com os valores das eficiências para as energias gamas de interesse, que seriam, neste caso, as amostras. Este programa de ajuste aplica a metodologia de Matriz de Covariâncias. O ALPINO é mais uma vez executado, dessa vez para as amostras e não para as fontes padrões, com suas eficiências obtidas pelo LOGFIT incluídas no arquivo de dados.

É importante destacar que os valores das auto-blindagens para nêutrons térmicos e epitérmicos $G_{t h}$ e $G_{e}$, fator de cádmio $F_{C d}$ e a razão do fluxo de nêutrons térmicos e epitérmicos $f$, foram calculados por outros meios, conforme descrito na referência ${ }^{[18]}$. Com todos os resultados adquiridos para cada 
amostra e seu comparador foi possível criar os arquivos de dados de entrada para os códigos COVAR v4.1 e AKFIT v2.1. Ambos os códigos, apesar de utilizarem um arquivo de dados de entrada semelhante, não são compatíveis de execução um com o outro. Enquanto COVAR v4.1 calcula a taxa de contagem específica dos elementos com e sem cádmio $A_{s p, i}$ e $\left(A_{s p}\right)_{C d, i}$, e necessita do valor de $\alpha$ como dado de entrada, o AKFIT v2.1 utiliza as taxas de contagens específicas previamente calculadas e determina seu próprio valor de $\alpha$.

\subsection{Caracterização das amostras}

Duas irradiações em diferentes períodos foram utilizadas no presente trabalho[18]. A primeira irradiação foi realizada no ano de 2008 , com os alvos ${ }^{197} \mathrm{Au}$ (liga de Al 0,10\%), ${ }^{94} \mathrm{Zr},{ }^{64} \mathrm{Zn},{ }^{45} \mathrm{Sc}$ e ${ }^{139} \mathrm{La}$ (liga de Al 0,665\%). A segunda irradiação foi realizada no ano de 2010 , com os alvos ${ }^{197} \mathrm{Au}$ (liga de Al 0,13\%), ${ }^{94} \mathrm{Zr},{ }^{96} \mathrm{Zr},{ }^{64} \mathrm{Zn},{ }^{68} \mathrm{Zn},{ }^{45} \mathrm{Sc}$ e ${ }^{139} \mathrm{La},{ }^{59} \mathrm{Co}$ (liga de Al 0,475\%). As reações geradas para cada caso foram: ${ }^{94} \mathrm{Zr}(\mathrm{n}, \gamma){ }^{95} \mathrm{Zr},{ }^{64} \mathrm{Zn}(\mathrm{n}, \gamma){ }^{65} \mathrm{Zn},{ }^{68} \mathrm{Zn}(\mathrm{n}, \gamma){ }^{69 m} \mathrm{Zn},{ }^{139} \mathrm{La}(\mathrm{n}, \gamma){ }^{140} \mathrm{La}$, ${ }^{45} \mathrm{Sc}(\mathrm{n}, \gamma)^{46} \mathrm{Sc}$ e ${ }^{59} \mathrm{Co}(\mathrm{n}, \gamma)^{60} \mathrm{Co}$. Os dados utilizados dos produtos das reações do ano de 2008 estão apresentados na TAB. 1; os valores em parênteses representam as incertezas nas casas decimais:

TABELA 1 - Dados característicos sobre probabilidade de emissão gama, meiavida, espessura e concentração da liga de cada produto resultante das irradiações no ano de $2008^{[86,11]}$.

\begin{tabular}{|c|c|c|c|c|}
\hline \multicolumn{5}{|c|}{ Características das reações de 2008} \\
\hline Produto- $E_{\gamma}(\mathrm{keV})$ & $\mathbf{I}(\%)$ & $T_{1 / 2}(D)$ & $x($ fio $-\mathrm{mm})$ & Liga (\%) \\
\hline \multicolumn{5}{|l|}{${ }^{198} \mathrm{Au}$} \\
\hline 411,8 & $95,62(6)$ & 2,69 (3) & 1 & 0,1 \\
\hline \multicolumn{5}{|l|}{${ }^{95} \mathrm{Zr}$} \\
\hline $\begin{array}{l}724,2 \\
756,7\end{array}$ & $\begin{array}{l}44,27(13) \\
54,38(22)\end{array}$ & $64,03(6)$ & 0,096 & 100 \\
\hline \multicolumn{5}{|l|}{${ }^{65} \mathrm{Zn}$} \\
\hline 1115,5 & $50,22(11)$ & $244,01(9)$ & 0,9 & 100 \\
\hline \multicolumn{5}{|l|}{${ }^{46} \mathrm{Sc}$} \\
\hline $\begin{array}{r}889,3 \\
1120,5\end{array}$ & $\begin{array}{c}99,9837(3) \\
99,97(2)\end{array}$ & $83,79(16)$ & 0,13 & 100 \\
\hline \multicolumn{5}{|l|}{${ }^{140} \mathrm{La}$} \\
\hline 328,7 & $20,8(3)$ & & & \\
\hline 487,0 & $46,1(5)$ & $1.68(21)$ & 1 & 0.665 \\
\hline 815,8 & $23,72(20)$ & $1,68(21)$ & 1 & \\
\hline 1596,2 & $95,40(5)$ & & & \\
\hline
\end{tabular}


Para os produtos das reações das irradiações no ano de 2010, os dados estão apresentados na TAB. 2; os valores em parênteses representam as incertezas nas casas decimais:

TABELA 2 - Dados característicos sobre probabilidade de emissão gama, meiavida, espessura e concentração da liga das reações dos dados de 2010[86, 11].

\begin{tabular}{|c|c|c|c|c|}
\hline \multicolumn{5}{|c|}{ Características das reações de 2010} \\
\hline Produto- $E_{\gamma}(\mathrm{keV})$ & $\mathbf{I}(\%)$ & $T_{1 / 2}(D)$ & $x($ fio - mm) & Liga (\%) \\
\hline \multicolumn{5}{|l|}{${ }^{198} \mathrm{Au}$} \\
\hline 411,8 & $95,62(6)$ & $2,69(3)$ & 0,51 & 0,13 \\
\hline \multicolumn{5}{|l|}{${ }^{95} \mathrm{Zr}$} \\
\hline $\begin{array}{l}724,2 \\
756,7\end{array}$ & $\begin{array}{l}44,27(13) \\
54,38(22)\end{array}$ & $64,03(6)$ & 0,25 & 100 \\
\hline \multicolumn{5}{|l|}{${ }^{65} \mathrm{Zn}$} \\
\hline 1115,5 & $50,22(11)$ & $244,01(9)$ & 0,85 & 100 \\
\hline \multicolumn{5}{|l|}{ 69m Zn } \\
\hline 438,6 & $94,77(20)$ & $0,57(2)$ & 0,85 & 100 \\
\hline \multicolumn{5}{|l|}{${ }^{46} \mathrm{Sc}$} \\
\hline $\begin{array}{r}889,3 \\
1120,5\end{array}$ & $\begin{array}{c}99,9837(3) \\
99,97(2)\end{array}$ & $83,79(16)$ & 0,13 & 100 \\
\hline \multicolumn{5}{|l|}{${ }^{140} \mathrm{La}$} \\
\hline 328,7 & $20,8(3)$ & & & \\
\hline 487,0 & $46,1(5)$ & & & \\
\hline 815,8 & $23,72(20)$ & $1,68(21)$ & 0,64 & 0,665 \\
\hline 1596,2 & $95,40(5)$ & & & \\
\hline \multicolumn{5}{|l|}{${ }^{60} \mathrm{Co}$} \\
\hline $\begin{array}{l}1173,2 \\
1332,5\end{array}$ & $\begin{array}{c}99,85(3) \\
99,9826(6)\end{array}$ & $1923,95(22)$ & 0,76 & 0,475 \\
\hline
\end{tabular}

A TAB. 3 apresenta as massas (w) das reações com e sem cádmio irradiados nos anos de 2008 e 2010; os valores em parênteses representam as incertezas nas casas decimais:

TABELA 3 - Valores das massas e suas incertezas para cada reação nos anos de 2008 e 2010.

\begin{tabular}{cccccc}
\hline $\begin{array}{c}\text { Produto } \\
(\mathbf{2 0 0 8})\end{array}$ & $(w)_{\mathrm{Cd}}(\mathbf{g})^{[18]}$ & $w(\mathbf{g})^{[18]}$ & $\begin{array}{c}\text { Produto } \\
(\mathbf{2 0 1 0})\end{array}$ & $(w)_{\mathrm{Cd}}(\mathbf{g})^{[18]}$ & $w(\mathbf{g})^{[18]}$ \\
\hline${ }^{198} \mathrm{Au}$ & $0,00960(20)$ & $0,00800(20)$ & ${ }^{198} \mathrm{Au}$ & $0,00610(10)$ & $0,00600(10)$ \\
\hline${ }^{95} \mathrm{Zr}$ & $0,00560(10)$ & $0,00510(10)$ & ${ }^{95} \mathbf{Z r}$ & $0,00470(9)$ & $0,00410(8)$ \\
${ }^{65} \mathrm{Zn}$ & $0,2492(4)$ & $0,2360(4)$ & ${ }^{65} \mathbf{Z n}$ & $0,2492(5)$ & $0,2368(5)$ \\
\hline${ }^{46} \mathrm{Sc}$ & $0,01240(17)$ & $0,00140(14)$ & ${ }^{69 m} \mathrm{Zn}$ & $0,2492(5)$ & $0,2368(5)$ \\
\hline${ }^{140} \mathrm{La}$ & $0,00930(3)$ & $0,00840(3)$ & ${ }^{46} \mathrm{Sc}$ & $0,01290(20)$ & $0,00090(3)$ \\
\hline & & & ${ }^{140} \mathrm{La}$ & $0,00540(10)$ & $0,00530(10)$ \\
\hline & & & ${ }^{60} \mathrm{Co}$ & $0,0159(30)$ & $0,0150(30)$ \\
\hline
\end{tabular}




\subsubsection{Caracterização das fontes padronizadas e curva de eficiência}

As fontes padronizadas utilizadas para a geração da curva de eficiência do detector foram ${ }^{59} \mathrm{Co},{ }^{132} \mathrm{Ba},{ }^{136} \mathrm{Cs} \mathrm{e}{ }^{151} \mathrm{Eu}$, fornecidas pela Agência Internacional de Energia Atômica (AIEA). As características do produto das reações das fontes, como meia-vida, transição gama considerada e sua probabilidade, atividade e a correção do efeito de soma em cascata estão representadas na TAB. 4:

TABELA 4 - Características das reações das fontes padronizadas utilizadas para o cálculo da curva de eficiência ${ }^{[86,87]}$.

\begin{tabular}{|c|c|c|c|}
\hline \multicolumn{4}{|c|}{ Características das reações das fontes padrões } \\
\hline Produto- $E_{\gamma}(\mathrm{keV})$ & $I \gamma(\%)$ & $T_{1 / 2}(\mathrm{D})$ & Atividade (kBq) \\
\hline \multicolumn{4}{|l|}{${ }^{133} \mathrm{Ba}$} \\
\hline 276,4 & $7,13(6)$ & \multirow{3}{*}{$3862(15)$} & \multirow{3}{*}{$376,0(45)$} \\
\hline 302,85 & $18,31(11)$ & & \\
\hline 356,02 & $62,05(6)$ & & \\
\hline \multicolumn{4}{|l|}{${ }^{152} \mathrm{Eu}$} \\
\hline 244,70 & $7,55(4)$ & \multirow{8}{*}{ 4933(11) } & \multirow{8}{*}{$404,0(65)$} \\
\hline 344,28 & $26,59(12)$ & & \\
\hline 443,96 & $2,80(2)$ & & \\
\hline 778,90 & $12,97(6)$ & & \\
\hline 867,39 & $4,243(23)$ & & \\
\hline 964,05 & $14,50(6)$ & & \\
\hline 1112,10 & $13,41(6)$ & & \\
\hline 1408,01 & $20,85(8)$ & & \\
\hline \multicolumn{4}{|l|}{${ }^{60} \mathrm{Co}$} \\
\hline 1173,20 & $99,85(3)$ & \multirow{2}{*}{$1923,95(22)$} & \multirow{2}{*}{$379,2(19)$} \\
\hline 1332,50 & $99,9826(6)$ & & \\
\hline \multicolumn{4}{|l|}{${ }^{137} \mathrm{Cs}$} \\
\hline 661,66 & $84,99(20)$ & $1102(6)$ & $406,5(4)$ \\
\hline
\end{tabular}

Em razão deste presente trabalho apresentar irradiações já efetuadas e analisadas em seus respectivos anos (2008 e 2010), optou-se aqui em apenas reproduzir os mesmos procedimentos realizados anteriormente, a fim de, em princípio, compreender todos os processos essenciais, facilitando o desenvolvimento dos códigos aqui propostos.

Executou-se o programa ALPINO, configurado para calcular as eficiências de cada fonte padrão. Incluiu-se, ao término das execuções, os valores das eficiências no programa LOGFIT, com objetivo de determinar a curva de eficiência do detector. Com o arquivo de saída obtido pelo LOGFIT adicionou-se 
as eficiências de cada transição gama das amostras obtida pela curva. $O$ programa ALPINO foi configurado não mais para calcular as eficiências, mas para ler os valores das eficiências incluídas nos arquivos de entrada de cada amostra, além de calcular suas atividades e as áreas dos picos de absorção total de cada transição gama considerada.

Os valores de $F_{C d}, G_{T h}$ e $G_{e}$ foram adquiridos por meio de resultados já calculados pelo LMN antes do desenvolvimento do presente trabalho. Para os valores das energias de ressonância efetiva $\bar{E}_{r, i}$ de cada amostra, foi obtida pelo banco de dados eletrônico Compilation of $k_{0}$ and Related Data for $N A A$, da International Union of Pure and Applied Chemistry (IUPAC), em sua versão 4, apresentado em 2004[88]. Para COVAR 4.1 ainda necessitou-se obter previamente os valores de $Q_{0}, f$ e $\alpha$. Os valores de $Q_{0}$ também foram adquiridos pelo banco de dados eletrônico da IUPAC, enquanto $f$ e $\alpha$ foram obtidos pelas referências de Dias et $a^{[9,17]}$. Já para AKFIT v2.1, necessitou-se conhecer os valores das taxas de contagens específicas com e sem cádmio, $A_{S p, i}$ e $\left(A_{S p}\right)_{C d, i}$. Estes valores eram possíveis de serem encontrados por meio de um cálculo externo e, posteriormente, incluídos no arquivo de entrada ou por meio dos resultados obtidos pelo arquivo de saída do COVAR v4.1. Para que seja feita uma análise independente dos códigos, decidiu-se calcular $A_{S p, i}$ e $\left(A_{S p}\right)_{C d, i}$ externamente, ou seja, de modo manual. Os parâmetros explicitados e seus valores estão apresentados na TAB. 5. Os valores em parênteses representam as incertezas nas casas decimais. 
TABELA 5 - Valores de fator de cádmio, correção de auto-blindagem para nêutrons térmicos e epitérmicos, energia de ressonância efetiva, razão entre fluxo térmico e epitérmico, alfa e $Q_{0}$, para as reações de 2008 e 2010[18].

\begin{tabular}{lccccccc}
\hline \multicolumn{7}{c}{ Parâmetros das reações (2008 e 2010) } \\
\hline Produto & $\boldsymbol{F}_{\boldsymbol{C d}}$ & $\boldsymbol{G}_{\boldsymbol{t h}}$ & $\boldsymbol{G}_{\boldsymbol{e}}$ & $\overline{\boldsymbol{E}}_{\boldsymbol{r}, \boldsymbol{i}}$ & $\boldsymbol{f}$ & $\boldsymbol{\alpha}$ & $\boldsymbol{Q}_{0}$ \\
\hline${ }^{198} \mathrm{Au}$ & $0,9989(4)$ & 1,0000 & $0,9808(4)$ & $5,65(2)$ & $49,7(19)$ & $-0,001(31)$ & $15,71(29)$ \\
\hline${ }^{95} \mathbf{Z r}$ & $0,9968(7)$ & 1,0000 & $0,9990(4)$ & $6,26(25) \times 10^{3}$ & $49,7(19)$ & $-0,001(31)$ & $5,31(17)$ \\
\hline${ }^{65} \mathbf{Z n}$ & $0,9928(19)$ & 0,9924 & $0,9605(87)$ & $2,56(26) \times 10^{3}$ & $49,7(19)$ & $-0,001(31)$ & $1,91(9)$ \\
\hline${ }^{69} \mathrm{mZn}$ & $1,0000(50)$ & 0,9993 & $0,9564(91)$ & $590(59)$ & $49,7(19)$ & $-0,001(31)$ & $2.56(8)$ \\
${ }^{140} \mathrm{La}$ & $1,0160(24)$ & 1,0000 & $0,9981(4)$ & $76(3)$ & $49,7(19)$ & $-0,001(31)$ & $1,24(7)$ \\
\hline${ }^{60} \mathrm{Co}$ & $0,9909(20)$ & 1,0000 & $0,9971(6)$ & $136(7)$ & $49,7(19)$ & $-0,001(31)$ & $1,99(6)$ \\
${ }^{46} \mathrm{Sc}$ & $1,0260(50)$ & 1,0000 & $0,9858(29)$ & $5,13(26) \times 10^{3}$ & $49,7(19)$ & $-0,001(31)$ & $0,430(26)$ \\
\hline
\end{tabular}

As TAB. 6 e 7 apresentam os valores calculados no presente trabalho para as taxas de contagens específicas para amostras com e sem cádmio de cada transição gama nas irradiações realizadas nos anos de 2008 e 2010, respectivamente.

TABELA 6 - Valores das taxas de contagens específicas com e sem Cd para as reações de 2008.

Taxas de contagens específicas para as reações de 2008

\begin{tabular}{|c|c|c|}
\hline Produto- $E_{\gamma}(\mathrm{keV})$ & $A_{S p}\left(s^{-1} g^{-1}\right)$ & $\left(A_{S p}\right)_{C d}\left(s^{-1} g^{-1}\right)$ \\
\hline${ }^{198} \mathrm{Au}$ & & \\
\hline 411,8 & $6,78(5) \times 10^{9}$ & $1,690(20) \times 10^{9}$ \\
\hline${ }^{95} \mathrm{Zr}$ & & \\
\hline 724,2 & $2,960(20) \times 10^{5}$ & $3,51(3) \times 10^{4}$ \\
\hline 756,7 & $3,550(20) \times 10^{5}$ & $3,94(3) \times 10^{4}$ \\
\hline${ }^{65} \mathrm{Zn}$ & & \\
\hline 1115,5 & $1,121(10) \times 10^{7}$ & $4,98(3) \times 10^{5}$ \\
\hline${ }^{46} \mathrm{Sc}$ & & \\
\hline 889,3 & $2,85(4) \times 10^{9}$ & $3,040(20) \times 10^{7}$ \\
\hline 1120,5 & $2,32(3) \times 10^{9}$ & $2,471(15) \times 10^{7}$ \\
\hline${ }^{140} \mathrm{La}$ & & \\
\hline 328,7 & $1,874(10) \times 10^{8}$ & $5,48(4) \times 10^{6}$ \\
\hline 487,0 & $2,790(20) \times 10^{8}$ & $8,05(6) \times 10^{6}$ \\
\hline 815,8 & $8,74(4) \times 10^{7}$ & $2,780(20) \times 10^{6}$ \\
\hline 1596,2 & $1,890(12) \times 10^{8}$ & $5,54(4) \times 10^{6}$ \\
\hline
\end{tabular}


TABELA 7 - Valores das taxas de contagens específicas com e sem cádmio e razão de cádmio para as irradiações de 2010.

Taxas de contagens específicas para as reações de 2010

\begin{tabular}{|c|c|c|}
\hline Produto- $E_{\gamma}(\mathrm{keV})$ & $A_{S p}\left(s^{-1} g^{-1}\right)$ & $\left(A_{S p}\right)_{C d}\left(s^{-1} g^{-1}\right)$ \\
\hline \multicolumn{3}{|l|}{${ }^{198} \mathrm{Au}$} \\
\hline 411,8 & $1,0121(32) \times 10^{10}$ & $2,399(6) \times 10^{9}$ \\
\hline \multicolumn{3}{|l|}{${ }^{95} \mathrm{Zr}$} \\
\hline 724,2 & $4,298(11) \times 10^{5}$ & $3,890(3) \times 10^{4}$ \\
\hline 756,7 & $5,090(12) \times 10^{5}$ & $4,667(4) \times 10^{4}$ \\
\hline \multicolumn{3}{|l|}{${ }^{65} \mathrm{Zn}$} \\
\hline 1115,5 & $1,632(4) \times 10^{7}$ & $5,581(7) \times 10^{5}$ \\
\hline \multicolumn{3}{|l|}{ 69m Zn } \\
\hline 438,6 & $2,97(4) \times 10^{6}$ & $1,29(8) \times 10^{5}$ \\
\hline \multicolumn{3}{|l|}{${ }^{46} \mathrm{Sc}$} \\
\hline 889,3 & $4,458(15) \times 10^{9}$ & $4,514(22) \times 10^{7}$ \\
\hline 1120,5 & $3,599(12) \times 10^{9}$ & $3,682(18) \times 10^{7}$ \\
\hline \multicolumn{3}{|l|}{${ }^{140} \mathrm{La}$} \\
\hline 328,7 & $2,886(6) \times 10^{8}$ & $6,92(3) \times 10^{6}$ \\
\hline 487,0 & $4,242(8) \times 10^{8}$ & $1,122(3) \times 10^{7}$ \\
\hline 815,8 & $1,324(4) \times 10^{8}$ & $3,209(20) \times 10^{6}$ \\
\hline 1596,2 & $2,866(6) \times 10^{8}$ & $6,914(24) \times 10^{6}$ \\
\hline \multicolumn{3}{|l|}{${ }^{60} \mathrm{Co}$} \\
\hline 1173,2 & $3,95(4) \times 10^{9}$ & $1,445(15) \times 10^{8}$ \\
\hline 1332,5 & $3,40(3) \times 10^{9}$ & $1,280(13) \times 10^{8}$ \\
\hline
\end{tabular}




\section{RESULTADOS E DISCUSSÃO}

\subsection{Curva de eficiência do detector HPGe}

A TAB. 8 apresenta os parâmetros obtidos no presente trabalho para 0 ajuste da curva de eficiência do Detector HPGe por meio do programa LOGFIT:

TABELA 8 - Dados do ajuste da curva de eficiência do detector HPGe realizado pelo programa LOGFIT.

\begin{tabular}{cccccc}
\hline & \multicolumn{3}{c}{ Dados do ajuste LOGFIT } \\
Parâmetro & Valor & \multicolumn{3}{c}{ Matriz de covariância } \\
\hline $\boldsymbol{a}_{\mathbf{0}}$ & $-5,984024$ & 10,7091 & & & \\
$\boldsymbol{a}_{\mathbf{1}}$ & 1,901061 & $-5,0709$ & 2,4032 & & \\
$\boldsymbol{a}_{\mathbf{2}}$ & $-0,516355$ & 0,7967 & $-0,3779$ & 0,0595 & \\
$\boldsymbol{a}_{\mathbf{3}}$ & 0,030199 & $-0,0415$ & 0,0197 & $-0,0031$ & 0,0002 \\
$\chi_{\text {red }}^{2}$ & 1,2 & & & & \\
\hline
\end{tabular}

Observa-se que o ajuste é um polinômio de grau 3 e o chi-quadrado reduzido foi de 1,2, indicando um ajuste dentro do aceitável. A curva de eficiência pode ser observada pela FIG. 5. Para ambas as irradiações, os valores das eficiências foram os mesmos, portanto, a curva da FIG. 5 é aplicável ás irradiações de 2008 e 2010 e todas as eficiências calculadas estão apresentadas na TAB. 9. 


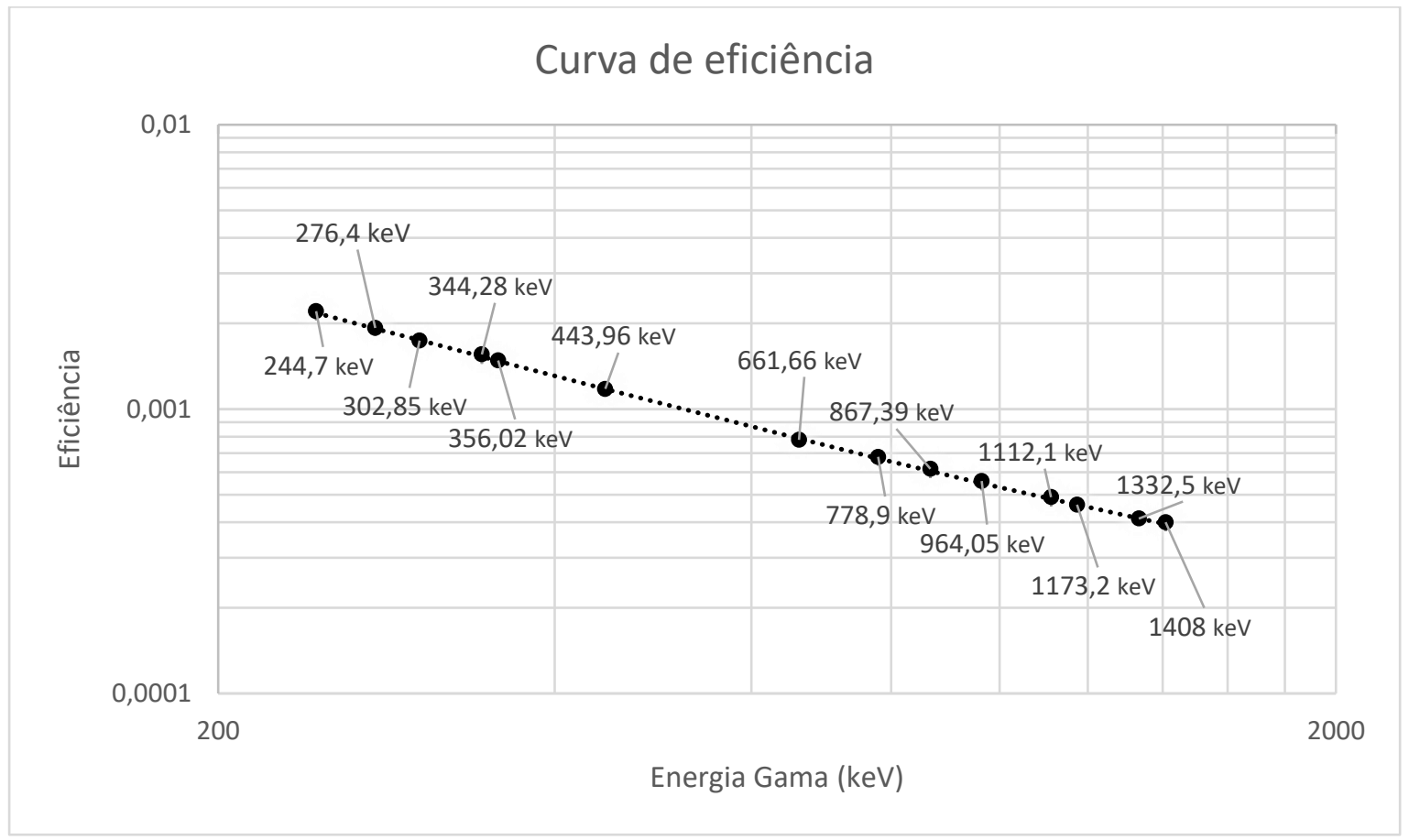

FIGURA 5 - Curva de eficiência, em escala log-log, para o espectrômetro de HPGe em função da energia gama, obtida pelas fontes padrões fornecidas pela IUPAC.

Na TAB. 9 são apresentados os valores das eficiências das amostras de interesse, adquiridos pela curva obtida; os valores em parênteses representam as incertezas nas casas decimais:

TABELA 9 - Valores das eficiências das reações.

\begin{tabular}{ccc}
\hline & Eficiências $(2008$ e 2010) & \\
\hline Produto & $\boldsymbol{E}_{\gamma}(\mathbf{k e V})$ & Eficiência \\
\hline${ }^{198} \mathrm{Au}$ & 411,8 & $1,271(7) \times 10^{-03}$ \\
${ }^{95} \mathrm{Zr}$ & 724,2 & $7,21(5) \times 10^{-04}$ \\
& 756,7 & $6,93(5) \times 10^{-04}$ \\
\hline${ }^{65} \mathrm{Zn}$ & 1115,5 & $4,900(21) \times 10^{-04}$ \\
${ }^{69 \mathrm{~m}} \mathrm{Zn}$ & 438,6 & $1,188(7) \times 10^{-03}$ \\
${ }^{46} \mathrm{Sc}$ & 889,3 & $6,01(4) \times 10^{-04}$ \\
& 1120,5 & $4,897(21) \times 10^{-04}$ \\
\hline & 328,7 & $1,610(9) \times 10^{-03}$ \\
${ }^{140} \mathrm{La}$ & 487,0 & $1,064(6) \times 10^{-03}$ \\
& 815,8 & $6,48(4) \times 10^{-04}$ \\
& 1596,2 & $3,34(4) \times 10^{-04}$ \\
\hline${ }^{60} \mathrm{Co}$ & 1173,2 & $4,671(19) \times 10^{-04}$ \\
& 1332,5 & $4,109(23) \times 10^{-04}$ \\
\hline
\end{tabular}




\subsection{Resultados do código COVAR v4.1}

O exemplo de um resultado adquirido é apresentado na FIG. 6.

\begin{tabular}{|c|c|c|c|}
\hline $\mathrm{N}$ & 14 & & \\
\hline ASP1 & 285037,400000 & ASP2 & 28053,740000 \\
\hline ASP3 & $6,780285 E+09$ & ASP4 & $1,688760 E+09$ \\
\hline $\mathrm{FCDI}(\mathrm{N})$ & $9,968000 \mathrm{E}-01$ & $\operatorname{FCDC}(\mathrm{N})$ & $9,999000 \mathrm{E}-01$ \\
\hline Gthc $(N)$ & $9,999700 \mathrm{E}-01$ & Gthi(N) & $9,999500 \mathrm{E}-01$ \\
\hline$E f c(N)$ & $1,270000 E-03$ & $\operatorname{Efi}(\mathrm{N})$ & $7,210000 \mathrm{E}-04$ \\
\hline $\mathrm{K} \theta(\mathrm{N})$ & $8,887852 E-05$ & $\mathrm{Q} \theta \mathrm{I}(\mathrm{N})$ & 5,652429 \\
\hline $\mathrm{K} 02(\mathrm{~N})$ & $8,887852 E-05$ & & \\
\hline $\mathrm{K} 03(\mathrm{~N})$ & $8,932607 E-05$ & $\mathrm{FII}(\mathrm{N})$ & 49,700000 \\
\hline $\operatorname{Gec}(N)$ & $9,808000 E-01$ & QOII $(N)$ & 5,310000 \\
\hline Q0alfac & 15,737090 & Gei(N) & $9,090200 \mathrm{E}-01$ \\
\hline $\mathrm{Q} \theta \mathrm{Ia}(\mathrm{N})$ & 5,607568 & $\mathrm{Q} 02(\mathrm{~N})$ & 5,603192 \\
\hline RCDI & 10,160400 & $\mathrm{RCDC}$ & 4,014950 \\
\hline $\mathrm{FC}$ & 46,530760 & $\mathrm{FI}$ & 49.672301 \\
\hline $\mathrm{N}$ & 15 & & \\
\hline ASP1 & 341021,400000 & ASP2 & 33401,800000 \\
\hline ASP3 & $6,780285 E+09$ & ASP4 & $1,688760 E+09$ \\
\hline $\mathrm{FCDI}(\mathrm{N})$ & $9,968000 \mathrm{E}-01$ & $\operatorname{FCDC}(\mathrm{N})$ & $9,999000 \mathrm{E}-01$ \\
\hline Gthc $(N)$ & $9,999700 \mathrm{E}-01$ & Gthi(N) & $9,999500 \mathrm{E}-01$ \\
\hline $\operatorname{Efc}(N)$ & $1,270000 E-03$ & $\operatorname{Efi}(\mathrm{N})$ & $6,930000 E-04$ \\
\hline $\mathrm{K} \theta(\mathrm{N})$ & $1,106900 \mathrm{E}-04$ & $\mathrm{Q} \theta \mathrm{I}(\mathrm{N})$ & 5,622156 \\
\hline $\mathrm{K} 02(\mathrm{~N})$ & $1,106900 E-04$ & & \\
\hline $\mathrm{K} 03(\mathrm{~N})$ & $1,111886 \mathrm{E}-04$ & $\mathrm{FII}(\mathrm{N})$ & 49,700000 \\
\hline $\operatorname{Gec}(N)$ & $9,808000 \mathrm{E}-01$ & QOII $(N)$ & 5,310000 \\
\hline Q0alfac & 15,737090 & Gei(N) & $9,090200 \mathrm{E}-01$ \\
\hline QOIa(N) & 5,577559 & $Q 02(N)$ & 5,573184 \\
\hline RCDI & 10,209670 & $\mathrm{RCDC}$ & 4,014950 \\
\hline $\mathrm{FC}$ & 46,530760 & $\mathrm{FI}$ & 49.672301 \\
\hline
\end{tabular}

FIGURA 6 - Exemplo do resultado obtido na primeira execução do COVAR v4.1. Os números de $\mathrm{N}(14$ e 15) referem-se aos índices atribuídos às transições gama de $724,2 \mathrm{keV}$ e 756,7 keV, da reação ${ }^{94} \mathrm{Zr}(\mathrm{n}, \gamma){ }^{95} \mathrm{Zr}$.

As informações apresentadas na FIG. 6 referem-se às transições gama de 724,2 keV e 756,7 keV da reação ${ }^{94} \mathrm{Zr}(\mathrm{n}, \gamma)^{95} \mathrm{Zr}$. Os valores indicados em $\mathrm{KO}(\mathrm{N})$ são os parâmetros $k_{0}$ que foram obtidos pela $\mathrm{EQ}$. (3.1), K02(N) e $\mathrm{K} 03(\mathrm{~N})$ foram obtidos pela EQ. (3.3), enquanto que, para o primeiro, os parâmetros $f$ e $Q_{0, i}$ são calculados pelo próprio código e, para o segundo, os valores estão como dados de entrada. $O$ valor calculado para $f$ foi de 49,67(4), como indicado em FI com seu valor geral, estando de acordo com os dados adquiridos das irradiações 
utilizadas neste trabalho e também com o valor de entrada, 49,7, indicado no campo Fll.

Os valores de $k_{0}$ para as irradiações realizadas nos anos de 2008 e 2010 estão apresentadas nas TAB. 10 e 11.

TABELA 10 - Resultados de $k_{0}$ gerados por meio do COVAR v4.1 utilizando as EQ. (3.1) (Anterior) e a EQ. (3.3) incluída (Presente Trabalho), para os dados de 2008.

\begin{tabular}{|c|c|c|c|c|}
\hline \multicolumn{5}{|c|}{ Valores de $k_{0}$ para os dados de 2008} \\
\hline Produto & $E_{\gamma}(\mathrm{keV})$ & $k_{0}$ (Anterior) & $k_{0}$ (Presente Trabalho) & $\boldsymbol{k}_{\mathbf{0}}$ (Literatura) $^{[88}$ \\
\hline \multirow{2}{*}{${ }^{95} \mathrm{Zr}$} & 724,2 & $8,89(13) \times 10^{-05}$ & $8,93(14) \times 10^{-05}$ & $8,99(10) \times 10^{-5}$ \\
\hline & 756,7 & $1,110(20) \times 10^{-04}$ & $1,110(20) \times 10^{-04}$ & $1,110(20) \times 10^{-4}$ \\
\hline${ }^{65} \mathrm{Zn}$ & 1115,5 & $5,68(10) \times 10^{-03}$ & $5,66(11) \times 10^{-03}$ & $5,720(20) \times 10^{-3}$ \\
\hline \multirow{4}{*}{${ }^{140} \mathrm{La}$} & 328,7 & $2,827(37) \times 10^{-02}$ & $2,828(44) \times 10^{-02}$ & $2,870(3) \times 10^{-2}$ \\
\hline & 487,0 & $6,38(8) \times 10^{-02}$ & $6,39(10) \times 10^{-02}$ & $6,37(6) \times 10^{-2}$ \\
\hline & 815,8 & $3,28(4) \times 10^{-02}$ & $3,27(5) \times 10^{-02}$ & $3,320(20) \times 10^{-2}$ \\
\hline & 1596,2 & $1,37(4) \times 10^{-01}$ & $1,38(7) \times 10^{-01}$ & $1,340(10) \times 10^{-1}$ \\
\hline \multirow{2}{*}{${ }^{46} \mathrm{Sc}$} & 889,0 & $1,197(23)$ & $1,198(25)$ & $1,220(5)$ \\
\hline & 1120,0 & $1,192(22)$ & $1,193(25)$ & $1,220(13)$ \\
\hline
\end{tabular}

TABELA 11 - Resultados de $k_{0}$ gerados por meio do COVAR v4.1 utilizando as EQ. (3.1) (Anterior) e a EQ. (3.3) incluída (Presente Trabalho), para os dados de 2010.

\begin{tabular}{ccccc}
\hline \multicolumn{5}{c}{ Valores de $\boldsymbol{k}_{\mathbf{0}}$ para os dados de 2010 } \\
Produto & $\boldsymbol{E}_{\boldsymbol{\gamma}}(\mathbf{k e V})$ & $\boldsymbol{k}_{\mathbf{0}}$ (Anterior) & $\boldsymbol{k}_{\mathbf{0}}$ (Presente Trabalho) & $\boldsymbol{k}_{\mathbf{0}}$ (Literatura) ${ }^{[88]}$ \\
\hline${ }^{95} \mathbf{Z r}$ & 724,2 & $8,85(9) \times 10^{-05}$ & $8,88(12) \times 10^{-05}$ & $8,99(10) \times 10^{-5}$ \\
& 756,7 & $1,100(20) \times 10^{-04}$ & $1,100(10) \times 10^{-04}$ & $1,110(20) \times 10^{-4}$ \\
\hline${ }^{65} \mathbf{Z n}$ & 1115,5 & $5,59(8) \times 10^{-03}$ & $5,60(9) \times 10^{-03}$ & $5,720(20) \times 10^{-3}$ \\
\hline${ }^{69} \mathrm{~m} \mathbf{Z n}$ & 438,6 & $3,93(10) \times 10^{-4}$ & $3,94(10) \times 10^{-4}$ & $3,980(20) \times 10^{-4}$ \\
\hline${ }^{60} \mathrm{Co}$ & 1173,2 & $1,328(18)$ & $1,330(21)$ & $1,320(5)$ \\
& 1332,5 & $1,315(19)$ & $1,322(22)$ & $1,320(7)$ \\
& 328,7 & $2,88(3) \times 10^{-02}$ & $2,87(4) \times 10^{-02}$ & $2,87(3) \times 10^{-2}$ \\
& 487,0 & $6,41(6) \times 10^{-02}$ & $6,40(9) \times 10^{-02}$ & $6,37(6) \times 10^{-2}$ \\
${ }^{140} \mathrm{La}$ & 815,8 & $3,35(4) \times 10^{-02}$ & $3,35(5) \times 10^{-02}$ & $3,320(20) \times 10^{-2}$ \\
& 1596,2 & $1,340(20) \times 10^{-01}$ & $1,340(20) \times 10^{-01}$ & $1,340(10) \times 10^{-1}$ \\
\hline${ }^{46} \mathrm{Sc}$ & 889,0 & $1,217(12)$ & $1,218(17)$ & $1,220(5)$ \\
& 1120,0 & $1,223(11)$ & $1,224(17)$ & $1,220(13)$ \\
\hline
\end{tabular}

A TAB. 12 apresenta os resultados de $Q_{0}$ calculados pelo COVAR v4.1 para as irradiações de 2008 e 2010 : 
TABELA 12 - Resultados de $Q_{0}$ gerados por meio do COVAR v4.1, para os dados de 2008 e 2010.

\begin{tabular}{|c|c|c|c|c|}
\hline \multicolumn{5}{|c|}{ Valores de $Q_{0}$ para os dados de 2008 e 2010} \\
\hline Ano & Produto & $E_{\gamma}(\mathrm{keV})$ & $Q_{0}$ & $Q_{0}$ (Literatura) $^{[88]}$ \\
\hline \multirow{8}{*}{2008} & \multirow{2}{*}{${ }^{95} \mathrm{Zr}$} & 724,2 & \multirow{2}{*}{$5,63(15)$} & \multirow{2}{*}{$5,31(17)$} \\
\hline & & 756,7 & & \\
\hline & ${ }^{65} \mathrm{Zn}$ & 1115,5 & $1,78(5)$ & $1,91(9)$ \\
\hline & \multirow{4}{*}{${ }^{140} \mathrm{La}$} & 328,7 & \multirow{4}{*}{$1,26(3)$} & \multirow{4}{*}{$1,24(7)$} \\
\hline & & 487,0 & & \\
\hline & & 815,8 & & \\
\hline & & 1596,2 & & \\
\hline & ${ }^{46} \mathrm{Sc}$ & $\begin{array}{c}889,0 \\
1120,0\end{array}$ & $0,485(14)$ & $0,445(26)$ \\
\hline \multirow{12}{*}{2010} & \multirow{2}{*}{${ }^{95} \mathrm{Zr}$} & 724,2 & \multirow{2}{*}{$5,56(13)$} & \multirow{2}{*}{$5,31(17)$} \\
\hline & & 756,7 & & \\
\hline & ${ }^{65} \mathrm{Zn}$ & 1115,5 & $1,97(4)$ & $1,91(9)$ \\
\hline & ${ }^{69 m} \mathrm{Zn}$ & 438,6 & $2,67(18)$ & $2,56(4)$ \\
\hline & \multirow{2}{*}{${ }^{60} \mathrm{Co}$} & 1173,2 & \multirow{2}{*}{$2,17(6)$} & \multirow{2}{*}{$1.99(6)$} \\
\hline & & 1332,5 & & \\
\hline & \multirow{4}{*}{${ }^{140} \mathrm{La}$} & 328,7 & \multirow{4}{*}{$1,22(3)$} & \multirow{4}{*}{$1,24(7)$} \\
\hline & & 487,0 & & \\
\hline & & 815,8 & & \\
\hline & & 1596,2 & & \\
\hline & \multirow[t]{2}{*}{${ }^{46} \mathrm{Sc}$} & 889,0 & \multirow{2}{*}{$0,497(17)$} & \multirow{2}{*}{$0,445(26)$} \\
\hline & & 1120,0 & & \\
\hline
\end{tabular}

Não foram adicionados os valores de K02, que estão indicados na FIG. 6 pois sua criação possui a função de teste para verificar se o cálculo utilizado para o método anterior está correto, em razão de K02 ser uma extensão do mesmo. Por este motivo não foram criadas as derivadas parciais deste campo (K02) para calcular suas incertezas.

Pode-se observar pelas TAB. 10, 11 e 12 que os valores de $k_{0}$ calculados com o método anterior (indicado no campo K0 da FIG. 6) são ligeiramente maiores que os valores de $k_{0}$ do presente trabalho (indicado no campo K03) quando o parâmetro $Q_{0}$, calculado pelo código COVAR v4.1, é menor que $\circ Q_{0}$ da literatura utilizado como referência (usado também como dado de entrada para o cálculo de K03), e menores quando o valor do $Q_{0}$ calculado é superior, e isso está de acordo com as matrizes de correlação entre $k_{0}$ e $Q_{0}$, apresentadas no APÊNDICE $C$ e $D$ deste trabalho. $O$ motivo das matrizes 
estarem no APÊNDICE é por contemplarem todos os alvos utilizados, ou seja, 21 dados (excluindo $\mathrm{Au}$ ), sendo assim uma extensa matriz quadrada com 42 linhas. Essa inversão de proporcionalidade entre os fatores $k_{0}$ e $Q_{0}$ pode ser explicada em razão do componente comparador que, para a determinação do $k_{0}$, aparece no denominador da fração, enquanto na determinação do $Q_{0}$ aparece no numerador. Desta forma o aumento do comparador tende a diminuir o valor do $k_{0}$, enquanto aumenta o valor do $Q_{0}$. É compreensível, portanto, com base nesta afirmação, que o aumento do valor de $Q_{0}$ tende a diminuir o valor de $k_{0}$ e viceversa.

A introdução do parâmetro $Q_{0}$ e $f$ como dados de entrada adicionais podem ter contribuído para a uma flutuação nos resultados entre os resultados com o método anterior e o novo, principalmente $Q_{0}$, visto que os valores calculados e os inseridos como dados de entrada possuem diferenças que podem causar uma ligeira variação no valor final. Os resultados apresentados nas TAB. 10 e 11 indicam que a inclusão da nova EQ. (3.3) acarreta um aumento na incerteza total no valor de $k_{0}$, e isso se explica considerando a inclusão das incertezas dos novos parâmetros adicionados.

Os valores de $k_{0}$ com o método proposto no presente trabalho se comportaram próximos do valor de $k_{0}$ com o método anterior, podendo ser considerados estatisticamente iguais. As matrizes de correlação apresentadas nos APÊNDICES C e D se mostraram coerentes com o esperado. As correlações entre $k_{0}$, tanto para o método novo quanto para o anterior, foram maiores para as correlações de isótopos iguais e menores para correlações de isótopos diferentes. Isso ocorre em virtude dos parâmetros correlacionados. Quando os isótopos são iguais, mais parâmetros associados são idênticos, o que favorece o aumento da correlação, por outro lado, para o caso dos isótopos serem diferentes, há menos parâmetros idênticos, ou seja, os isótopos podem possuir uma certa independência entre si, havendo, assim, uma correlação menor. No APÊNDICE D, a correlação entre $k_{0}$ e $Q_{0}$ é baixa ou nula, em razão de $Q_{0}$ ser um dado de entrada e não um parâmetro calculado, o que também explica as altas correlações entre $Q_{0}$. Analisando o caso entre os parâmetros $k_{0}$ do método novo, as correlações possuíram uma intensidade moderada e positiva para elementos iguais, e fraca para os elementos diferentes, dentro do que se era esperado. 
Com base nos resultados, não houve qualquer erro no código da inclusão da EQ. (3.3) e, apesar do aumento das incertezas, esta equação se comportou de maneira satisfatória, o que a torna como uma nova alternativa para comparar os resultados de $k_{0}$ pelo COVAR v4.1.

\subsection{Resultados do código AKFIT v2.1}

$\mathrm{Na}$ TAB. 13 são apresentados os resultados obtidos pelo código AKFIT v2.1 para os dados das irradiações dos anos de 2008:

TABELA 13 - Resultados dos dados das irradiações do ano de 2008 analisados separadamente.

\begin{tabular}{|c|c|c|c|c|c|c|}
\hline \multicolumn{7}{|c|}{ Dados 2008 - Valores dos ajustes linear e não-linear } \\
\hline & & & $a$ & $\alpha$ & $\chi^{2}$ & $\chi_{\text {red }}^{2}$ \\
\hline \multicolumn{3}{|c|}{ Aju. Linear e Não-Linear } & $25,14(8)$ & $-0,0016(89)$ & 7,48 & 1,07 \\
\hline Ano & Produto & Energia & \multicolumn{2}{|c|}{$k_{0}$ (Linear e Não-Linear) } & \multicolumn{2}{|c|}{$k_{0}$ (Literatura) ${ }^{[88]}$} \\
\hline \multirow{10}{*}{2008} & ${ }^{198} \mathrm{Au}$ & 411 & & & & \\
\hline & \multirow{2}{*}{${ }^{95} \mathrm{Zr}$} & 724,2 & & $\times 10^{-05}$ & \multicolumn{2}{|c|}{$8,99(10) \times 10^{-5}$} \\
\hline & & 756,7 & & $\times 10^{-04}$ & \multicolumn{2}{|c|}{$1,110(20) \times 10^{-4}$} \\
\hline & ${ }^{65} \mathrm{Zn}$ & 1115,5 & \multicolumn{2}{|c|}{$5,63(9) \times 10^{-03}$} & \multicolumn{2}{|c|}{$5,720(20) \times 10^{-3}$} \\
\hline & \multirow{4}{*}{${ }^{140} \mathrm{La}$} & 328,7 & & $\times 10^{-02}$ & \multicolumn{2}{|c|}{$2,87(3) \times 10^{-2}$} \\
\hline & & 487 & & $\times 10^{-02}$ & \multicolumn{2}{|c|}{$6,37(6) \times 10^{-2}$} \\
\hline & & 815,8 & & $\times 10^{-02}$ & \multicolumn{2}{|c|}{$3,320(20) \times 10^{-2}$} \\
\hline & & 1596,2 & & $\times 10^{-01}$ & \multicolumn{2}{|c|}{$1,340(10) \times 10^{-1}$} \\
\hline & \multirow{2}{*}{${ }^{46} \mathrm{Sc}$} & 889 & & (24) & \multicolumn{2}{|c|}{$1,220(5)$} \\
\hline & & 1120 & & (23) & \multicolumn{2}{|c|}{$1,220(13)$} \\
\hline
\end{tabular}

A execução do AKFIT v2.1 obteve um valor para o parâmetro $\alpha$ de 0,0016 $\pm 0,0089$, valor este compatível com zero e de acordo com as referências Dias et. $A /{ }^{[9]}$. Os valores de $k_{0}$ para os dois métodos de ajuste foram idênticos, o que era esperado, visto que o ajuste não-linear é um caso geral do ajuste linear. Isso também indicou que o algoritmo foi executado corretamente, com ambos os métodos chegando aos mesmos resultados. O chi-quadrado com valor próximo de 1 indica que o modelo de ajuste foi realizado satisfatoriamente.

A TAB. 14 a seguir apresenta os resultados de $k_{0}$ para os dados irradiados no ano de 2010: 
TABELA 14 - Resultados dos dados das irradiações do ano de 2010 analisados separadamente.

\begin{tabular}{|c|c|c|c|c|c|c|}
\hline \multicolumn{7}{|c|}{ Dados 2010 - Valores dos ajustes linear e não-linear } \\
\hline & & & $a$ & $\alpha$ & $\chi^{2}$ & $\chi_{\text {red }}^{2}$ \\
\hline \multicolumn{3}{|c|}{ Aju. Linear e Não-Linear } & $25,54(6)$ & $0,0035(67)$ & 11,41 & 1,04 \\
\hline \multirow[t]{2}{*}{ Ano } & \multicolumn{2}{|c|}{ Produto Energia } & \multicolumn{2}{|c|}{$k_{0}$ (Linear e Não-Linear) } & \multicolumn{2}{|c|}{$\boldsymbol{k}_{\mathbf{0}}$ (Literatura) ${ }^{[88]}$} \\
\hline & ${ }^{198} \mathrm{Au}$ & 411 & & & & \\
\hline \multirow{12}{*}{2010} & \multirow{2}{*}{${ }^{95} \mathrm{Zr}$} & 724,2 & \multicolumn{2}{|c|}{$8,99(9) \times 10^{-05}$} & \multicolumn{2}{|c|}{$8,99(10) \times 10^{-5}$} \\
\hline & & 756,7 & \multicolumn{2}{|c|}{$1,120(10) \times 10^{-04}$} & \multicolumn{2}{|c|}{$1,110(20) \times 10^{-4}$} \\
\hline & ${ }^{65} \mathrm{Zn}$ & 1115,5 & \multicolumn{2}{|c|}{$5,59(5) \times 10^{-03}$} & \multicolumn{2}{|c|}{$5,720(20) \times 10^{-3}$} \\
\hline & $69 \mathrm{~m} \mathrm{Zn}$ & 438,6 & \multicolumn{2}{|c|}{$3,91(9) \times 10^{-04}$} & \multicolumn{2}{|c|}{$3,980(20) \times 10^{-4}$} \\
\hline & \multirow{4}{*}{${ }^{140} \mathrm{La}$} & 328,7 & & $\times 10^{-02}$ & \multicolumn{2}{|c|}{$2,87(3) \times 10^{-2}$} \\
\hline & & 487 & & $\times 10^{-02}$ & \multicolumn{2}{|c|}{$6,37(6) \times 10^{-2}$} \\
\hline & & 815,8 & & $\times 10^{-02}$ & \multicolumn{2}{|c|}{$3,320(20) \times 10^{-2}$} \\
\hline & & 1596,2 & & $\times 10^{-01}$ & \multicolumn{2}{|c|}{$1,340(10) \times 10^{-1}$} \\
\hline & \multirow{2}{*}{${ }^{60} \mathrm{Co}$} & 1173,2 & & (16) & \multicolumn{2}{|c|}{$1,320(5)$} \\
\hline & & 1332,5 & & (18) & \multicolumn{2}{|c|}{$1,320(7)$} \\
\hline & \multirow{2}{*}{${ }^{46} \mathrm{Sc}$} & 889 & & (13) & \multicolumn{2}{|c|}{$1,220(5)$} \\
\hline & & 1120 & & (12) & \multicolumn{2}{|c|}{$1,220(13)$} \\
\hline
\end{tabular}

O modelo, para este caso, também se mostrou bem ajustado, como indicado pelo chi-quadrado reduzido. O valor de $\alpha$ foi de $0,0035 \pm 0,0067$. As diferenças entre os valores de $k_{0}$ ajustado e a literatura foram baixas. As TAB. 15 e 16 apresentam os resultados para os dados de 2008 e 2010 ajustados simultaneamente.

TABELA 15 - Comparação dos valores de $a, \alpha, \chi^{2}$ e $\chi_{\text {reduzido }}^{2}$ entre os ajustes linear e não-linear obtidos pelo código AKFIT v2.1.

\begin{tabular}{cccccc}
\hline \multicolumn{5}{c}{ Valores dos ajustes linear e não-linear } \\
\hline & $\boldsymbol{a}$ & $\boldsymbol{\alpha}$ & $\chi^{2}$ & $\chi_{\text {red }}^{2}$ \\
\hline Aju. Linear e Não-Linear & $25,41(5)$ & $0,007(8)$ & 22,2 & 1,11 \\
\hline
\end{tabular}


TABELA 16 - Comparação dos valores $k_{0}$ entre os ajustes linear e não-linear obtidos pelo código AKFIT v2.1.

\begin{tabular}{|c|c|c|c|c|}
\hline \multicolumn{5}{|c|}{ Dados 2008 e 2010 - Valores de $k_{0}$ para os dados de 2008 e 2010} \\
\hline Ano & Produto & Energia & $k_{0}$ (Linear e Não-Linear) & $k_{0}$ (Literatura) $^{[88]}$ \\
\hline \multirow{10}{*}{2008} & ${ }^{198} \mathrm{Au}$ & 411 & 1 & 1 \\
\hline & \multirow{2}{*}{${ }^{95} \mathrm{Zr}$} & 724,2 & $8,96(13) \times 10^{-05}$ & $8,99(10) \times 10^{-5}$ \\
\hline & & 756,7 & $1,120(20) \times 10^{-04}$ & $1,110(20) \times 10^{-4}$ \\
\hline & ${ }^{65} \mathrm{Zn}$ & 1115,5 & $5,65(8) \times 10^{-03}$ & $5,720(20) \times 10^{-3}$ \\
\hline & \multirow{4}{*}{${ }^{140} \mathrm{La}$} & 328,7 & $2,84(5) \times 10^{-02}$ & $2,87(3) \times 10^{-2}$ \\
\hline & & 487 & $6,32(10) \times 10^{-02}$ & $6,37(6) \times 10^{-2}$ \\
\hline & & 815,8 & $3,24(6) \times 10^{-02}$ & $3,320(20) \times 10^{-2}$ \\
\hline & & 1596,2 & $1,36(3) \times 10^{-01}$ & $1,340(10) \times 10^{-1}$ \\
\hline & \multirow{2}{*}{${ }^{46} \mathrm{Sc}$} & 889 & $1,186(24)$ & $1,220(5)$ \\
\hline & & 1120 & $1,185(23)$ & $1,220(13)$ \\
\hline \multirow{13}{*}{2010} & ${ }^{198} \mathrm{Au}$ & 411 & 1 & 1 \\
\hline & \multirow{2}{*}{${ }^{95} \mathrm{Zr}$} & 724,2 & $8,89(9) \times 10^{-05}$ & $8,99(10) \times 10^{-5}$ \\
\hline & & 756,7 & $1,100(10) \times 10^{-04}$ & $1,110(20) \times 10^{-4}$ \\
\hline & ${ }^{65} \mathrm{Zn}$ & 1115,5 & $5,58(5) \times 10^{-03}$ & $5,720(20) \times 10^{-3}$ \\
\hline & $69 \mathrm{~m} \mathrm{Zn}$ & 438,6 & $3,93(9) \times 10^{-04}$ & $3,980(20) \times 10^{-4}$ \\
\hline & \multirow{4}{*}{${ }^{140} \mathrm{La}$} & 328,7 & $2,89(3) \times 10^{-02}$ & $2,87(3) \times 10^{-2}$ \\
\hline & & 487 & $6,42(6) \times 10^{-02}$ & $6,37(6) \times 10^{-2}$ \\
\hline & & 815,8 & $3,33(3) \times 10^{-02}$ & $3,320(20) \times 10^{-2}$ \\
\hline & & 1596,2 & $1,38(3) \times 10^{-01}$ & $1,340(10) \times 10^{-1}$ \\
\hline & \multirow{2}{*}{${ }^{60} \mathrm{Co}$} & 1173,2 & $1,344(16)$ & $1,320(5)$ \\
\hline & & 1332,5 & $1,320(17)$ & $1,320(7)$ \\
\hline & \multirow{2}{*}{${ }^{46} \mathrm{Sc}$} & 889 & $1,237(13)$ & $1,220(5)$ \\
\hline & & 1120 & $1,225(12)$ & $1,220(13)$ \\
\hline
\end{tabular}

O modelo ajustado possuiu um chi-quadrado reduzido dentro do limite aceitável, sugerindo um bom ajuste. O valor de $\alpha$ foi de $0,007 \pm 0,008$. Os valores de $k_{0}$ foram semelhantes aos resultados obtidos quando analisando os dados de 2008 e 2010 separadamente.

As flutuações nos resultados obtidos para este caso estão relacionadas com os pontos de cada alvo de cada período de irradiação. A curva do modelo ajustado irá compreender todos os pontos e, para isso, poderá ter inclinações que irá afastá-la ou aproximá-la do valor referencial para obter o melhor resultado, tentando minimizar a soma dos quadrados das diferenças entre os valores estimados e todos os dados observados. Estas flutuações também podem ser notadas pelos gráficos dos resíduos dos dados de 2008, apresentados nas FIG. 10 e 12. 
As FIG. 7 e 8 apresentam a comparação dos valores de $k_{0}$ dos dados de 2008 e 2010 analisados de modo separado:

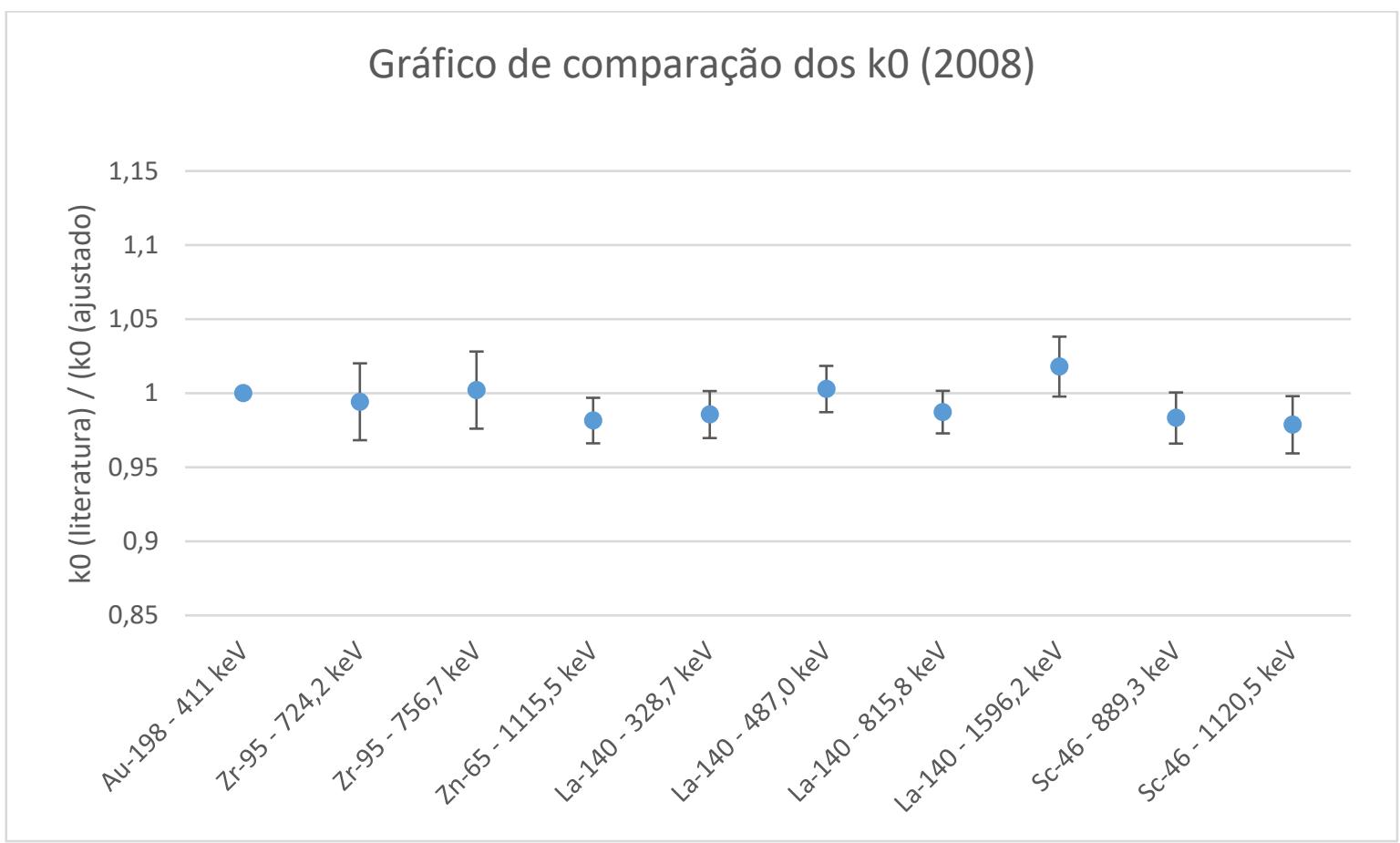

FIGURA 7 - Resultados de $k_{0}$ para os dados de 2008 analisado pelo AKFIT v2.1. Quanto mais próximo de 1, melhor é o resultado.

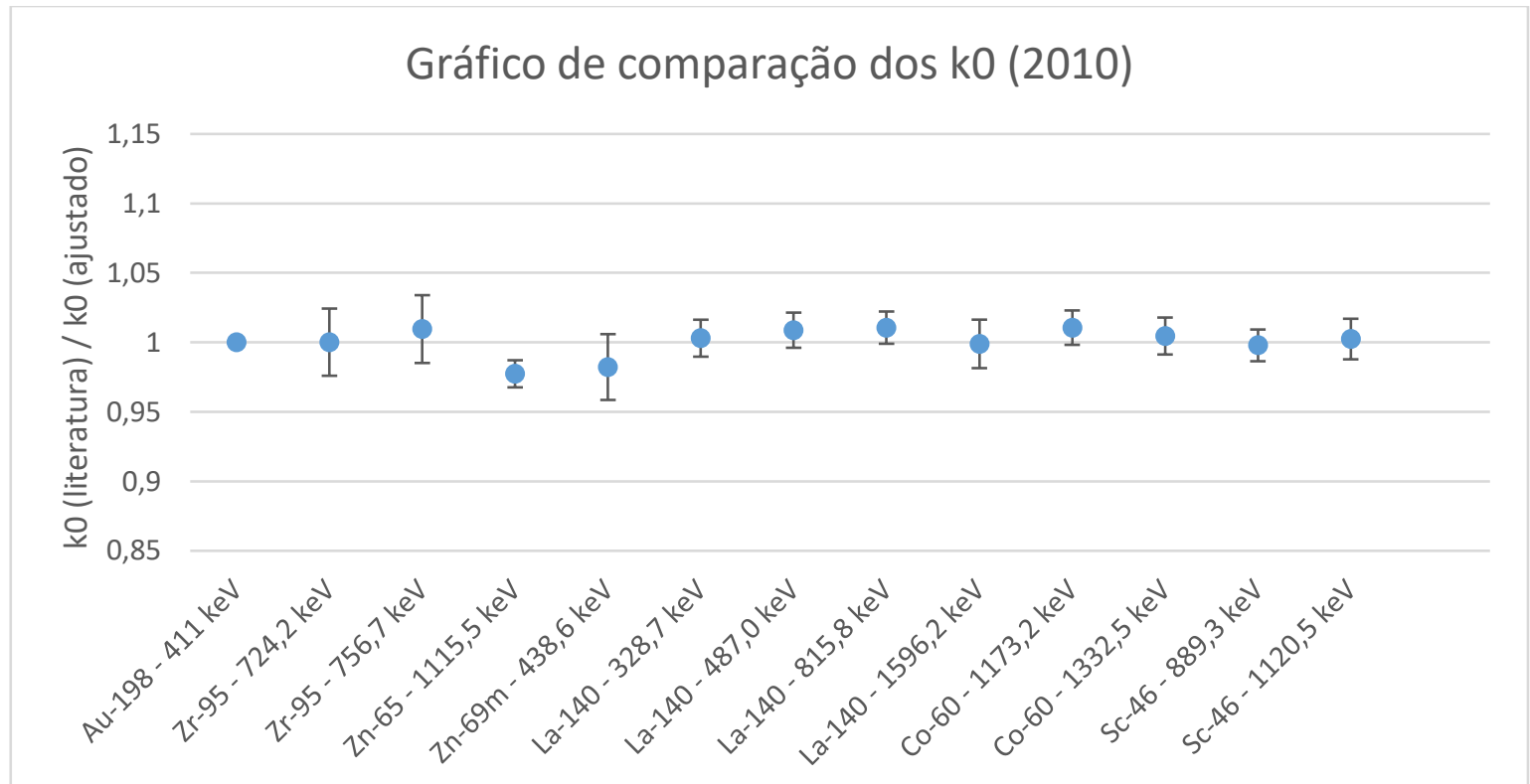

FIGURA 8 - Resultados de $k_{0}$ para os dados de 2010 analisado pelo AKFIT v2.1. Quanto mais próximo de 1, melhor é o resultado. 
A FIG. 9 apresenta a comparação dos valores de $k_{0}$ dos dados de 2008 e 2010 analisados simultaneamente:

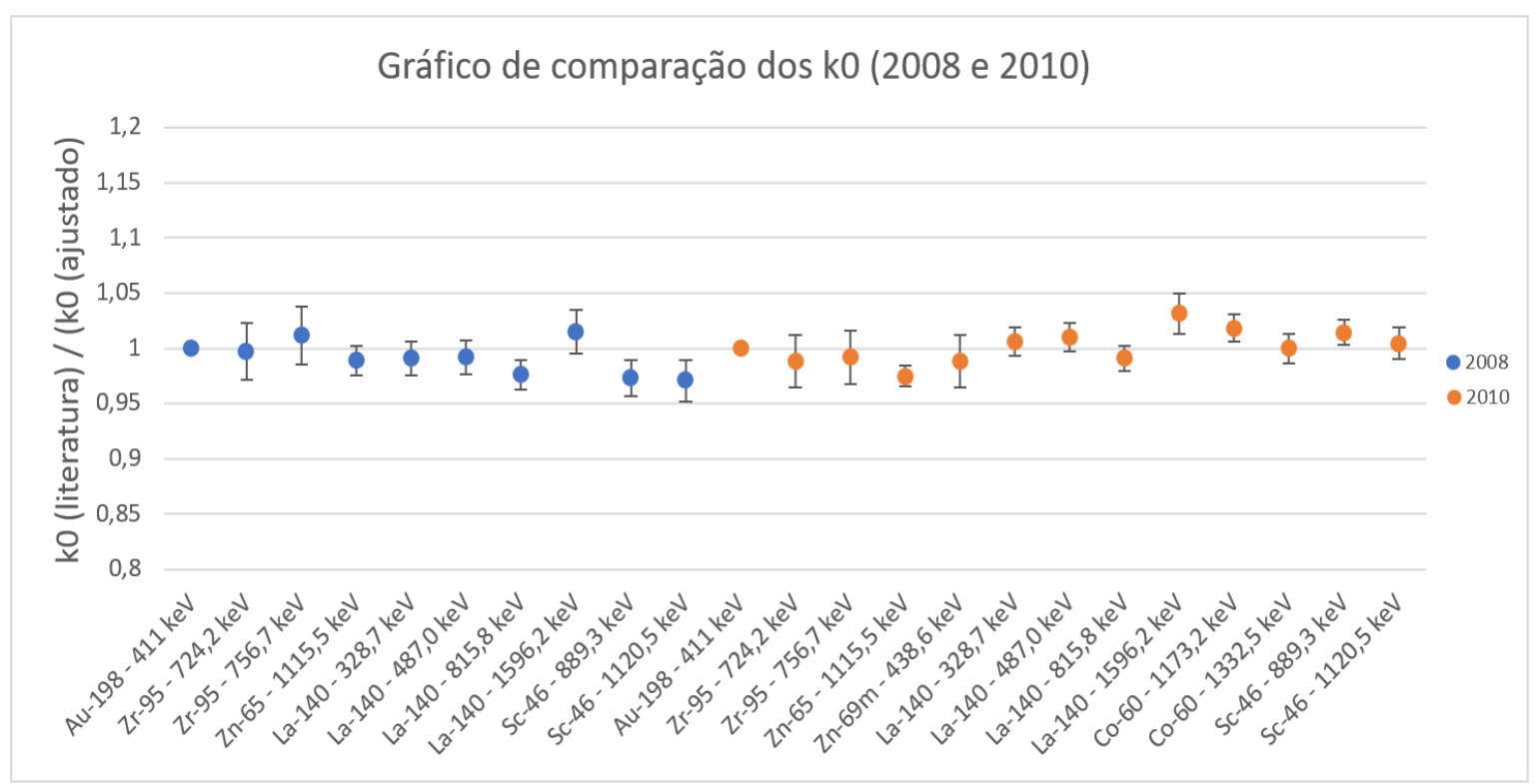

FIGURA 9 - Resultados de $k_{0}$ para os dados de 2008 e 2010 analisados simultaneamente pelo AKFIT v2.1. Quanto mais próximo de 1, melhor é o resultado.

Pode-se observar que os valores de $k_{0}$ apresentaram comportamentos semelhantes para as situações nos quais os dados foram ajustados individualmente e em conjunto. Pelas FIG. 8 e 9 também é possível observar que os alvos tenderam à uma reta, próxima da unidade, sem pontos que se afastam significativamente.

Os resíduos dos ajustes estão apresentados nas FIG. 10, 11 e 12. Foi utilizado índices na FIG. 12 para representar os todos os alvos utilizados e assim ter espaço suficiente para todas as legendas. A representação de cada índice está apresentada na TAB. 17. 


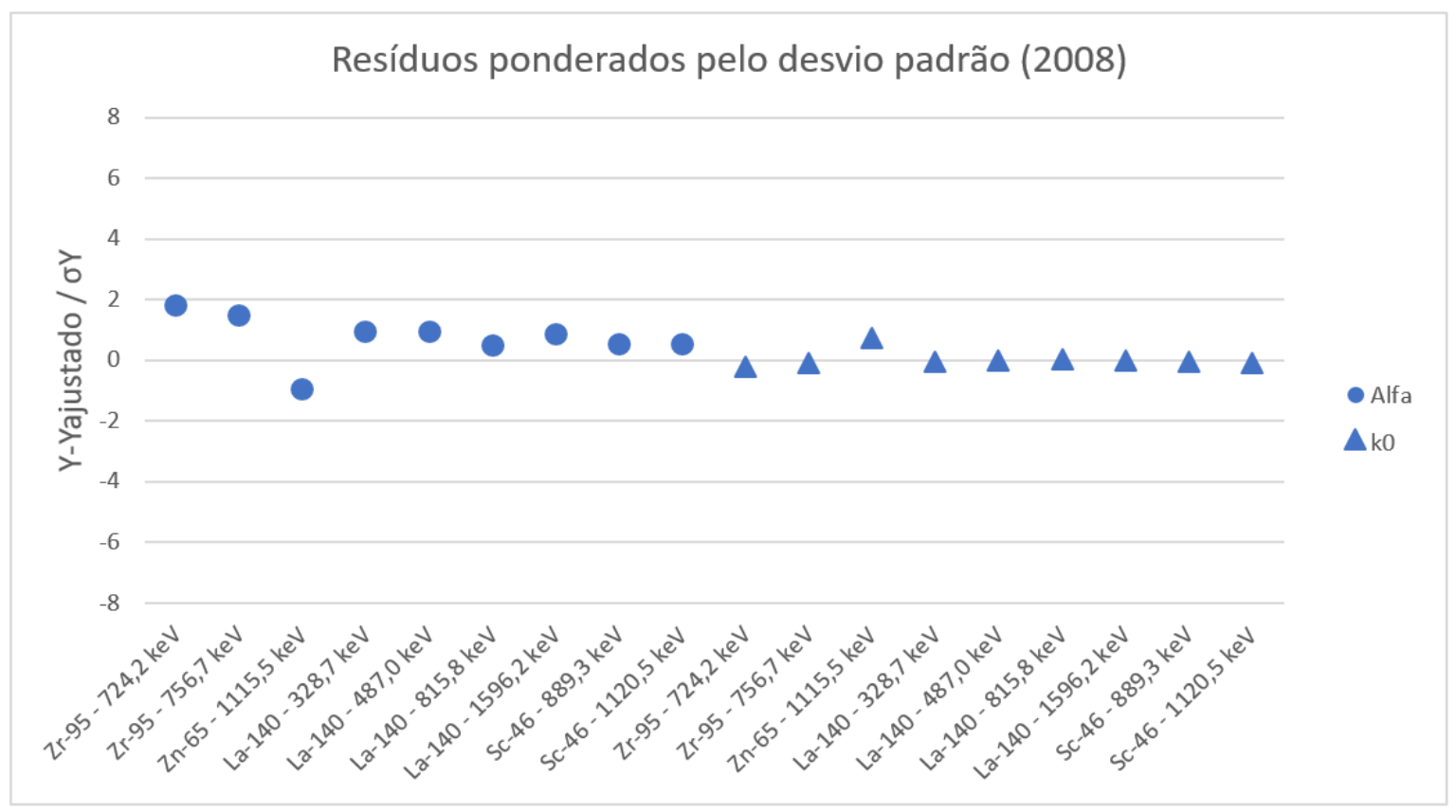

FIGURA 10 - Resíduos normalizados do ajuste dos dados de 2008 analisados separadamente.

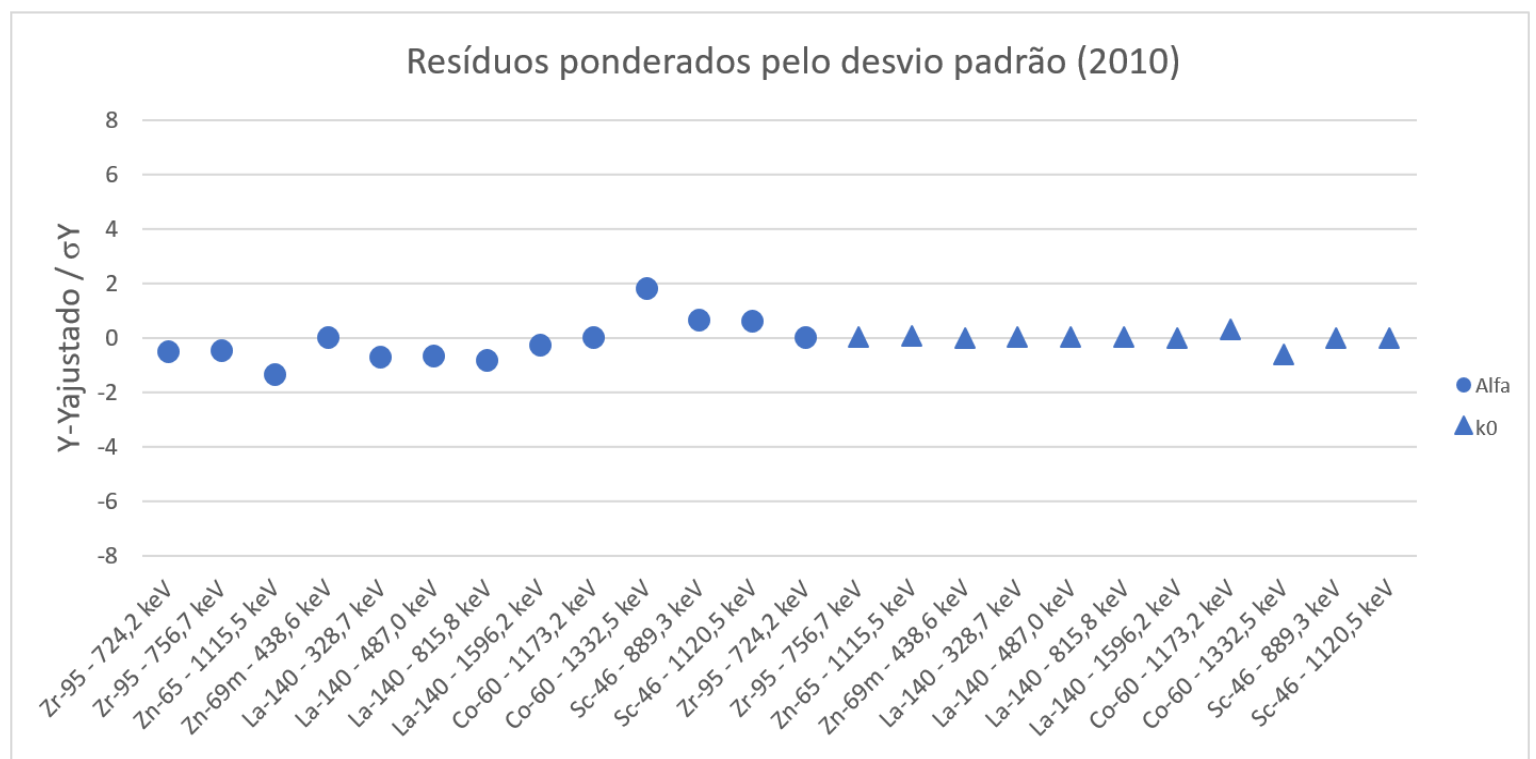

FIGURA 11 - Resíduos normalizados do ajuste dos dados de 2010 analisados separadamente. 


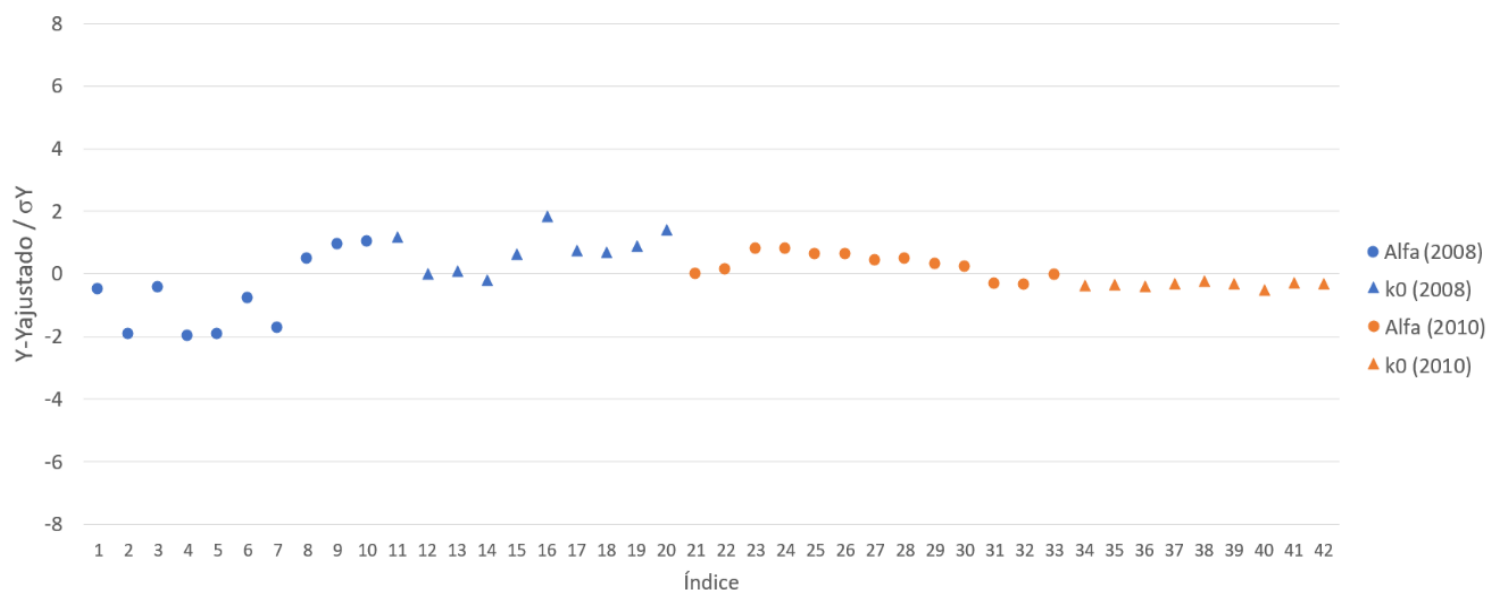

FIGURA 12 - Resíduos normalizados do ajuste com ambos os dados de 2008 e 2010 analisados simultaneamente.

TABELA 17 - Representação de cada índice da FIG. 11, com os alvos e suas respectivas transições gama.

\begin{tabular}{llllllllllll}
\hline Índice & Prod. & $\begin{array}{c}\text { Energia } \\
(\mathbf{k e V})\end{array}$ & Índice & Prod. & $\begin{array}{c}\text { Energia } \\
\text { (keV) }\end{array}$ & Índice & Prod. & $\begin{array}{c}\text { Energia } \\
\text { (keV) }\end{array}$ & Índice & $\begin{array}{c}\text { Prod. } \\
\text { Energia } \\
\text { (keV) }\end{array}$ \\
\hline $\mathbf{1}$ & ${ }^{95} \mathrm{Zr}$ & 724,2 & $\mathbf{1 2}$ & ${ }^{65} \mathrm{Zn}$ & 1115,5 & $\mathbf{2 3}$ & ${ }^{140} \mathrm{La}$ & 328,7 & $\mathbf{3 4}$ & ${ }^{69 m \mathrm{Zn}}$ & 438,6 \\
$\mathbf{2}$ & ${ }^{95} \mathrm{Zr}$ & 756,7 & $\mathbf{1 3}$ & ${ }^{140} \mathrm{La}$ & 328,7 & $\mathbf{2 4}$ & ${ }^{140} \mathrm{La}$ & 487,0 & $\mathbf{3 5}$ & ${ }^{140} \mathrm{La}$ & 328,7 \\
$\mathbf{3}$ & ${ }^{65} \mathrm{Zn}$ & 1115,5 & $\mathbf{1 4}$ & ${ }^{140} \mathrm{La}$ & 487,0 & $\mathbf{2 5}$ & ${ }^{140} \mathrm{La}$ & 815,8 & $\mathbf{3 6}$ & ${ }^{140} \mathrm{La}$ & 487,0 \\
\hline $\mathbf{4}$ & ${ }^{140} \mathrm{La}$ & 328,7 & $\mathbf{1 5}$ & ${ }^{140} \mathrm{La}$ & 815,8 & $\mathbf{2 6}$ & ${ }^{140} \mathrm{La}$ & 1596,5 & $\mathbf{3 7}$ & ${ }^{140} \mathrm{La}$ & 815,8 \\
\hline $\mathbf{5}$ & ${ }^{140} \mathrm{La}$ & 487,0 & $\mathbf{1 6}$ & ${ }^{140} \mathrm{La}$ & 1596,5 & $\mathbf{2 7}$ & ${ }^{60} \mathrm{Co}$ & 1173,2 & $\mathbf{3 8}$ & ${ }^{140} \mathrm{La}$ & 1596,5 \\
\hline $\mathbf{6}$ & ${ }^{140} \mathrm{La}$ & 815,8 & $\mathbf{1 7}$ & ${ }^{46} \mathrm{Sc}$ & 889,3 & $\mathbf{2 8}$ & ${ }^{60} \mathrm{Co}$ & 1132,5 & $\mathbf{3 9}$ & ${ }^{60} \mathrm{Co}$ & 1173,2 \\
\hline $\mathbf{7}$ & ${ }^{140} \mathrm{La}$ & 1596,5 & $\mathbf{1 8}$ & ${ }^{46} \mathrm{Sc}$ & 1120,5 & $\mathbf{2 9}$ & ${ }^{46} \mathrm{Sc}$ & 889,3 & $\mathbf{4 0}$ & ${ }^{60} \mathrm{Co}$ & 1132,5 \\
\hline $\mathbf{8}$ & ${ }^{46} \mathrm{Sc}$ & 889,3 & $\mathbf{1 9}$ & ${ }^{95} \mathrm{Zr}$ & 724,2 & $\mathbf{3 0}$ & ${ }^{46} \mathrm{Sc}$ & 1120,5 & $\mathbf{4 1}$ & ${ }^{46} \mathrm{Sc}$ & 889,3 \\
\hline $\mathbf{9}$ & ${ }^{46} \mathrm{Sc}$ & 1120,5 & $\mathbf{2 0}$ & ${ }^{95} \mathrm{Zr}$ & 756,7 & $\mathbf{3 1}$ & ${ }^{95} \mathrm{Zr}$ & 724,2 & $\mathbf{4 2}$ & ${ }^{46} \mathrm{Sc}$ & 1120,5 \\
\hline $\mathbf{1 0}$ & ${ }^{95} \mathrm{Zr}$ & 724,2 & $\mathbf{2 1}$ & ${ }^{65} \mathrm{Zn}$ & 1115,5 & $\mathbf{3 2}$ & ${ }^{95} \mathrm{Zr}$ & 756,7 & & & \\
\hline $\mathbf{1 1}$ & ${ }^{95} \mathrm{Zr}$ & 756,7 & $\mathbf{2 2}$ & ${ }^{69 m} \mathrm{Zn}$ & 438,6 & $\mathbf{3 3}$ & ${ }^{65} \mathrm{Zn}$ & 1115,5 & & & \\
\hline
\end{tabular}

Os resíduos das FIG. apresentadas mostraram que os ajustes realizados estiveram dentro do aceitável, com todos os desvios padrão de cada ponto entre o intervalo $(-2,2)$, ou seja, não houve pontos mal ajustados que possam ser considerados como outliers, o que corroboram com as informações das TAB. 13, 14, 15 e 16.

As matrizes de correlação foram iguais entre os ajustes linear e nãolinear, o que era esperado já que são os mesmos dados. Pode-se verificar esta afirmação ao analisar o valor de $\lambda$, parâmetro utilizado no método de ajuste nãolinear. Por ser um valor pequeno e, considerando $R^{-1}=V_{A}$, sendo $V_{A}$ a matriz de 
covariância utilizado no método de ajuste linear e a correlação dada por $V_{A(i, j)} /\left(\sigma_{i} * \sigma_{j}\right)$, a matriz $R(\lambda)^{-1}$ se torna a própria matriz $R^{-1}$. Desse modo, os dois métodos de ajuste exibiram os mesmos resultados.

A matriz (APÊNDICE E) exibiu um comportamento de fraca correlação para todos os alvos. Um fator que influenciou em uma flutuação maior da correlação foi o comparador ${ }^{198} \mathrm{Au}$, igual para todos os alvos nos seus respectivos períodos de irradiação. Pode-se observar uma correlação positiva ligeiramente maior para isótopos iguais, porém, não pode ser considerada uma correlação moderada ou forte, diferentemente apresentado pela matriz de correlação gerado pelo COVAR v4.1. Isso se explica pela ausência das correlações dos parâmetros associados às taxas de contagens específicas $A_{S p, i}$ e $\left(A_{S p}\right)_{C d, i}$, como o fator de saturação $S$, da Intensidade média do pico total de energia, $N_{P}$, e massa $w$ das amostras, o que proporcionou menos fatores que influenciassem no aumento da correlação. Para isótopos diferentes, as correlações obtiveram valores positivos e baixos. Em alguns casos, como as correlações entre ${ }^{95} \mathrm{Zr}$, ${ }^{65} \mathrm{Zn}$ e ${ }^{140} \mathrm{La}$ para as irradiações do ano de 2010, apesar de possuírem um valor maior em relação as demais correlações, ainda é um valor que pode ser considerado como uma correlação fraca. As matrizes das correlações dos dados de 2008 e 2010 analisados separadamente podem ser observadas no APÊNDICE F.

A correlação entre os parâmetros $a$ e $\alpha$ foi forte e negativa, o que é previsto considerando que para o cálculo de $\alpha$, dada pela EQ. (3.4), a é o coeficiente linear e $\alpha$ o coeficiente angular, ou seja, no valor de $a$ é onde a reta intercepta o eixo $\mathrm{Y}$ e, consequentemente, influencia diretamente no valor de $\alpha$. De acordo com a equação, quando se eleva o valor de $a$, a inclinação da reta $\alpha$ tende a diminuir para que se mantenha o valor de Y. Quando diminui o valor de $a$, o valor de $\alpha$ tende a aumentar para que se mantenha o resultado.

A correlação entre $k_{0}$ e $\alpha$ foi fraca, indicando uma independência entre os parâmetros, o que é justificável em razão de a ser um parâmetro de ajuste para $Q_{0}$, não exercendo, portanto, uma dependência sobre o fator $k_{0}$. As correlações de $k_{0}$ entre as irradiações de 2008 e 2010 foram baixas em todos os casos. Dado o fato de serem irradiações independentes, a correlação está de acordo com o previsto. 
Realizou-se também uma comparação entre os valores de $k_{0}$ adquiridos analisando separadamente os dados de 2008 e 2010 pelo código AKFIT v2.1 e os valores de $k_{0}$ do código COVAR v4.1 com o método novo (campo K03 da FIG. 6), conforme apresentado nas TAB. 18 e 19:

TABELA 18 - Comparação entre os valores de $k_{0}$ do código COVAR v4.1 com o método novo e AKFIT v2.1 para os dados de 2008 analisado separadamente.

\begin{tabular}{|c|c|c|c|c|c|}
\hline \multicolumn{6}{|c|}{ Valores de $k_{0}$ para os dados de 2008} \\
\hline Ano & Produto & Energia & $k_{0}$ (COVAR V4.1) & $k_{0}$ (AKFIT v2.1) & $k_{0}$ (Literatura) $^{[88]}$ \\
\hline \multirow{9}{*}{2008} & \multirow{2}{*}{${ }^{95} \mathrm{Zr}$} & 724,2 & $8,93(14) \times 10^{-05}$ & $8,94(14) \times 10^{-05}$ & $8,99(10) \times 10^{-5}$ \\
\hline & & 756,7 & $1,11(2) \times 10^{-04}$ & $1,11(2) \times 10^{-04}$ & $1,11(2) \times 10^{-4}$ \\
\hline & ${ }^{65} \mathrm{Zn}$ & 1115,5 & $5,66(11) \times 10^{-03}$ & $5,63(9) \times 10^{-03}$ & $5,72(2) \times 10^{-3}$ \\
\hline & \multirow{4}{*}{${ }^{140} \mathrm{La}$} & 328,7 & $2,83(4) \times 10^{-02}$ & $2,83(4) \times 10^{-02}$ & $2,87(3) \times 10^{-2}$ \\
\hline & & 487 & $6,39(10) \times 10^{-02}$ & $6,39(9) \times 10^{-02}$ & $6,37(6) \times 10^{-2}$ \\
\hline & & 815,8 & $3,27(5) \times 10^{-02}$ & $3,28(5) \times 10^{-02}$ & $3,32(2) \times 10^{-2}$ \\
\hline & & 1596,2 & $1,38(7) \times 10^{-01}$ & $1,36(2) \times 10^{-01}$ & $1,34(1) \times 10^{-1}$ \\
\hline & \multirow{2}{*}{${ }^{46} \mathrm{Sc}$} & 889 & $1,198(25)$ & $1,199(24)$ & $1,220(5)$ \\
\hline & & 1120 & $1,193(25)$ & $1,195(23)$ & $1,220(13)$ \\
\hline
\end{tabular}

TABELA 19 - Comparação entre os valores de $k_{0}$ do código COVAR v4.1 com o método novo e AKFIT v2.1 para os dados de 2010 analisado separadamente.

\begin{tabular}{|c|c|c|c|c|c|}
\hline \multicolumn{6}{|c|}{ Valores de $k_{0}$ para os dados de 2010} \\
\hline Ano & Produto & Energia & $k_{0}$ (COVAR V4.1) & $k_{0}$ (AKFIT v2.1) & $k_{0}$ (Literatura) ${ }^{[88]}$ \\
\hline \multirow{12}{*}{2010} & \multirow{2}{*}{${ }^{95} \mathrm{Zr}$} & 724,2 & $8,88(12) \times 10^{-05}$ & $8,99(9) \times 10^{-05}$ & $8,99(10) \times 10^{-5}$ \\
\hline & & 756,7 & $1,10(1) \times 10^{-04}$ & $1,12(1) \times 10^{-04}$ & $1,11(2) \times 10^{-4}$ \\
\hline & ${ }^{65} \mathrm{Zn}$ & 1115,5 & $5,60(9) \times 10^{-03}$ & $5,59(5) \times 10^{-03}$ & $5,72(2) \times 10^{-3}$ \\
\hline & $69 \mathrm{~m} \mathbf{Z n}$ & 438,6 & $3,94(10) \times 10^{-4}$ & $3,91(9) \times 10^{-04}$ & $3,98(2) \times 10^{-4}$ \\
\hline & \multirow{4}{*}{${ }^{140} \mathrm{La}$} & 328,7 & $2,87(4) \times 10^{-02}$ & $2,88(3) \times 10^{-02}$ & $2,87(3) \times 10^{-2}$ \\
\hline & & 487 & $6,40(9) \times 10^{-02}$ & $6,42(6) \times 10^{-02}$ & $6,37(6) \times 10^{-2}$ \\
\hline & & 815,8 & $3,35(5) \times 10^{-02}$ & $3,34(3) \times 10^{-02}$ & $3,32(2) \times 10^{-2}$ \\
\hline & & 1596,2 & $1,34(2) \times 10^{-01}$ & $1,34(2) \times 10^{-01}$ & $1,34(1) \times 10^{-1}$ \\
\hline & \multirow{2}{*}{${ }^{60} \mathrm{Co}$} & 1173,2 & $1,330(21)$ & $1,334(16)$ & $1,320(5)$ \\
\hline & & 1332,5 & $1,322(22)$ & $1,326(18)$ & $1,320(7)$ \\
\hline & \multirow{2}{*}{${ }^{46} \mathrm{Sc}$} & 889 & $1,218(17)$ & $1,217(13)$ & $1,220(5)$ \\
\hline & & 1120 & $1,224(17)$ & $1,223(12)$ & $1,220(13)$ \\
\hline
\end{tabular}

Na TAB. 20 é apresentado a comparação dos $k_{0}$ com o método novo obtido pelo COVAR v4.1 com o obtido pelo AKFIT v2.1 analisando o ajuste simultâneo dos dados de 2008 e 2010: 
TABELA 20 - Comparação entre os valores de $k_{0}$ do código AKFIT v2.1 com ajuste simultâneo dos dados e do código COVAR v4.1 com o método novo.

\begin{tabular}{|c|c|c|c|c|c|}
\hline \multicolumn{6}{|c|}{ Valores de $k_{0}$ para os dados de 2008 e 2010} \\
\hline Ano & Produto & Energia & $k_{0}($ COVAR V4.1) & $k_{0}$ (AKFIT v2.1) & $k_{0}$ (Literatura) ${ }^{[88]}$ \\
\hline \multirow{9}{*}{2008} & \multirow{2}{*}{${ }^{95} \mathrm{Zr}$} & 724,2 & $8,93(14) \times 10^{-05}$ & $8,96(13) \times 10^{-05}$ & $8,99(10) \times 10^{-5}$ \\
\hline & & 756,7 & $1,11(2) \times 10^{-04}$ & $1,12(2) \times 10^{-04}$ & $1,11(2) \times 10^{-4}$ \\
\hline & ${ }^{65} \mathrm{Zn}$ & 1115,5 & $5,66(11) \times 10^{-03}$ & $5,65(8) \times 10^{-03}$ & $5,72(2) \times 10^{-3}$ \\
\hline & \multirow{4}{*}{${ }^{140} \mathrm{La}$} & 328,7 & $2,828(44) \times 10^{-02}$ & $2,84(4) \times 10^{-02}$ & $2,87(3) \times 10^{-2}$ \\
\hline & & 487 & $6,39(10) \times 10^{-02}$ & $6,32(9) \times 10^{-02}$ & $6,37(6) \times 10^{-2}$ \\
\hline & & 815,8 & $3,27(5) \times 10^{-02}$ & $3,24(5) \times 10^{-02}$ & $3,32(2) \times 10^{-2}$ \\
\hline & & 1596,2 & $1,38(7) \times 10^{-01}$ & $1,36(2) \times 10^{-01}$ & $1,34(1) \times 10^{-1}$ \\
\hline & \multirow{2}{*}{${ }^{46} \mathrm{Sc}$} & 889 & $1,198(25)$ & $1,186(24)$ & $1,220(5)$ \\
\hline & & 1120 & $1,193(25)$ & $1,185(23)$ & $1,220(13)$ \\
\hline \multirow{12}{*}{2010} & \multirow{2}{*}{${ }^{95} \mathrm{Zr}$} & 724,2 & $8,88(12) \times 10^{-05}$ & $8,89(9) \times 10^{-05}$ & $8,99(10) \times 10^{-5}$ \\
\hline & & 756,7 & $1,10(1) \times 10^{-04}$ & $1,10(1) \times 10^{-04}$ & $1,11(2) \times 10^{-4}$ \\
\hline & ${ }^{65} \mathrm{Zn}$ & 1115,5 & $5,60(9) \times 10^{-03}$ & $5,58(5) \times 10^{-03}$ & $5,72(2) \times 10^{-3}$ \\
\hline & $69 \mathrm{~m} \mathrm{Zn}$ & 438,6 & $3,94(10) \times 10^{-4}$ & $3,93(9) \times 10^{-04}$ & $3,98(2) \times 10^{-4}$ \\
\hline & \multirow{4}{*}{${ }^{140} \mathrm{La}$} & 328,7 & $2,87(4) \times 10^{-02}$ & $2,89(3) \times 10^{-02}$ & $2,87(3) \times 10^{-2}$ \\
\hline & & 487 & $6,40(9) \times 10^{-02}$ & $6,42(6) \times 10^{-02}$ & $6,37(6) \times 10^{-2}$ \\
\hline & & 815,8 & $3,35(5) \times 10^{-02}$ & $3,33(3) \times 10^{-02}$ & $3,32(2) \times 10^{-2}$ \\
\hline & & 1596,2 & $1,34(2) \times 10^{-01}$ & $1,38(3) \times 10^{-01}$ & $1,34(1) \times 10^{-1}$ \\
\hline & \multirow{2}{*}{${ }^{60} \mathrm{Co}$} & 1173,2 & $1,330(21)$ & $1,344(16)$ & $1,320(5)$ \\
\hline & & 1332,5 & $1,322(22)$ & $1,320(17)$ & $1,320(7)$ \\
\hline & \multirow{2}{*}{${ }^{46} \mathrm{Sc}$} & 889 & $1,218(17)$ & $1,237(13)$ & $1,220(5)$ \\
\hline & & 1120 & $1,224(17)$ & $1,225(12)$ & $1,220(13)$ \\
\hline
\end{tabular}

Observa-se que ambos os códigos utilizados exibiram valores aproximados, indicando que não houve divergências nos dados ou nos próprios códigos propostos no presente trabalho. Verificou-se também, pelas TAB. apresentadas, que o código AKFIT v2.1 obteve incertezas menores, o que pode sugerir um potencial de ser mais preciso.

Definiu-se, no presente trabalho, as médias de todos os métodos utilizados como os valores gerais de $k_{0}$ para cada elemento. Ou seja, realizou-se a média dos valores de $k_{0}$ por meio dos resultados obtidos pelos campos $\mathrm{K} 0 \mathrm{e}$ K03 do código COVAR v4.1 (FIG. 6) e dos resultados obtidos pelo AKFIT v2.1 por meio dos ajustes individuais e simultâneo dos dados analisados. Para os isótopos que estavam contidos em ambas as irradiações, realizou-se uma nova média entre os valores obtidos em cada período. O resultado é apresentado na TAB. 21: 
TABELA 21 - Valores médios de $k_{0}$ calculados para os elementos analisados.

\begin{tabular}{|c|c|c|c|}
\hline \multicolumn{4}{|c|}{ Valores de $\boldsymbol{k}_{0}$} \\
\hline Produto & Energia & $k_{0}$ (Presente Trabalho) & 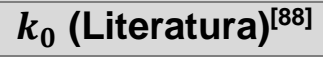 \\
\hline \multirow{2}{*}{${ }^{95} \mathrm{Zr}$} & 724,2 & $8,92(12) \times 10^{-05}$ & $8,99(10) \times 10^{-5}$ \\
\hline & 756,7 & $1,11(2) \times 10^{-04}$ & $1,11(2) \times 10^{-4}$ \\
\hline${ }^{65} \mathrm{Zn}$ & 1115,5 & $5,63(8) \times 10^{-03}$ & $5,72(2) \times 10^{-3}$ \\
\hline $69 \mathrm{~m} \mathrm{Zn}$ & 438,6 & $3,93(9) \times 10^{-04}$ & $3,98(2) \times 10^{-4}$ \\
\hline \multirow{4}{*}{${ }^{140} \mathrm{La}$} & 328,7 & $2,86(4) \times 10^{-02}$ & $2,87(3) \times 10^{-2}$ \\
\hline & 487 & $6,39(8) \times 10^{-02}$ & $6,37(6) \times 10^{-2}$ \\
\hline & 815,8 & $3,33(4) \times 10^{-02}$ & $3,32(2) \times 10^{-2}$ \\
\hline & 1596,2 & $1,36(3) \times 10^{-01}$ & $1,34(1) \times 10^{-1}$ \\
\hline \multirow{2}{*}{${ }^{60} \mathrm{Co}$} & 1173,2 & $1,334(18)$ & $1,320(5)$ \\
\hline & 1332,5 & $1,321(19)$ & $1,320(7)$ \\
\hline \multirow{2}{*}{${ }^{46} \mathrm{Sc}$} & 889 & $1,209(19)$ & $1,220(5)$ \\
\hline & 1120 & $1,208(18)$ & $1,220(13)$ \\
\hline
\end{tabular}

Para o parâmetro $\alpha$ o valor médio obtido foi $0,0025 \pm 0,0083$. Pode-se evidenciar que todos os parâmetros calculados estão de acordo com os valores referenciais usados neste trabalho.

À nível de comparação, na TAB. 22 é apresentado os valores de ${ }^{65} \mathrm{Zn}$ da referência ${ }^{[17,18]}$ e os obtidos no presente trabalho:

TABELA 22 - Comparação dos valores de $k_{0}$ e $Q_{0}$ para ${ }^{64} \mathrm{Zn}$ da literatura com os obtidos no presente trabalho.

\begin{tabular}{ccccc}
\hline & \multicolumn{4}{c}{ Comparação de ${ }^{64} \mathbf{Z n}$} \\
Alvo & Parâm. & Ref. $^{[17,18]}$. & Ref. $^{[88]}$ & Presente Trabalho \\
\hline \multirow{2}{*}{${ }^{64} \mathrm{Zn}$} & $k_{0}$ & $5,63(8) \times 10^{-3}$ & $5,72(2) \times 10^{-3}$ & $5,63(8) \times 10^{-3}$ \\
\cline { 2 - 5 } & $Q_{0}$ & $1,69(6)$ & $1,91(9)$ & $1,88(5)$ \\
\hline
\end{tabular}

O valor de $k_{0}$ obtido para a irradiação de 2008 foi exatamente o mesmo em relação à referência ${ }^{[18]}$, entretanto, o valor de $Q_{0}$ foi maior, se aproximando da referência IUPAC utilizada. 
O objetivo dos resultados apresentados neste trabalho procurou verificar a validade da construção do novo código incluído no COVAR v4.1 e do código AKFIT v2.1. Com atualização para COVAR v4.1, corrigindo-se algumas limitações da versão anterior (COVAR v3), como a limitação da análise de, no máximo, 20 alvos e a limitação das análises das correlações criando uma extensão para qualquer caso geral de arquivos de dados, criou-se um código mais completo e eficiente. Os valores obtidos pela nova equação incluída neste trabalho foram semelhantes aos resultados da equação existente na versão anterior, indicando que os cálculos estão em acordo com os resultados validados pelo código, sendo possível usá-lo como uma forma alternativa de análise, já que não se faz necessário a utilização das irradiações com cádmio, na condição de que se saibam os valores de $f$ e $Q_{0, i}$ previamente.

A criação dos métodos linear e não linear de ajuste para o código AKFIT v2.1 possibilitou a determinação dos parâmetros $\alpha$ e $k_{0}$ de modo único e eficiente. Ambos os ajustes se mostraram coerentes com o previsto, obtendo resultados satisfatórios e com incertezas ligeiramente menores que o código COVAR v4.1. É interessante realizar novos testes.

As matrizes de correlação geradas neste trabalho foram importantes para se averiguar o comportamento dos dados, auxiliando na verificação de eventuais erros de análise ou de medida. Por meio delas foi possível verificar as correlações de cada parâmetro envolvido na AAN que foi utilizado.

Com base no trabalho realizado concluiu-se que não houve erros nos códigos desenvolvidos e todos os resultados estão dentro do esperado logicamente, conforme as informações impostas no arquivo de dados de entrada. Este estudo, além de aplicar toda a metodologia de análise por covariância, contribuiu para a melhoria dos dados nucleares para este tipo de aplicação. Os sucessos dos códigos mostraram a possibilidade dos seus usos de modo eficiente e confiável. 
Em trabalhos futuros, sugere-se incluir mais parâmetros para as correlações e assim obter uma análise mais completa e detalhada dos dados para ambos os códigos. Sugere-se ainda para o AKFIT v2.1 a inclusão dos parâmetros $Q_{0, i}$ e $f$ em seu código, pois, já possuindo um ajuste não-linear, torna-se viável suas inclusões, a fim de criar um código capaz de calcular os parâmetros essenciais do Método $k_{0}$. Pretende-se também que tanto COVAR v4 quanto os ajustes únicos sejam incorporados em um só código, ou seja, criar um programa único que possua diferentes formas de obtenção do fator $k_{0}$ e os parâmetros associados, tornando-o um programa de uso padrão, manipulável e completo, podendo ser usado como referência para as determinações dos parâmetros necessários da AAN, sem a necessidade de recorrer a outros métodos de cálculos externos, como uso de planilhas e objetos afins. 


\section{APÊNDICES}

\section{Apêndice A}

Listagem parcial do código COVAR v4.1, apresentando as atualizações realizadas na determinação das covariâncias entre os parâmetros $k_{0}$ e $Q_{0}$ e a inclusão da EQ. (3.3).

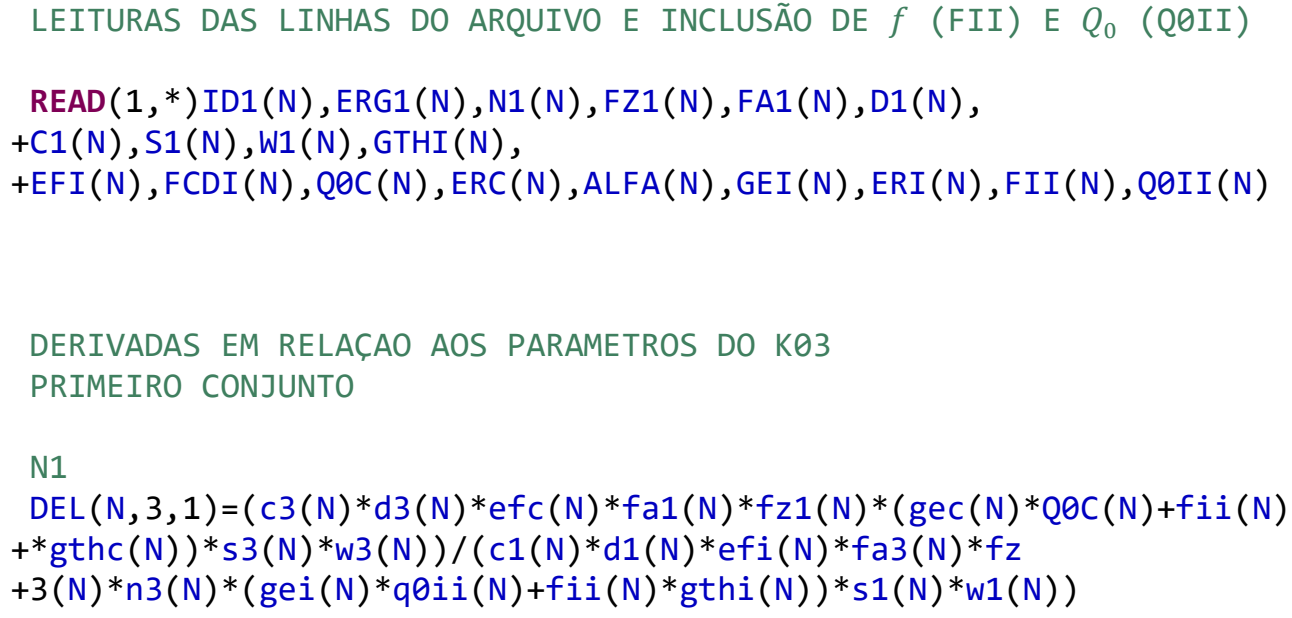




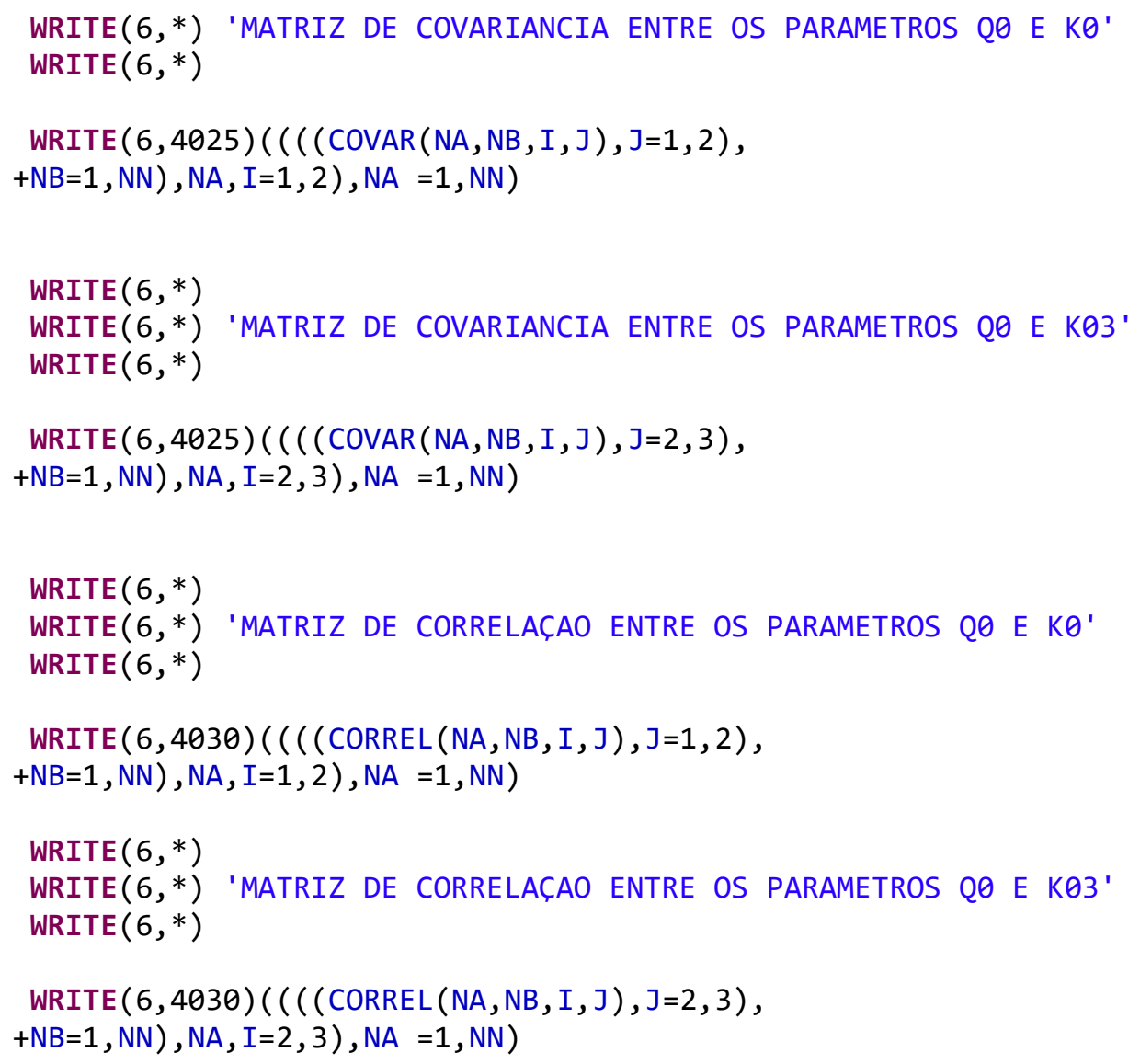




\section{Apêndice B}

\section{Apresentação do código AKFIT v2.1, baseado no código modelo criado por MORALLES por meio do programa SCILAB.}

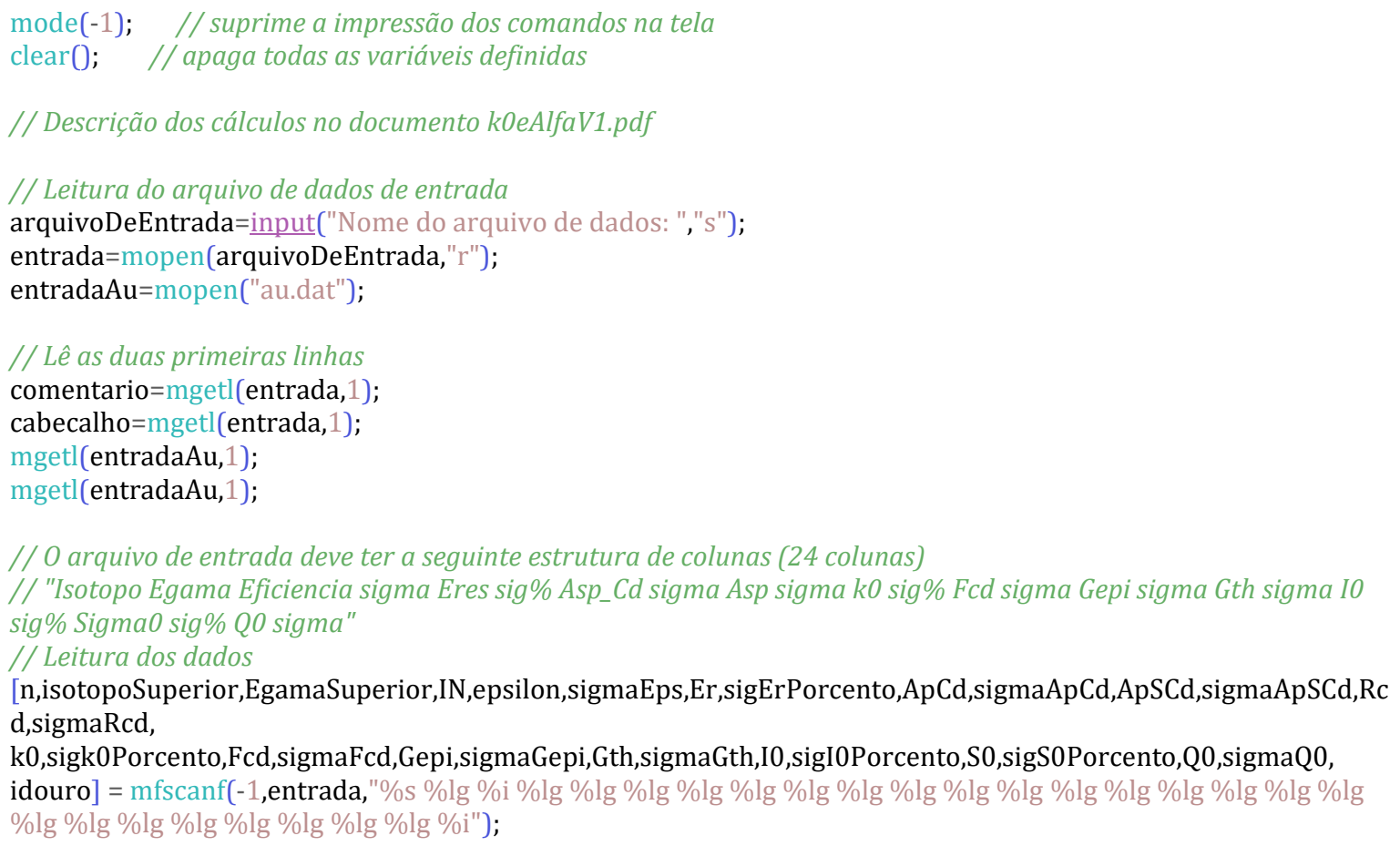

[nAu,isotopoSuperiorAu,EgamaSuperiorAu,INAu,epsilonAu,sigmaEpsAu,ErAu,sigErPorcentoAu,ApCdAu,sigmaApC $\mathrm{dAu}, \mathrm{ApSCdAu,sigmaApSCdAu,RcdAu,sigmaRcdAu,}$

k0Au,sigk0PorcentoAu,FcdAu,sigmaFcdAu,GepiAu,sigmaGepiAu,GthAu,sigmaGthAu,IOAu,sigI0PorcentoAu,S0Au,si gS0PorcentoAu,Q0Au,sigmaQ0Au] = mfscanf(-1,entradaAu,"\%s \%lg \%i \%lg \%lg \%lg \%lg \%lg \%lg \%lg \%lg \%lg $\% \lg \% \lg \% \lg \% \lg \% \lg \% \lg \% \lg \% \lg \% \lg \% \lg \% \lg \% \lg \% \lg \% \lg \% \lg$ );

// ---- Cálculo de alfa e dos k0 simultaneamente

// Usando a aproximação linear de QO

for $\mathbf{i}=1$ : NGamas

isotopoInferior $(\mathrm{i})=$ isotopoSuperior $(\mathrm{i})$;

EgamaInferior (i) = EgamaSuperior (i); end;

for $\mathrm{i}=1$ : NGamas

Ysuperior(i)= $\log (\operatorname{ApCd}(\mathrm{i}) . / \mathrm{Q} 0(\mathrm{i}) . /$ epsilon(i)./Fcd(i)./Gepi(i)); end;

//Ysuperior()=log(ApCd./Q0./epsilon./Fcd./Gepi);

//

for $\mathbf{i}=1$ :NGamas

Yinferior(i) $=\log ((\operatorname{ApSCd}(\mathrm{i})-\operatorname{ApCd}(\mathrm{i}) \cdot / \mathrm{Fcd}(\mathrm{i})) \cdot /(\operatorname{ApSCdAu}($ idouro(i))-

ApCdAu(idouro(i))./FcdAu(idouro(i))).*GthAu(idouro(i))./Gth(i).*epsilonAu(idouro(i))./epsilon(i)); end;

// Cálculos das derivadas parciais

// Ysuperior

dYsdApCd = ApCd.^(-1);

$\mathrm{dYsdQ0}=-\mathrm{Q} 0{ }^{\wedge}(-1)$; 


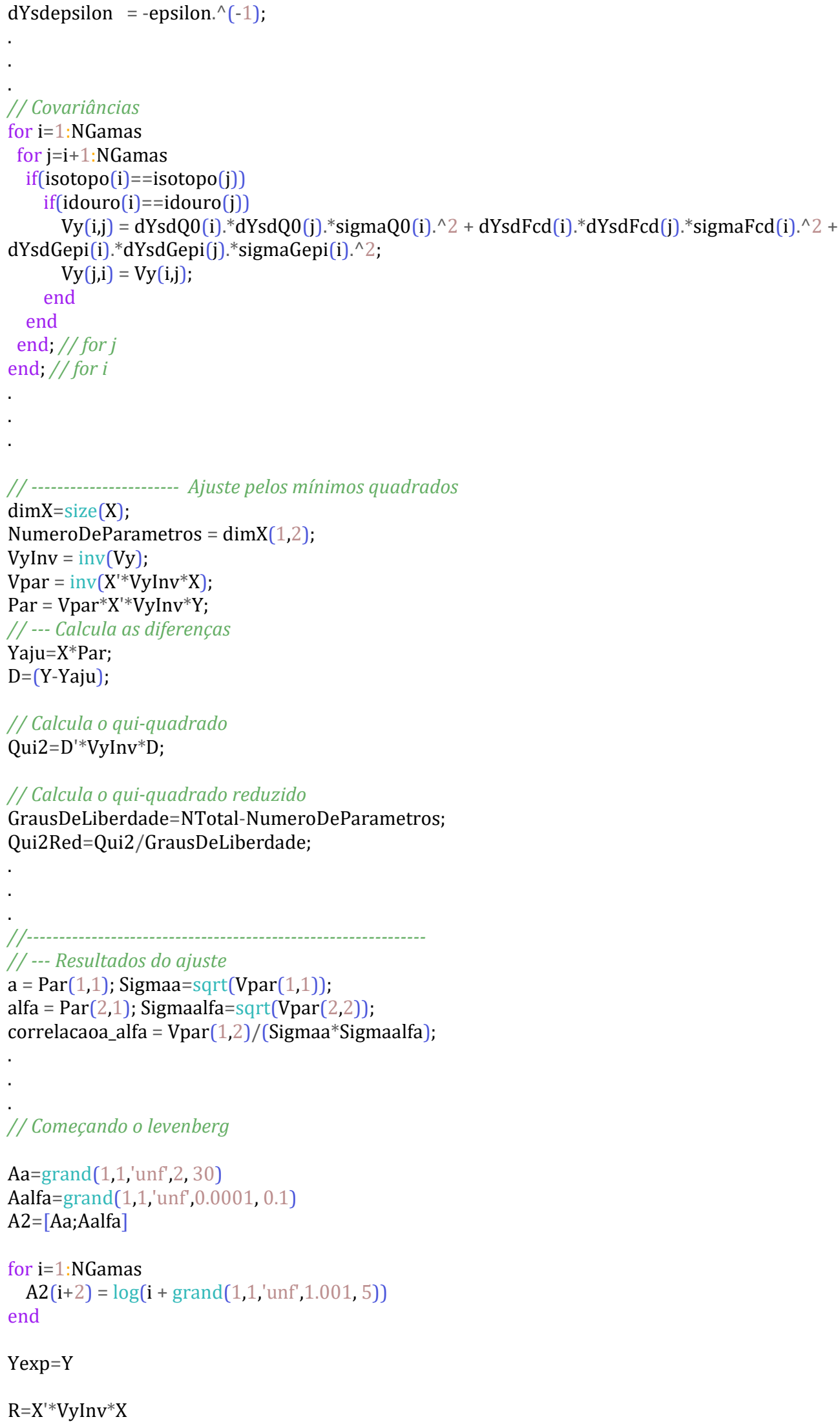




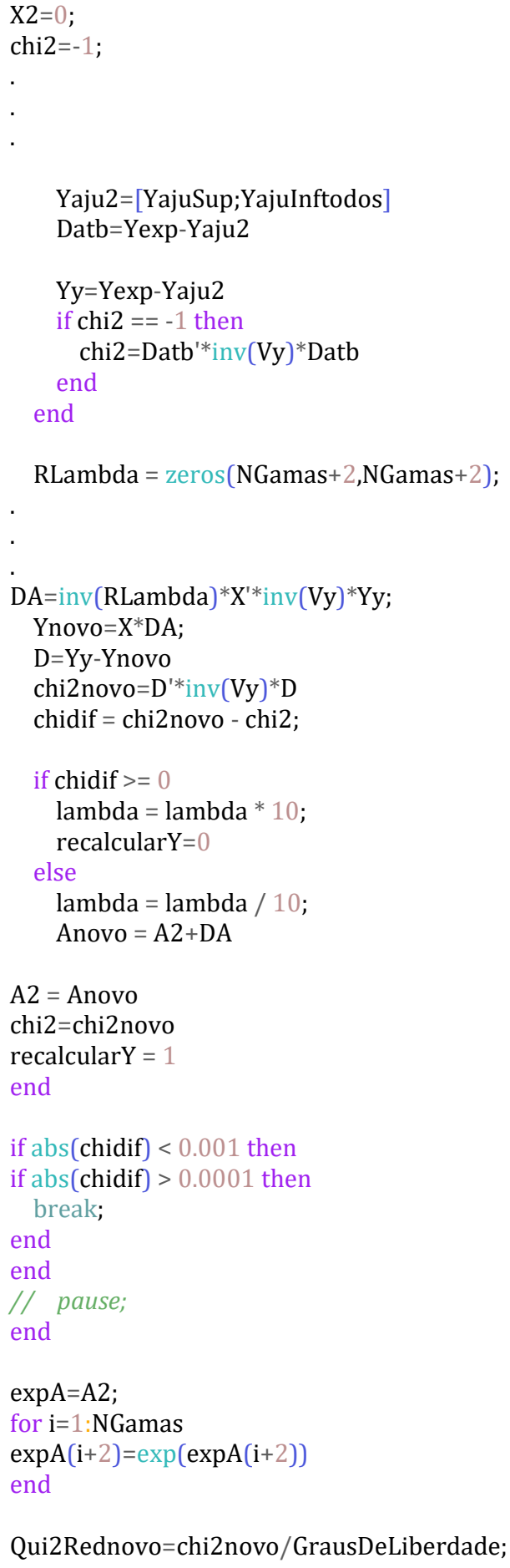




\section{Apêndices C, D, E e F}

As matrizes apresentadas a seguir, por serem muito extensas, foram divididas em partes para que seja possível observá-las. A divisão foi realizada de acordo com o esquema apresentado na FIG. 13.

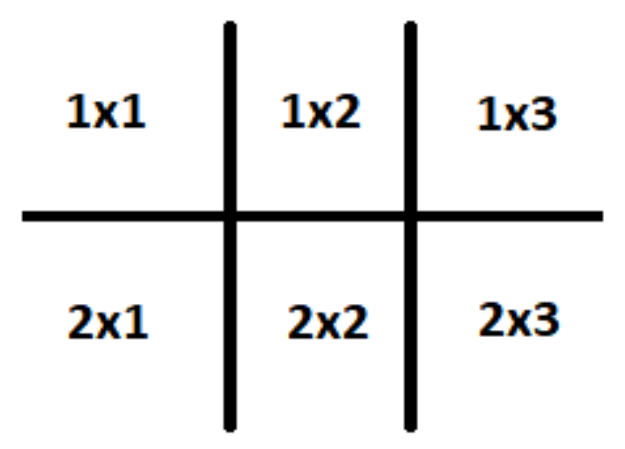

FIGURA 13 - Esquema da divisão realizada nas matrizes com suas partes definidas.

Na situação em que a matriz é menor, não foi necessário fazer muitas divisões, bastando em dividi-la na quantidade que for conveniente.

No Apêndice C é apresentado a matriz de correlações do código COVAR v4.1 para os parâmetros $k_{0}(\mathrm{~K} 0)$ e $Q_{0}$. No Apêndice $\mathrm{D}$, apresenta-se a Matriz para a relação entre os parâmetros $k_{0}(\mathrm{~K} 03)$ e $Q_{0}$. No Apêndice $\mathrm{E}$ é apresentado a matriz de correlação entre os parâmetros $a, \alpha$ e $k_{0}$ de todos os elementos dos anos de 2008 e 2010 analisados simultaneamente. E no apêndice F estão apresentadas as Matrizes dos dados de 2008 e 2010 analisados separadamente. 


\section{Apêndice C}

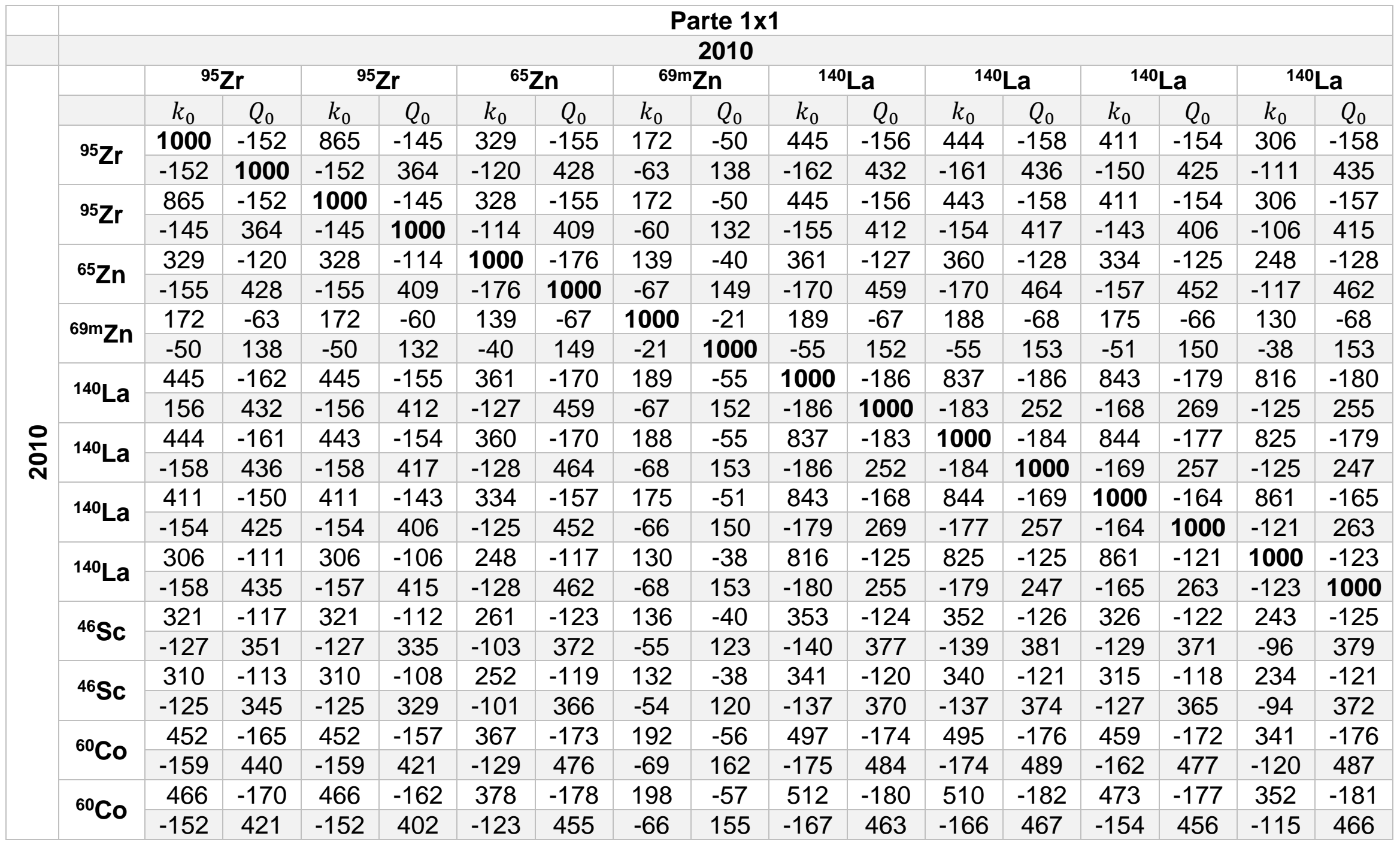




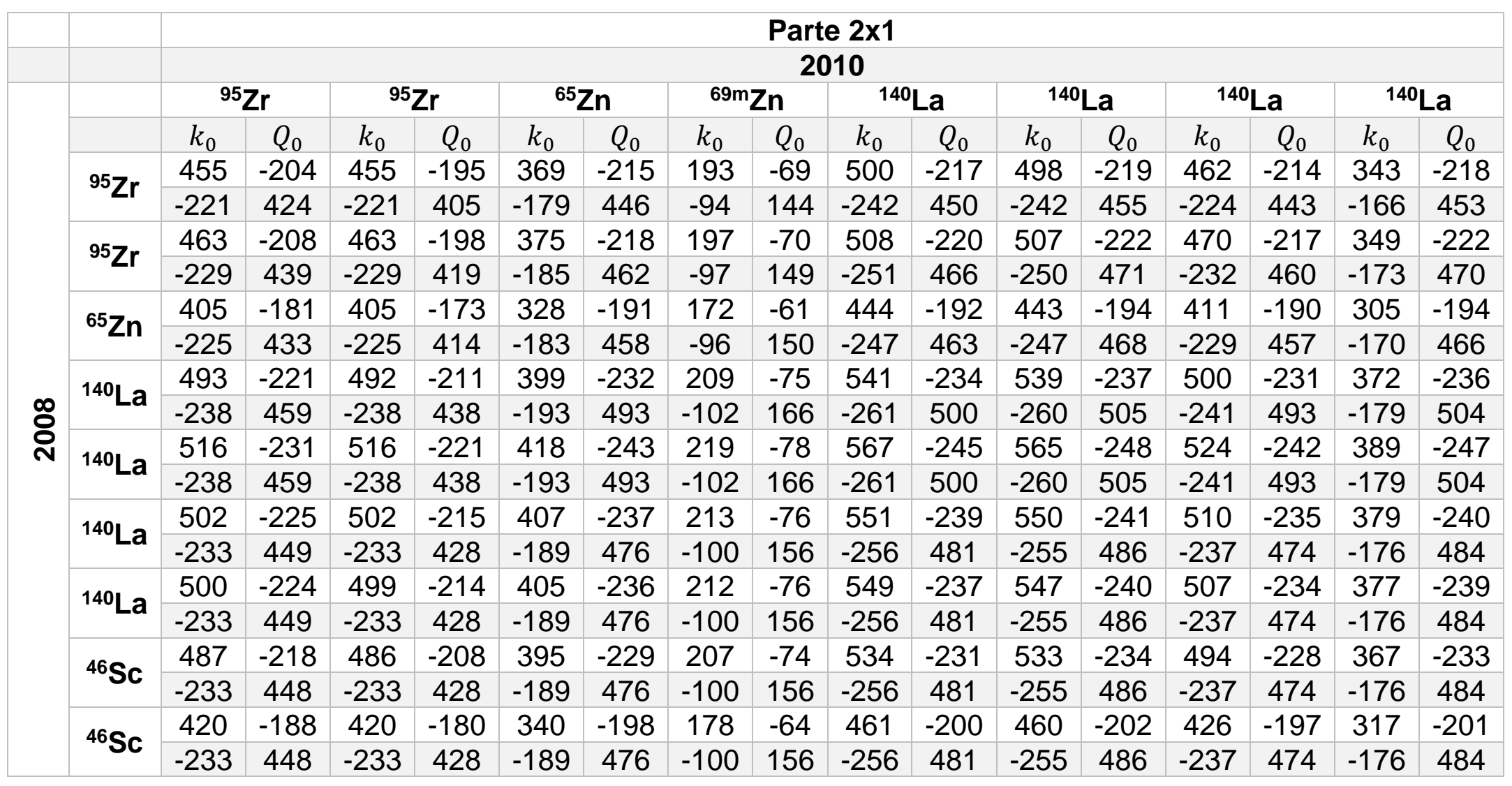




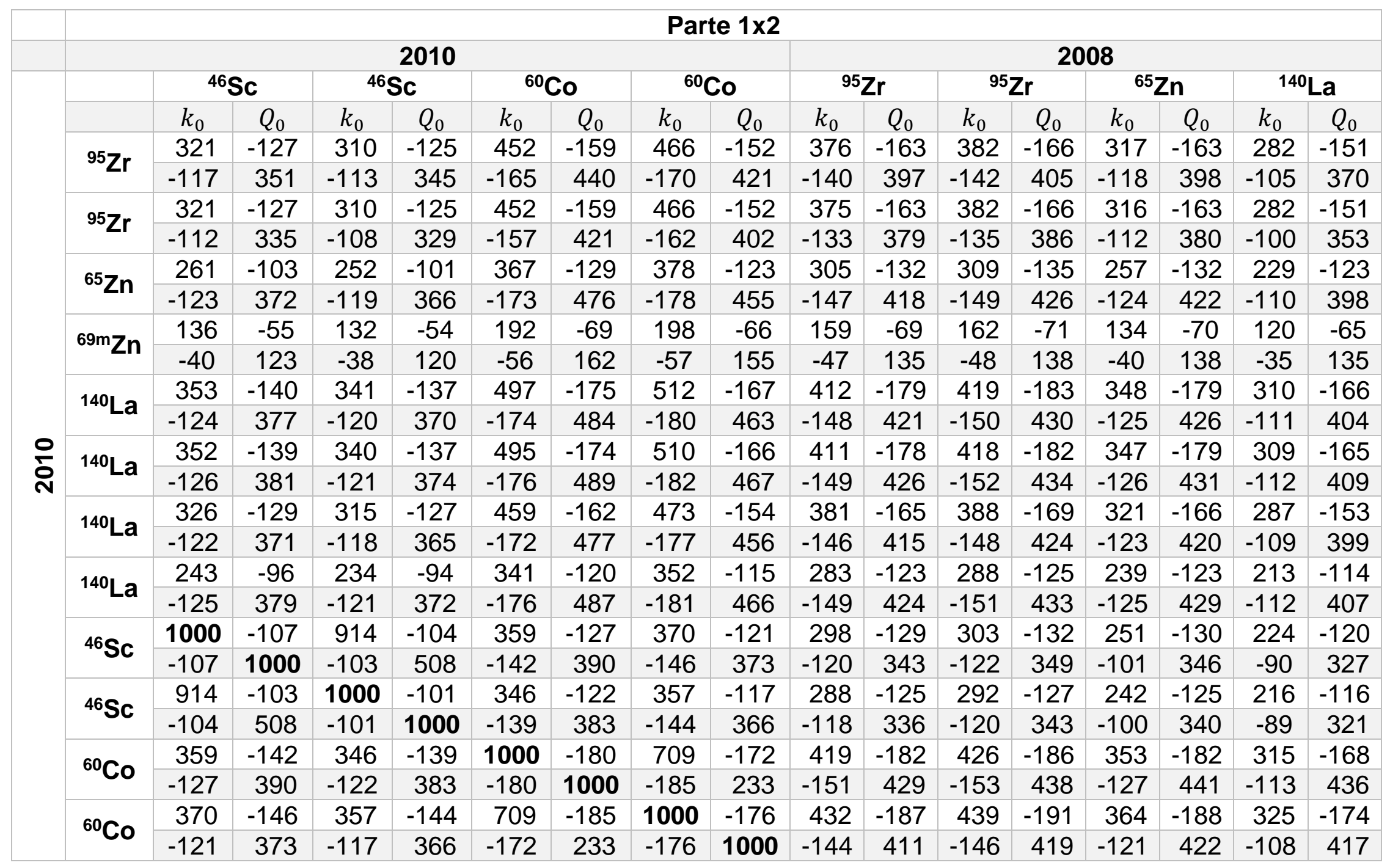




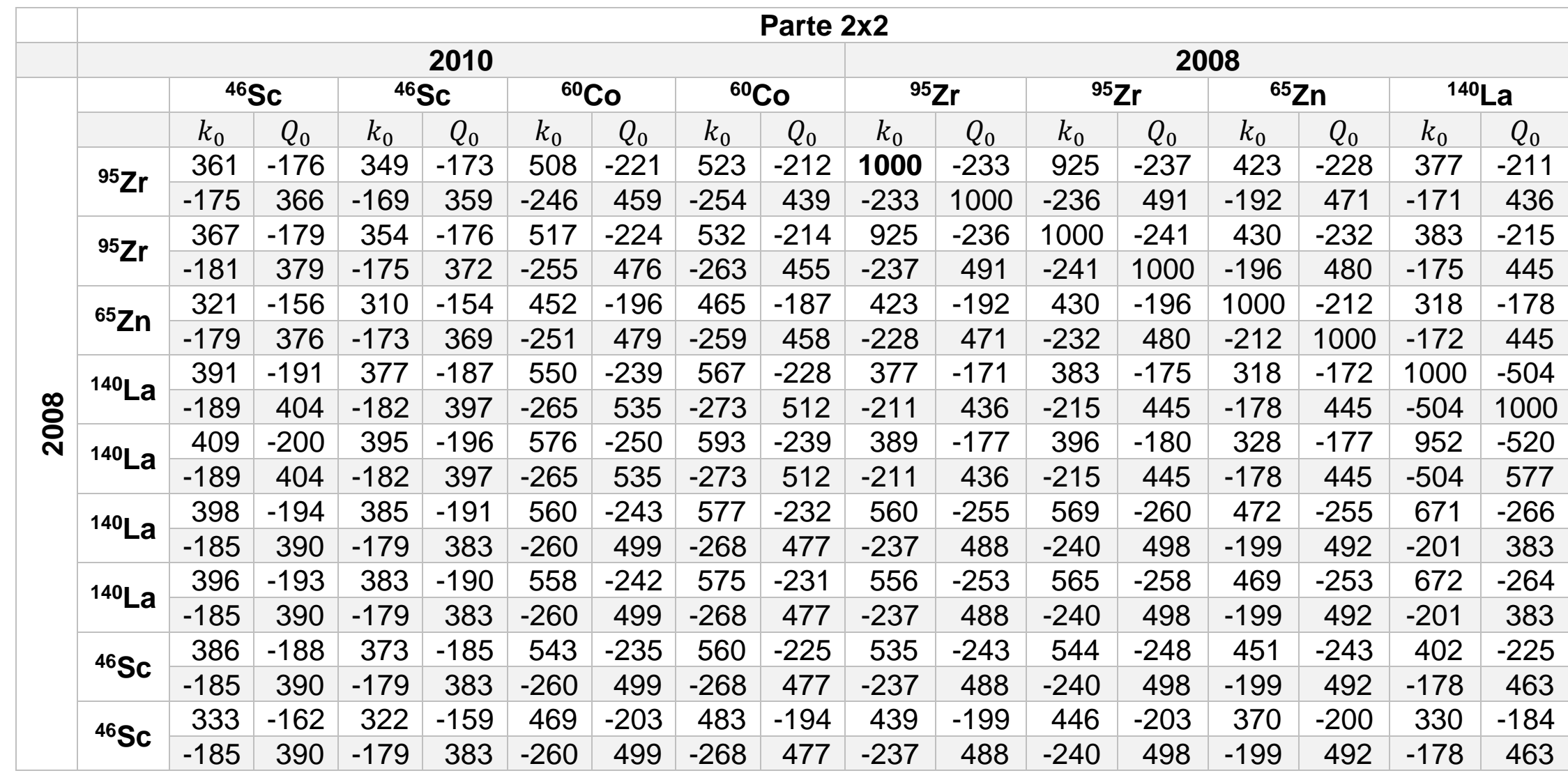




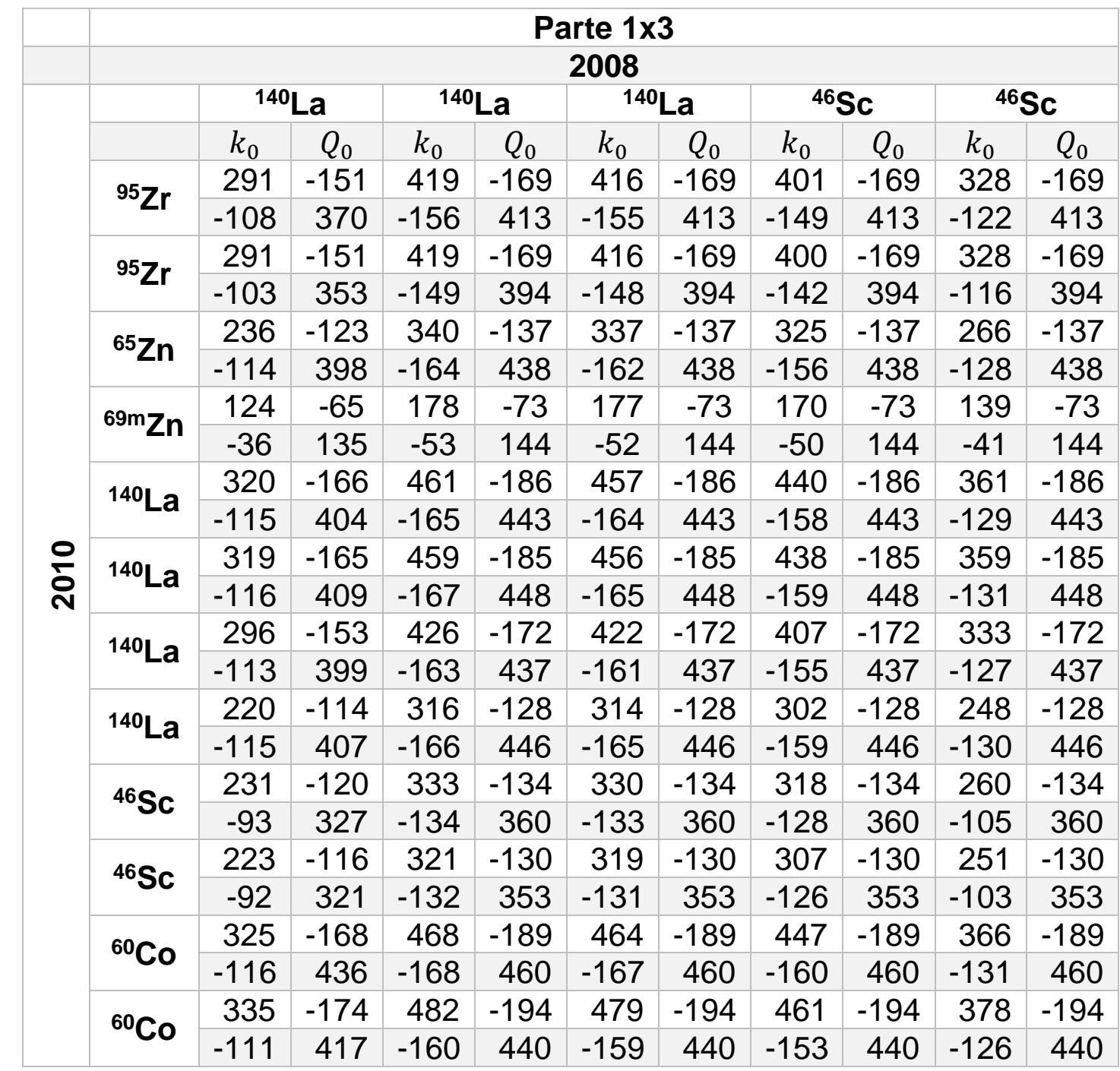




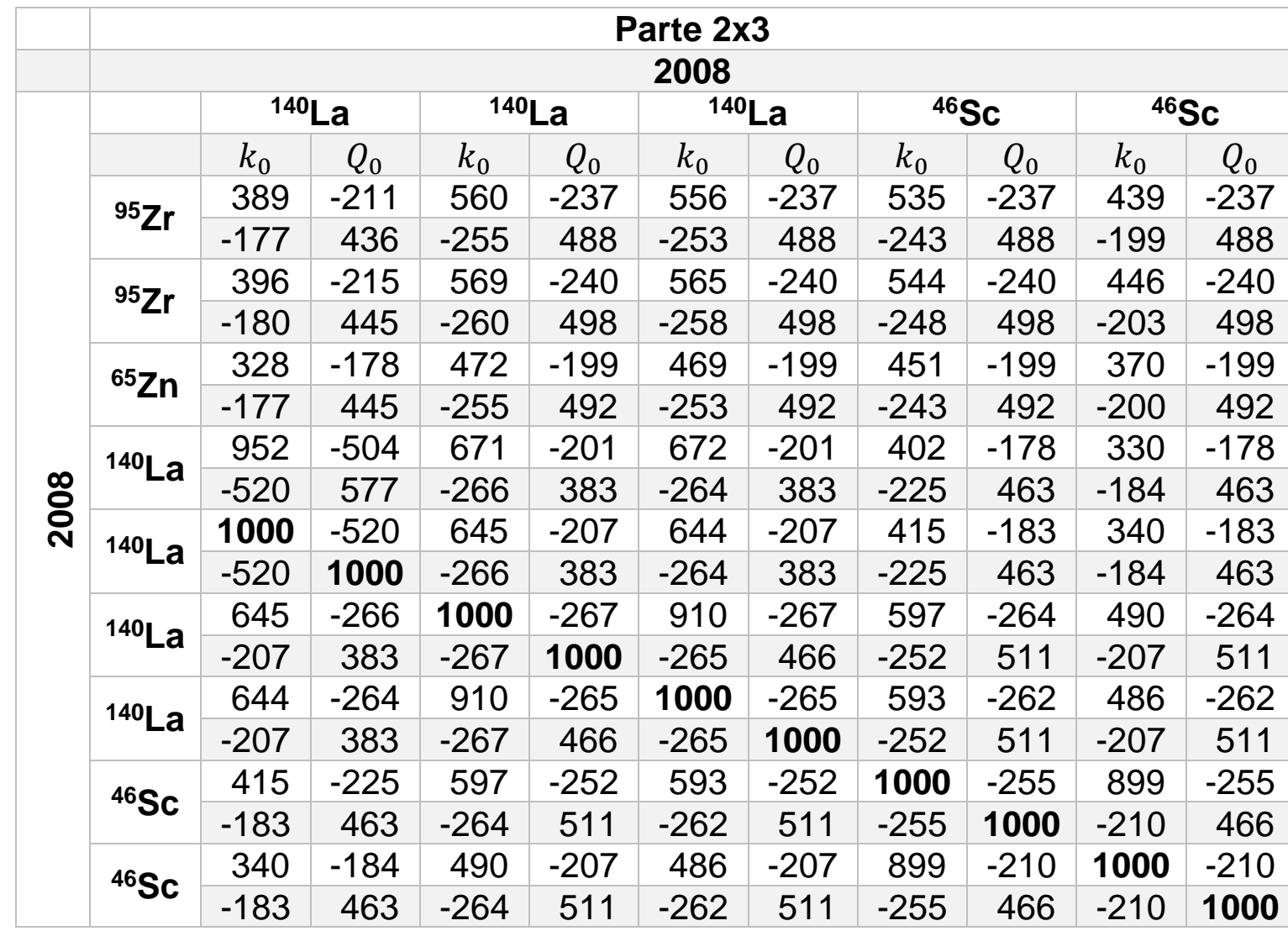




\section{Apêndice D}

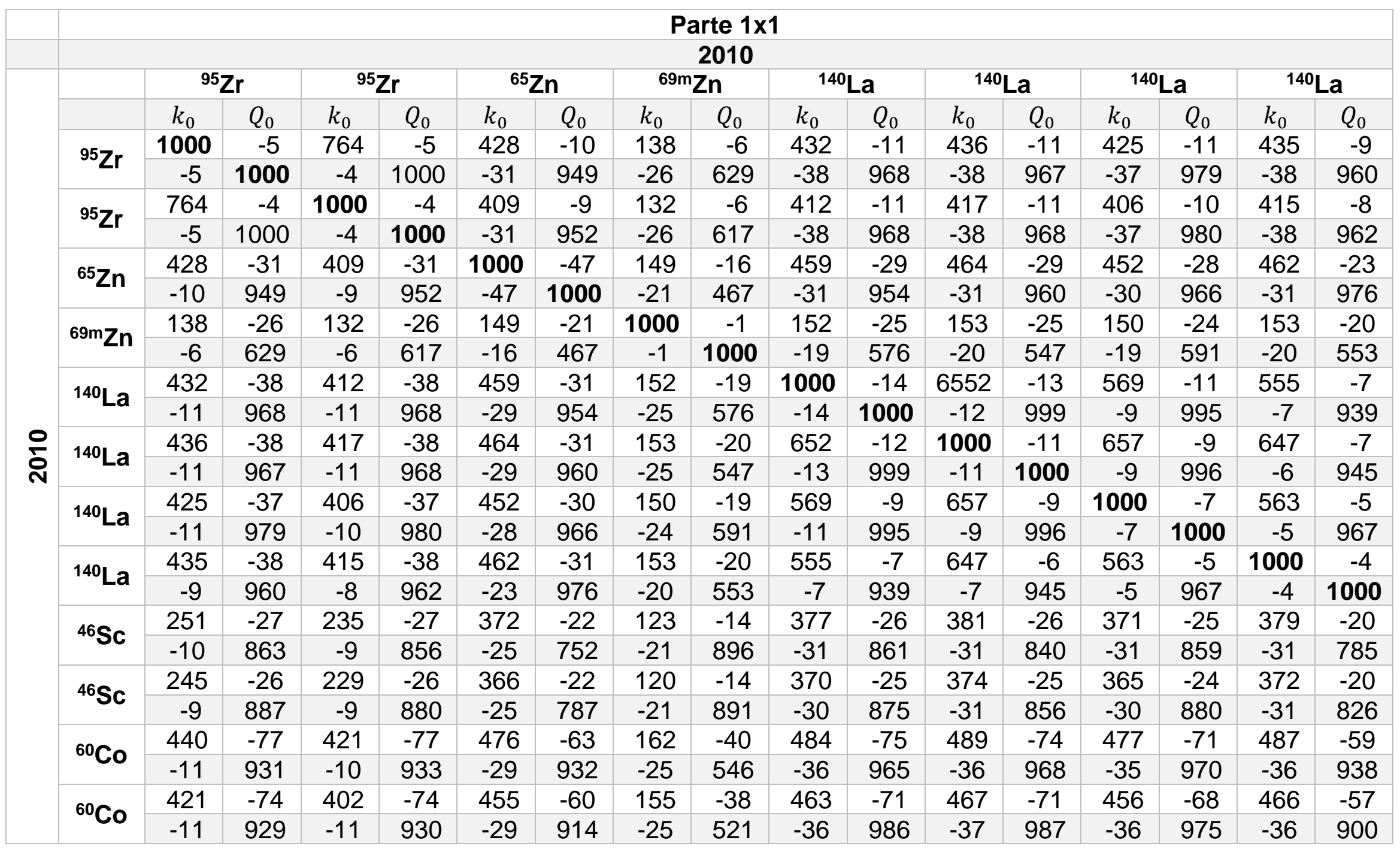




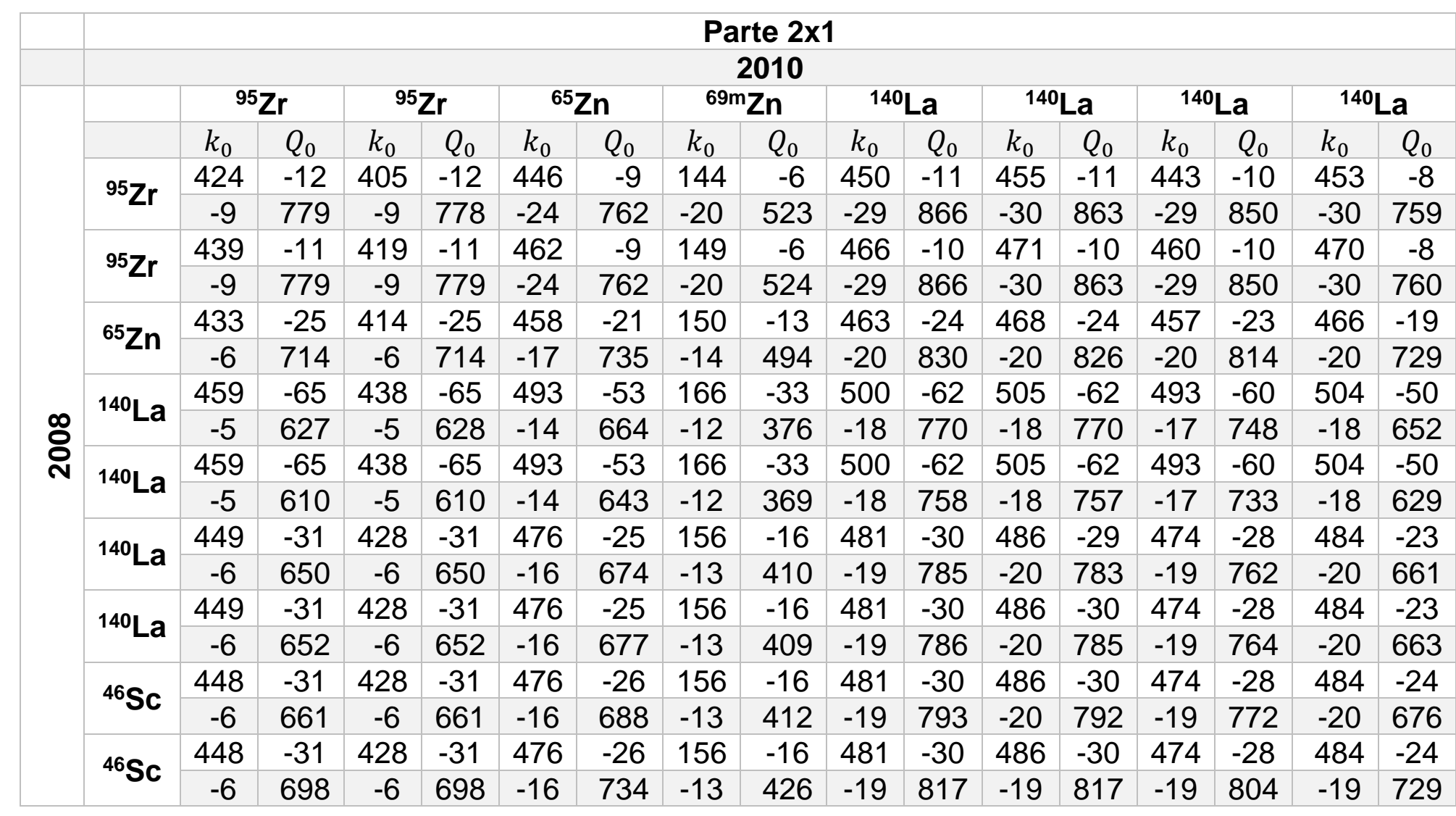




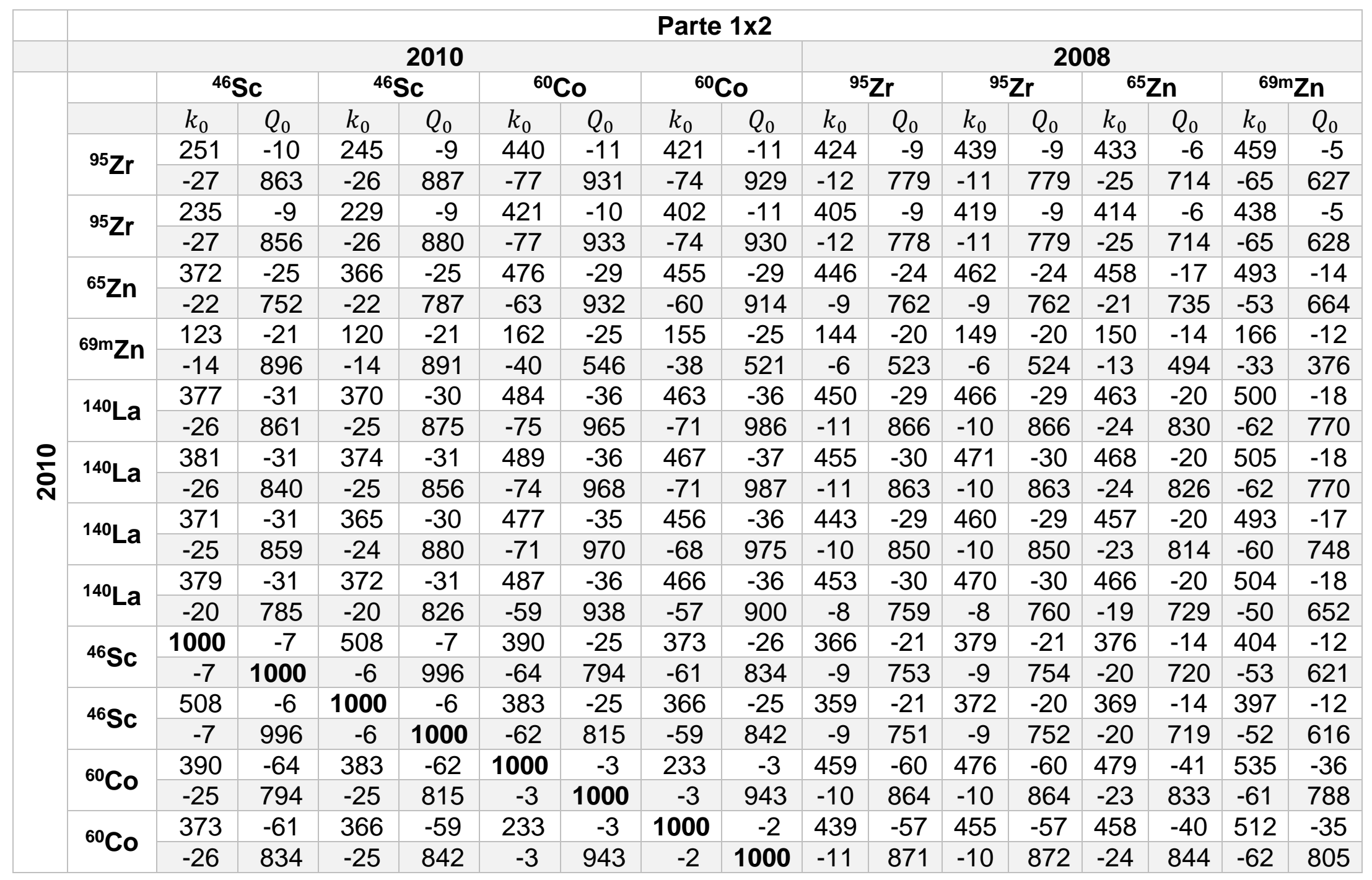




\begin{tabular}{|c|c|c|c|c|c|c|c|c|c|c|c|c|c|c|c|c|c|}
\hline & \multicolumn{17}{|c|}{ Parte 2x2 } \\
\hline & \multicolumn{9}{|c|}{2010} & \multicolumn{8}{|c|}{2008} \\
\hline \multirow{20}{*}{$\begin{array}{l}\infty \\
\text { O্ণ } \\
\text { N }\end{array}$} & & \multicolumn{2}{|c|}{${ }^{46} \mathrm{Sc}$} & \multicolumn{2}{|c|}{${ }^{46} \mathrm{Sc}$} & \multicolumn{2}{|c|}{${ }^{60} \mathrm{Co}$} & \multicolumn{2}{|c|}{${ }^{60} \mathrm{Co}$} & \multicolumn{2}{|c|}{${ }^{95} \mathrm{Zr}$} & \multicolumn{2}{|c|}{${ }^{95} \mathrm{Zr}$} & \multicolumn{2}{|c|}{$65 \mathrm{Zn}$} & \multicolumn{2}{|c|}{${ }^{140} \mathrm{La}$} \\
\hline & & $k_{0}$ & $Q_{0}$ & $k_{0}$ & $Q_{0}$ & $k_{0}$ & $Q_{0}$ & $k_{0}$ & $Q_{0}$ & $k_{0}$ & $Q_{0}$ & $k_{0}$ & $Q_{0}$ & $k_{0}$ & $Q_{0}$ & $k_{0}$ & $Q_{0}$ \\
\hline & \multirow{2}{*}{${ }^{95} \mathbf{Z r}$} & 366 & -9 & 359 & -9 & 459 & -10 & 439 & -11 & 1000 & 6 & 491 & 7 & 471 & 119 & 436 & 91 \\
\hline & & -21 & 753 & -21 & 751 & -60 & 864 & -57 & 871 & 6 & 1000 & 6 & 999 & 87 & 973 & 50 & 742 \\
\hline & \multirow{2}{*}{${ }^{95} \mathrm{Zr}$} & 379 & -9 & 372 & -9 & 476 & -10 & 455 & -10 & 491 & 6 & 1000 & 7 & 480 & 122 & 445 & 93 \\
\hline & & -21 & 754 & -20 & 752 & -60 & 864 & -57 & 872 & 7 & 999 & 7 & 1000 & 88 & 973 & 51 & 750 \\
\hline & \multirow{2}{*}{${ }^{65} \mathrm{Zn}$} & 376 & -20 & 369 & -20 & 479 & -23 & 458 & -24 & 471 & 87 & 480 & 88 & 1000 & 7 & 445 & 81 \\
\hline & & -14 & 720 & -14 & 719 & -41 & 833 & -40 & 844 & 119 & 973 & 122 & 973 & 7 & 1000 & 75 & 738 \\
\hline & \multirow{2}{*}{${ }^{140} \mathrm{La}$} & 404 & -53 & 397 & -52 & 535 & -61 & 512 & -62 & 436 & 50 & 445 & 51 & 445 & 75 & 1000 & -309 \\
\hline & & -12 & 621 & -12 & 616 & -36 & 788 & -35 & 805 & 91 & 742 & 93 & 750 & 81 & 738 & -309 & 1000 \\
\hline & \multirow{2}{*}{${ }^{140} \mathrm{La}$} & 404 & -53 & 397 & -52 & 535 & -61 & 512 & -62 & 436 & 50 & 445 & 51 & 445 & 75 & 577 & -309 \\
\hline & & -13 & 613 & -12 & 605 & -36 & 773 & -35 & 796 & 79 & 717 & 81 & 724 & 69 & 703 & -330 & 995 \\
\hline & \multirow{2}{*}{${ }^{140} \mathrm{La}$} & 390 & -25 & 383 & -25 & 499 & -29 & 477 & -29 & 488 & 85 & 498 & 86 & 492 & 108 & 383 & -19 \\
\hline & & -14 & 649 & -14 & 642 & -40 & 797 & -38 & 813 & 110 & 957 & 112 & 962 & 97 & 950 & -31 & 790 \\
\hline & \multirow{2}{*}{${ }^{140} \mathrm{La}$} & 390 & -25 & 383 & -25 & 499 & -29 & 477 & -30 & 488 & 85 & 498 & 86 & 492 & 108 & 383 & -19 \\
\hline & & -14 & 649 & -14 & 643 & -40 & 799 & -38 & 814 & 111 & 958 & 114 & 964 & 98 & 953 & -29 & 790 \\
\hline & \multirow{2}{*}{${ }^{46} \mathrm{Sc}$} & 390 & -26 & 383 & -25 & 499 & -29 & 477 & -30 & 488 & 85 & 498 & 86 & 492 & 108 & 463 & 80 \\
\hline & & -14 & 652 & -14 & 648 & -40 & 806 & -38 & 819 & 118 & 962 & 120 & 969 & 105 & 963 & 68 & 790 \\
\hline & \multirow{2}{*}{${ }^{46} \mathrm{Sc}$} & 390 & -26 & 383 & -25 & 499 & -29 & 477 & -30 & 488 & 85 & 498 & 86 & 492 & 108 & 463 & 80 \\
\hline & & -14 & 665 & -13 & 668 & -39 & 836 & -38 & 835 & 137 & 943 & 140 & 951 & 126 & 966 & 91 & 759 \\
\hline
\end{tabular}




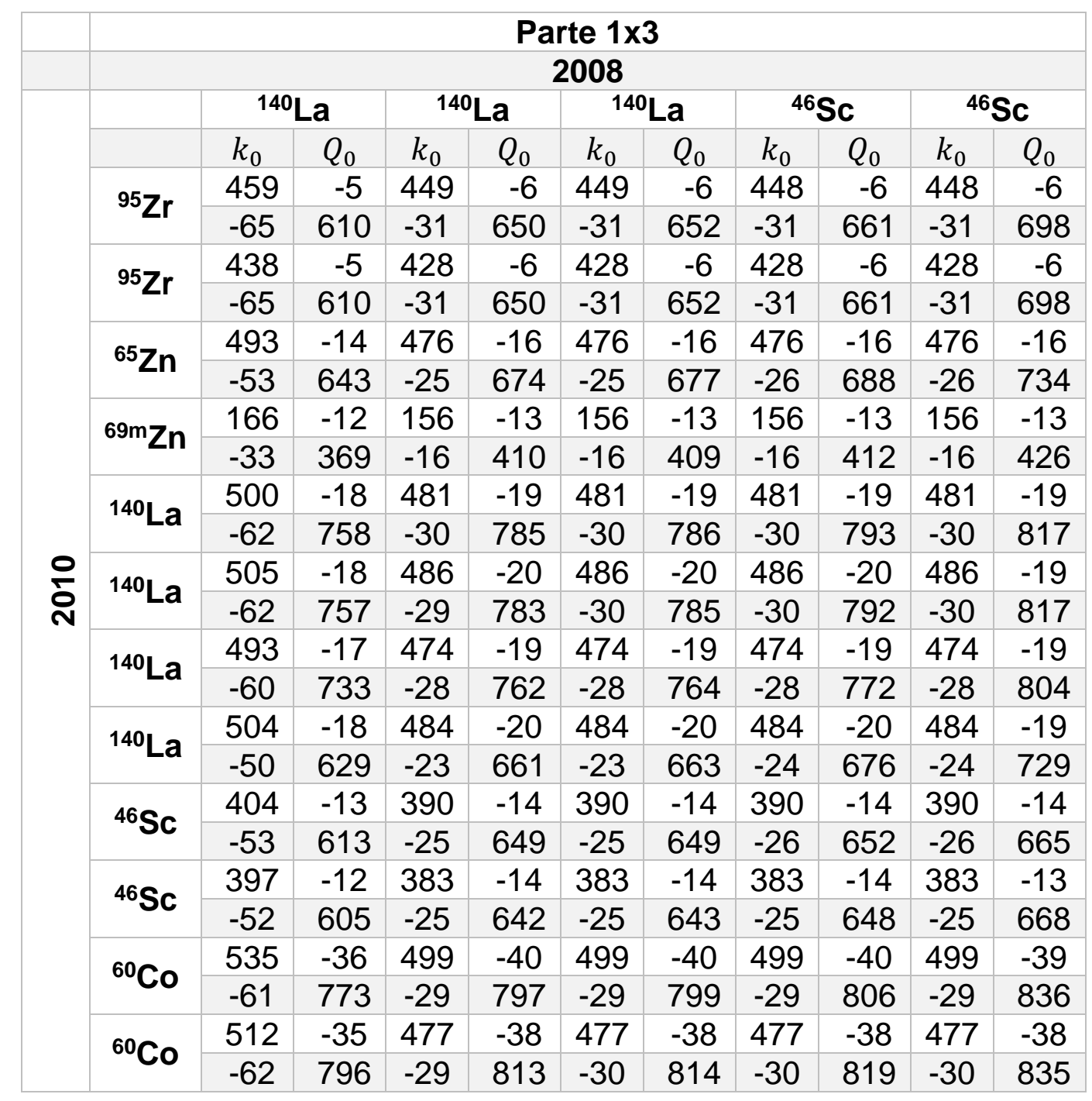




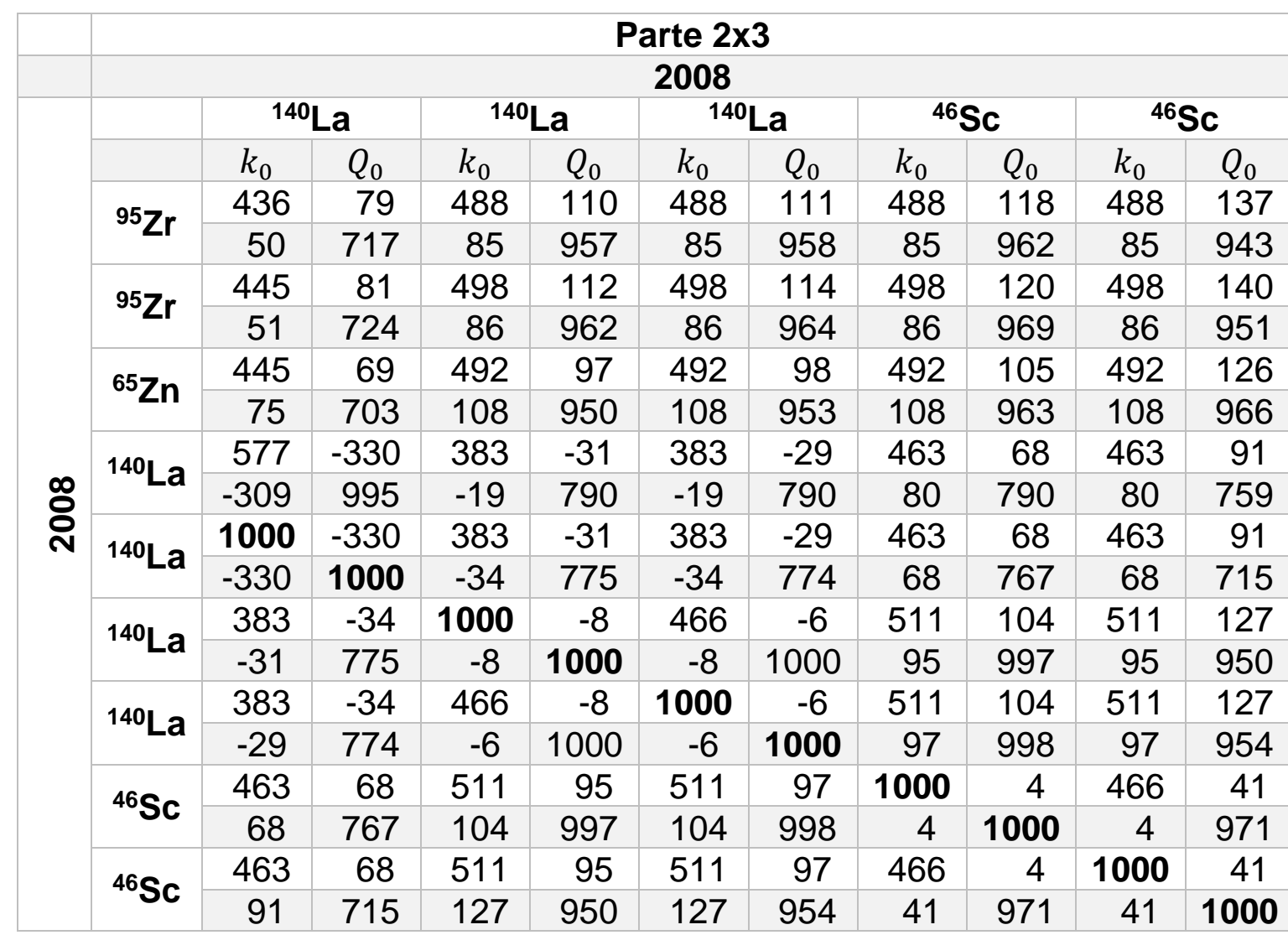




\section{Apêndice E}

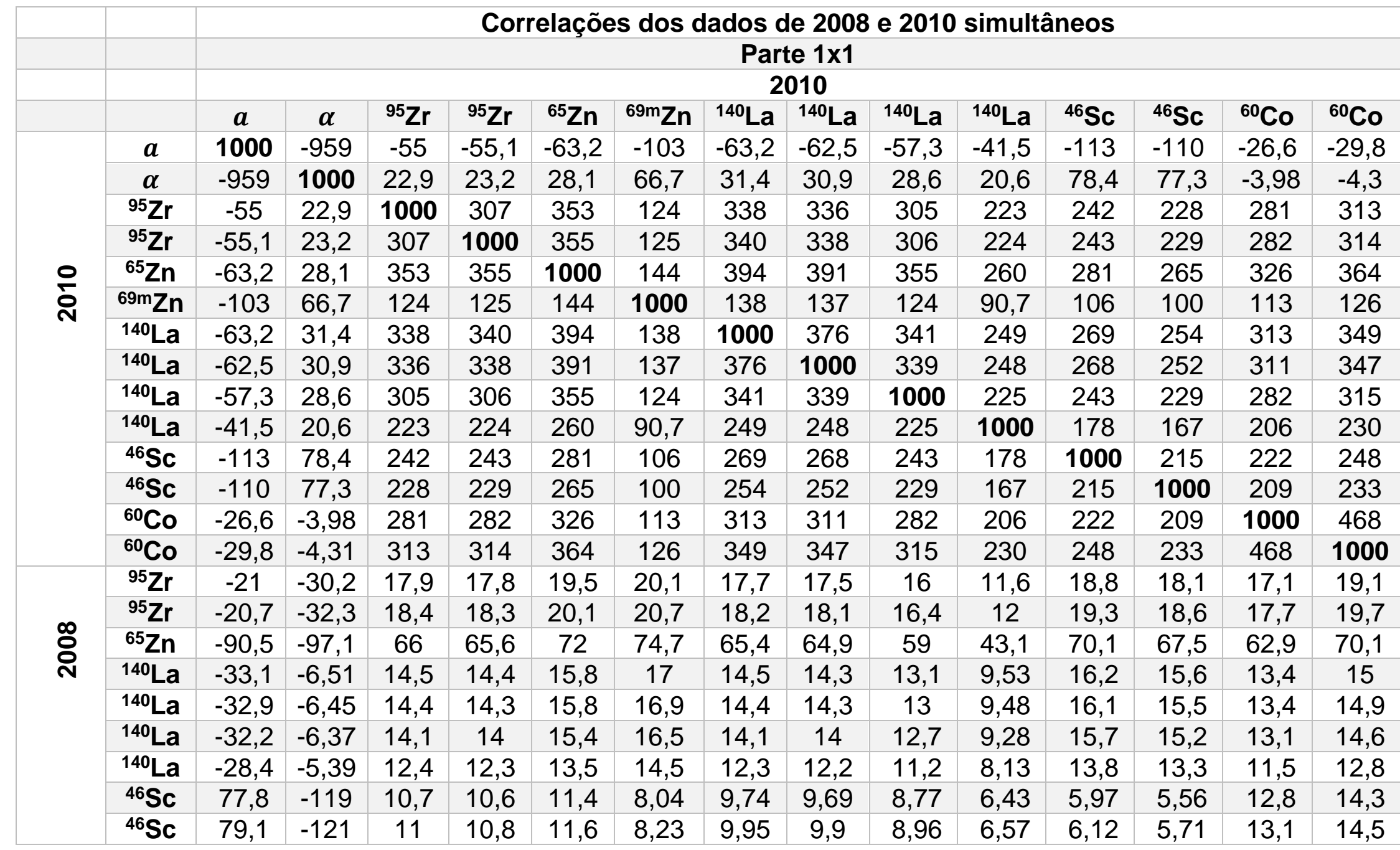


Correlações dos dados de 2008 e 2010 simultâneos Parte 1X2

\begin{tabular}{|c|c|c|c|c|c|c|c|c|c|c|}
\hline & & \multicolumn{9}{|c|}{ Parte $1 \times 2$} \\
\hline & & & & & & 2008 & & & & \\
\hline & & $95 \mathrm{Zr}$ & $95 \mathrm{Zr}$ & 65Zn & ${ }^{140} \mathrm{La}$ & ${ }^{140} \mathrm{La}$ & ${ }^{140} \mathrm{La}$ & ${ }^{140} \mathrm{La}$ & ${ }^{60} \mathrm{Co}$ & ${ }^{60} \mathrm{Co}$ \\
\hline \multirow{14}{*}{ 응 } & $a$ & -21 & $-20,7$ & $-90,5$ & $-33,1$ & $-32,9$ & $-32,2$ & $-28,4$ & 77,8 & 79,1 \\
\hline & $\alpha$ & $-30,2$ & $-32,3$ & $-97,1$ & $-6,51$ & $-6,45$ & $-6,37$ & $-5,39$ & -119 & -121 \\
\hline & ${ }^{95} \mathrm{Zr}$ & 17,9 & 18,4 & 66 & 14,5 & 14,4 & 14,1 & 12,4 & 10,7 & 11 \\
\hline & $95 \mathrm{Zr}$ & 17,8 & 18,3 & 65,6 & 14,4 & 14,3 & 14 & 12,3 & 10,6 & 10,8 \\
\hline & ${ }^{65} \mathrm{Zn}$ & 19,5 & 20,1 & 72 & 15,8 & 15,8 & 15,4 & 13,5 & 11,4 & 11,6 \\
\hline & $69 \mathrm{~m} \mathrm{Zn}$ & 20,1 & 20,7 & 74,7 & 17 & 16,9 & 16,5 & 14,5 & 8,04 & 8,23 \\
\hline & ${ }^{140} \mathrm{La}$ & 17,7 & 18,2 & 65,4 & 14,5 & 14,4 & 14,1 & 12,3 & 9,74 & 9,95 \\
\hline & ${ }^{140} \mathrm{La}$ & 17,5 & 18,1 & 64,9 & 14,3 & 14,3 & 14 & 12,2 & 9,69 & 9,9 \\
\hline & ${ }^{140} \mathrm{La}$ & 16 & 16,4 & 59 & 13,1 & 13 & 12,7 & 11,2 & 8,77 & 8,96 \\
\hline & ${ }^{140} \mathrm{La}$ & 11,6 & 12 & 43,1 & 9,53 & 9,48 & 9,28 & 8,13 & 6,43 & 6,57 \\
\hline & ${ }^{46} \mathrm{Sc}$ & 18,8 & 19,3 & 70,1 & 16,2 & 16,1 & 15,7 & 13,8 & 5,97 & 6,12 \\
\hline & ${ }^{46} \mathrm{Sc}$ & 18,1 & 18,6 & 67,5 & 15,6 & 15,5 & 15,2 & 13,3 & 5,56 & 5,71 \\
\hline & ${ }^{60} \mathrm{Co}$ & 17,1 & 17,7 & 62,9 & 13,4 & 13,4 & 13,1 & 11,5 & 12,8 & 13,1 \\
\hline & ${ }^{60} \mathrm{Co}$ & 19,1 & 19,7 & 70,1 & 15 & 14,9 & 14,6 & 12,8 & 14,3 & 14,5 \\
\hline \multirow{9}{*}{ 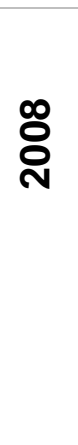 } & ${ }^{95} \mathrm{Zr}$ & 1000 & 345 & 307 & 351 & 348 & 336 & 272 & 239 & 246 \\
\hline & $95 \mathrm{Zr}$ & 345 & 1000 & 317 & 365 & 361 & 349 & 282 & 248 & 256 \\
\hline & ${ }^{65} \mathrm{Zn}$ & 307 & 317 & 1000 & 326 & 323 & 312 & 252 & 233 & 240 \\
\hline & ${ }^{140} \mathrm{La}$ & 351 & 365 & 326 & 1000 & 385 & 372 & 301 & 262 & 270 \\
\hline & ${ }^{140} \mathrm{La}$ & 348 & 361 & 323 & 385 & 1000 & 368 & 298 & 260 & 267 \\
\hline & ${ }^{140} \mathrm{La}$ & 336 & 349 & 312 & 372 & 368 & 1000 & 288 & 251 & 258 \\
\hline & ${ }^{140} \mathrm{La}$ & 272 & 282 & 252 & 301 & 298 & 288 & 1000 & 203 & 208 \\
\hline & ${ }^{46} \mathrm{Sc}$ & 239 & 248 & 233 & 262 & 260 & 251 & 203 & 1000 & 255 \\
\hline & ${ }^{46} \mathrm{Sc}$ & 246 & 256 & 040 & 270 & 267 & 258 & 209 & 255 & 1000 \\
\hline
\end{tabular}




\section{Apêndice F}

\begin{tabular}{|c|c|c|c|c|c|c|c|c|c|c|c|c|}
\hline & & \multicolumn{11}{|c|}{ Correlações dos dados de 2008 separados } \\
\hline & & $a$ & $\alpha$ & ${ }^{95} \mathrm{Zr}$ & $95 \mathrm{Zr}$ & ${ }^{65} \mathrm{Zn}$ & ${ }^{140} \mathrm{La}$ & ${ }^{140} \mathrm{La}$ & ${ }^{140} \mathrm{La}$ & ${ }^{140} \mathrm{La}$ & ${ }^{60} \mathrm{Co}$ & ${ }^{60} \mathrm{Co}$ \\
\hline \multirow{11}{*}{$\begin{array}{l}\infty \\
\text { ᄋ్ }\end{array}$} & $a$ & 1000 & -969 & -87.1 & -102 & -200 & -115 & -115 & -112 & -99.2 & 55 & 55.4 \\
\hline & $\alpha$ & -978 & 1000 & 2.96 & 1.48 & 21.5 & 20.5 & 20.4 & 19.9 & 17.9 & -132 & -134 \\
\hline & $95 \mathrm{Zr}$ & -87.1 & 2.96 & 1000 & 348 & 349 & 308 & 306 & 300 & 264 & 216 & 220 \\
\hline & ${ }^{95} \mathrm{Zr}$ & -102 & 1.48 & 348 & 1000 & 453 & 431 & 429 & 421 & 370 & 300 & 307 \\
\hline & ${ }^{65} \mathrm{Zn}$ & -200 & 21.5 & 349 & 453 & 1000 & 449 & 447 & 438 & 385 & 323 & 330 \\
\hline & ${ }^{140} \mathrm{La}$ & -115 & 20.5 & 308 & 431 & 449 & 1000 & 446 & 437 & 384 & 304 & 311 \\
\hline & ${ }^{140} \mathrm{La}$ & -115 & 20.4 & 306 & 429 & 447 & 446 & 1000 & 435 & 382 & 302 & 309 \\
\hline & ${ }^{140} \mathrm{La}$ & -112 & 19.9 & 300 & 421 & 438 & 437 & 435 & 1000 & 374 & 296 & 303 \\
\hline & ${ }^{140} \mathrm{La}$ & -99.2 & 17.9 & 264 & 370 & 385 & 384 & 382 & 374 & 1000 & 260 & 266 \\
\hline & ${ }^{60} \mathrm{Co}$ & 55 & -132 & 216 & 300 & 323 & 304 & 302 & 296 & 260 & 1000 & 313 \\
\hline & ${ }^{60} \mathrm{Co}$ & 55.4 & -134 & 220 & 307 & 330 & 311 & 309 & 303 & 266 & 313 & 1000 \\
\hline
\end{tabular}

\begin{tabular}{|c|c|c|c|c|c|c|c|c|c|c|c|c|c|c|c|}
\hline & & \multicolumn{14}{|c|}{ Correlações dos dados de 2010 separados } \\
\hline & & $\boldsymbol{a}$ & $\alpha$ & ${ }^{95} \mathrm{Zr}$ & $95 \mathrm{Zr}$ & $65 \mathrm{Zn}$ & 69m Zn & ${ }^{140} \mathrm{La}$ & ${ }^{140} \mathrm{La}$ & ${ }^{140} \mathrm{La}$ & ${ }^{140} \mathrm{La}$ & ${ }^{46} \mathrm{Sc}$ & ${ }^{46} \mathrm{Sc}$ & ${ }^{60} \mathrm{Co}$ & ${ }^{60} \mathrm{Co}$ \\
\hline \multirow{14}{*}{$\stackrel{ }{\circ}$} & $\boldsymbol{a}$ & 1000 & -930 & -54.7 & -54.9 & -63.5 & -113 & -65 & -64.3 & $-59,1$ & $-42,9$ & -125 & -123 & $-19,9$ & $-22,3$ \\
\hline & $\alpha$ & -930 & 1000 & -7.59 & -7.02 & -4.32 & 42.3 & 3.27 & 2.99 & 3,32 & 2,06 & 59,1 & 59,2 & $-39,5$ & $-43,9$ \\
\hline & ${ }^{95} \mathrm{Zr}$ & -54.7 & -7.59 & 1000 & 318 & 364 & 139 & 349 & 346 & 315 & 231 & 254 & 240 & 291 & 325 \\
\hline & ${ }^{95} \mathrm{Zr}$ & -54.9 & -7.02 & 318 & 1000 & 365 & 139 & 350 & 348 & 316 & 232 & 254 & 240 & 292 & 326 \\
\hline & ${ }^{65} \mathrm{Zn}$ & -63.5 & -4.32 & 364 & 365 & 1000 & 160 & 404 & 401 & 365 & 267 & 293 & 277 & 337 & 376 \\
\hline & $69 \mathrm{~m} \mathrm{Zn}$ & -113 & 42.3 & 139 & 139 & 160 & 1000 & 152 & 151 & 137 & 101 & 123 & 117 & 127 & 142 \\
\hline & ${ }^{140} \mathrm{La}$ & -65 & 3.27 & 349 & 350 & 404 & 152 & 1000 & 385 & 350 & 257 & 281 & 265 & 323 & 360 \\
\hline & ${ }^{140}$ La & -64.3 & 2.99 & 346 & 348 & 401 & 151 & 385 & 1000 & 347 & 255 & 279 & 264 & 321 & 357 \\
\hline & ${ }^{140} \mathrm{La}$ & -59.1 & 3.32 & 315 & 316 & 365 & 137 & 350 & 347 & 1000 & 232 & 254 & 239 & 291 & 324 \\
\hline & ${ }^{140} \mathrm{La}$ & -42.9 & 2.06 & 231 & 232 & 267 & 101 & 257 & 255 & 232 & 1000 & 186 & 176 & 214 & 238 \\
\hline & ${ }^{46} \mathrm{Sc}$ & -125 & 59.1 & 254 & 254 & 293 & 123 & 281 & 279 & 254 & 186 & 1000 & 228 & 233 & 259 \\
\hline & ${ }^{46} \mathrm{Sc}$ & -123 & 59.2 & 240 & 240 & 277 & 117 & 265 & 264 & 239 & 176 & 228 & 1000 & 220 & 245 \\
\hline & ${ }^{60} \mathrm{Co}$ & -19.9 & -39.5 & 291 & 292 & 337 & 127 & 323 & 321 & 291 & 214 & 233 & 220 & 1000 & 477 \\
\hline & ${ }^{60} \mathrm{Co}$ & -22.3 & -43.9 & 325 & 326 & 376 & 142 & 360 & 357 & 324 & 238 & 259 & 245 & 477 & 1000 \\
\hline
\end{tabular}




\section{Apêndice G}

Derivadas parciais para a montagem da matriz $V_{y}$. Para diminuir a quantidade de índices, abreviou-se as notações das grandezas:

$$
\begin{aligned}
& E_{i}=\left(\overline{E_{r, i}}\right), \\
& \left(A_{i}\right)_{C d}=\left(A_{s p, i}\right)_{C d}, \\
& \left(A_{i}\right)=\left(A_{s p, i}\right), \\
& \left(A_{A u}\right)_{C d}=\left(A_{s p, A u}\right)_{C d}, \\
& \left(A_{A u}\right)=\left(A_{s p, A u}\right), \\
& k_{0 i}=k_{0, A u}(i), \\
& \varepsilon_{i}=\varepsilon_{p, i}, \\
& \varepsilon_{A u}=\varepsilon_{p, A u}, \\
& F_{i}=F_{C d, i}, \\
& F_{A u}=F_{C d, A u}, \\
& G_{e i}=G_{e p, i}, \\
& G_{t i}=G_{t h, i}, \\
& G_{e A u}=G_{e p, A u}, \\
& G_{t A u}=G_{t h, A u} .
\end{aligned}
$$

\section{Covariâncias entre os elementos de $Y^{\text {sup }}$ do mesmo isótopo:}

$$
\begin{aligned}
V_{y}\left(Y_{i}^{\text {sup }}, Y_{j}^{\text {sup }}\right) & =\left(\frac{\partial Y_{i}^{\text {sup }}}{\partial Q_{0, i}}\right)\left(\frac{\partial Y_{j}^{\text {sup }}}{\partial Q_{0, j}}\right) \sigma_{Q_{0, i}}^{2}+ \\
& +\left(\frac{\partial Y_{i}^{\text {sup }}}{\partial F_{i}}\right)\left(\frac{\partial Y_{j}^{\text {sup }}}{\partial F_{j}}\right) \sigma_{F_{i}}^{2}+\left(\frac{\partial Y_{i}^{\text {sup }}}{\partial G_{e i}}\right)\left(\frac{\partial Y_{j}^{\text {sup }}}{\partial G_{e j}}\right) \sigma_{G_{e i}}^{2}+ \\
& +\left(\frac{\partial Y_{i}^{\text {sup }}}{\partial \varepsilon_{i}}\right)\left(\frac{\partial Y_{j}^{\text {sup }}}{\partial \varepsilon_{j}}\right) \operatorname{cov}\left(\epsilon_{i}, \epsilon_{j}\right)
\end{aligned}
$$

Se $i$ e $j$ são índices de transições gama do mesmo isótopo, o resultado é:

$$
V_{y}\left(Y_{i}^{\text {sup }}, Y_{j}^{\text {sup }}\right)=\frac{\sigma_{Q_{0, i}}^{2}}{Q_{0, i}^{2}}+\frac{\sigma_{F_{i}}^{2}}{F_{i}^{2}}+\frac{\sigma_{G_{e i}}^{2}}{G_{e i}^{2}}+\frac{\operatorname{cov}\left(\epsilon_{i}, \epsilon_{j}\right)}{\epsilon_{i} \cdot \epsilon_{j}} .
$$

Caso contrário:

$$
V_{y}\left(Y_{i}^{\text {sup }}, Y_{j}^{\text {sup }}\right)=0
$$


Covariâncias entre os elementos de $Y^{\text {sup }}$ e os elementos de $Y^{\text {inf }}$ da mesma transição gama:

$$
\begin{aligned}
V_{y}\left(Y_{i}^{\text {sup }}, Y_{j}^{\text {inf }}\right) & =\left(\frac{\partial Y_{i}^{\text {sup }}}{\partial\left(A_{i}\right)_{C d}}\right)\left(\frac{\partial Y_{j}^{\text {inf }}}{\partial\left(A_{j}\right)_{C d}}\right) \sigma_{\left(A_{i}\right)_{C d}}^{2}+ \\
& +\left(\frac{\partial Y_{i}^{\text {sup }}}{\partial F_{i}}\right)\left(\frac{\partial Y_{j}^{\text {inf }}}{\partial F_{j}}\right) \sigma_{F_{i}}^{2}+\left(\frac{\partial Y_{i}^{s u p}}{\partial \varepsilon_{i}}\right)\left(\frac{\partial Y_{j}^{\text {inf }}}{\partial \varepsilon_{j}}\right) \sigma_{\varepsilon_{i}}^{2}
\end{aligned}
$$

Cujo resultado é:

$$
\begin{aligned}
V_{y}\left(Y_{i}^{\text {sup }}, Y_{j}^{\text {inf }}\right) & =-\frac{\sigma_{\left(A_{i}\right)_{C d}}^{2}}{\left(A_{i}\right)_{C d} \cdot\left(A_{i} \cdot F_{i}-\left(A_{i}\right)_{C d}\right)}+ \\
& +\frac{\sigma_{F_{i}}^{2}}{F_{i}^{2} \cdot\left(A_{i} \cdot F_{i}-\left(A_{i}\right)_{C d}\right)}+\frac{\sigma_{\varepsilon_{i}}^{2}}{\varepsilon_{i}^{2}} .
\end{aligned}
$$

Covariâncias entre os elementos de $Y^{\text {sup }}$ e os elementos de $Y^{\text {inf }}$ do mesmo isótopo, mas de transições gama diferentes:

$$
V_{y}\left(Y_{i}^{\text {sup }}, Y_{j}^{\text {inf }}\right)=\left(\frac{\partial Y_{i}^{\text {sup }}}{\partial F_{i}}\right)\left(\frac{\partial Y_{j}^{\text {inf }}}{\partial F_{j}}\right) \sigma_{F_{i}}^{2}+\left(\frac{\partial Y_{i}^{s u p}}{\partial \varepsilon_{i}}\right)\left(\frac{\partial Y_{j}^{\text {inf }}}{\partial \varepsilon_{j}}\right) \operatorname{cov}\left(\epsilon_{i}, \epsilon_{j}\right)
$$

Cujo resultado é:

$$
V_{y}\left(Y_{i}^{\text {sup }}, Y_{j}^{\text {inf }}\right)=\frac{\sigma_{F_{j}}^{2}}{F_{j}^{2} \cdot\left(A_{j} \cdot F_{j}-\left(A_{j}\right)_{C d}\right)}+\frac{\operatorname{cov}\left(\epsilon_{i}, \epsilon_{j}\right)}{\epsilon_{i} \cdot \epsilon_{j}} .
$$


Covariâncias entre os elementos de $Y^{\text {inf }}$ do mesmo isótopo:

$$
\begin{aligned}
V_{y}\left(Y_{i}^{i n f}, Y_{j}^{i n f}\right) & =\left(\frac{\partial Y_{i}^{i n f}}{\partial\left(A_{A u}\right)_{C d}}\right)\left(\frac{\partial Y_{j}^{i n f}}{\partial\left(A_{A u}\right)_{C d}}\right) \sigma_{\left(A_{A u}\right)_{C d}}^{2}+ \\
& +\left(\frac{\partial Y_{i}^{i n f}}{\partial A_{A u}}\right)\left(\frac{\partial Y_{j}^{i n f}}{\partial A_{A u}}\right) \sigma_{A_{A u}}^{2}+\left(\frac{\partial Y_{i}^{i n f}}{\partial F_{A u}}\right)\left(\frac{\partial Y_{j}^{i n f}}{\partial F_{A u}}\right) \sigma_{F_{A u}}^{2}+ \\
& +\left(\frac{\partial Y_{i}^{i n f}}{\partial G_{t A u}}\right)\left(\frac{\partial Y_{j}^{i n f}}{\partial G_{t A u}}\right) \sigma_{G_{t A u}}^{2}+\left(\frac{\partial Y_{i}^{i n f}}{\partial \varepsilon_{A u}}\right)\left(\frac{\partial Y_{j}^{i n f}}{\partial \varepsilon_{A u}}\right) \sigma_{\varepsilon_{A u}}^{2}+ \\
& +\left(\frac{\partial Y_{i}^{i n f}}{\partial G_{t i}}\right)\left(\frac{\partial Y_{j}^{i n f}}{\partial G_{t j}}\right) \sigma_{G_{t i}}^{2}+\left(\frac{\partial Y_{i}^{i n f}}{\partial F_{i}}\right)\left(\frac{\partial Y_{j}^{i n f}}{\partial F_{j}}\right) \sigma_{F_{i}}^{2}+ \\
& +\left(\frac{\partial Y_{i}^{i n f}}{\partial \varepsilon_{i}}\right)\left(\frac{\partial Y_{j}^{i n f}}{\partial \varepsilon_{j}}\right) \operatorname{cov}\left(\epsilon_{i}, \epsilon_{j}\right),
\end{aligned}
$$

Cujo resultado é:

$$
\begin{aligned}
V_{y}\left(Y_{i}^{i n f}, Y_{j}^{i n f}\right) & =\frac{\sigma_{\left(A_{A u}\right)_{C d}}^{2}}{\left(A_{A u} \cdot F_{A u}-\left(A_{A u}\right)_{C d}\right)^{2}}+\frac{\sigma_{A_{A u}}^{2}}{\left(A_{A u}-\frac{\left(A_{A u}\right)_{C d}}{F_{A u}}\right)^{2}}+ \\
& +\frac{\left(A_{A u}\right)_{C d} \cdot \sigma_{F_{A u}}^{2}}{\left(A_{A u} \cdot F_{A u}^{2}-\left(A_{A u}\right)_{C d} \cdot F_{A u}\right)^{2}}+\frac{\sigma_{G_{t A u}}^{2}}{G_{t A u}^{2}}+\frac{\sigma_{\varepsilon_{A u}}^{2}}{\varepsilon_{A u}^{2}}+\frac{\sigma_{G_{t i}}^{2}}{G_{t i}^{2}}+ \\
& +\frac{\left(A_{i}\right)_{C d} \cdot\left(A_{j}\right)_{C d} \cdot \sigma_{F_{i}}^{2}}{F_{i}^{2}\left(A_{i} \cdot F_{i}-\left(A_{i}\right)_{C d}\right)\left(A_{j} \cdot F_{i}-\left(A_{j}\right)_{C d}\right)}+ \\
& +\frac{\operatorname{cov}\left(\epsilon_{i}, \epsilon_{j}\right)}{\epsilon_{i} \cdot \epsilon_{j}}
\end{aligned}
$$


Covariâncias entre os elementos de $Y^{\text {inf }}$ de isótopos diferentes:

$$
\begin{aligned}
V_{y}\left(Y_{i}^{i n f}, Y_{j}^{i n f}\right) & =\left(\frac{\partial Y_{i}^{i n f}}{\partial\left(A_{A u}\right)_{C d}}\right)\left(\frac{\partial Y_{j}^{i n f}}{\partial\left(A_{A u}\right)_{C d}}\right) \sigma_{\left(A_{A u}\right)_{C d}}^{2} \\
& +\left(\frac{\partial Y_{i}^{i n f}}{\partial A_{A u}}\right)\left(\frac{\partial Y_{j}^{i n f}}{\partial A_{A u}}\right) \sigma_{A_{A u}}^{2}+\left(\frac{\partial Y_{i}^{i n f}}{\partial F_{A u}}\right)\left(\frac{\partial Y_{j}^{i n f}}{\partial F_{A u}}\right) \sigma_{F_{A u}}^{2}+ \\
& +\left(\frac{\partial Y_{i}^{i n f}}{\partial G_{t A u}}\right)\left(\frac{\partial Y_{j}^{i n f}}{\partial G_{t A u}}\right) \sigma_{G_{t A u}}^{2}+\left(\frac{\partial Y_{i}^{i n f}}{\partial \varepsilon_{A u}}\right)\left(\frac{\partial Y_{j}^{i n f}}{\partial \varepsilon_{A u}}\right) \sigma_{\varepsilon_{A u}}^{2}+ \\
& +\left(\frac{\partial Y_{i}^{i n f}}{\partial \varepsilon_{i}}\right)\left(\frac{\partial Y_{j}^{i n f}}{\partial \varepsilon_{j}}\right) \operatorname{cov}\left(\epsilon_{i}, \epsilon_{j}\right)+\left(\frac{\partial Y_{i}^{i n f}}{\partial \varepsilon_{i}}\right)\left(\frac{\partial Y_{j}^{i n f}}{\partial \varepsilon_{A u}}\right) \operatorname{cov}\left(\epsilon_{i}, \epsilon_{A u}\right)+ \\
& +\left(\frac{\partial Y_{i}^{i n f}}{\partial \varepsilon_{A u}}\right)\left(\frac{\partial Y_{j}^{i n f}}{\partial \varepsilon_{j}}\right) \operatorname{cov}\left(\epsilon_{A u}, \epsilon_{j}\right),
\end{aligned}
$$

Cujo resultado é:

$$
\begin{aligned}
V_{y}\left(Y_{i}^{i n f}, Y_{j}^{i n f}\right) & =\frac{\sigma_{\left(A_{A u}\right)_{C d}}^{2}}{\left(A_{A u} \cdot F_{A u}-\left(A_{A u}\right)_{C d}\right)^{2}}+\frac{\sigma_{A_{A u}}^{2}}{\left(A_{A u}-\frac{\left(A_{A u}\right)_{C d}}{F_{A u}}\right)^{2}}+ \\
& +\frac{\left(A_{A u}\right)_{C d} \cdot \sigma_{F_{A u}}^{2}}{\left(A_{A u} \cdot F_{A u}^{2}-\left(A_{A u}\right)_{C d} \cdot F_{A u}\right)^{2}}+\frac{\sigma_{G_{t A u}}^{2}}{G_{t A u}^{2}}+\frac{\sigma_{\varepsilon_{A u}}^{2}}{\varepsilon_{A u}^{2}}+ \\
& +\frac{\operatorname{cov}\left(\epsilon_{i}, \epsilon_{j}\right)}{\epsilon_{i} \cdot \epsilon_{j}}+\frac{\operatorname{cov}\left(\epsilon_{i}, \epsilon_{A u}\right)}{\epsilon_{i} \cdot \epsilon_{A u}}+\frac{\operatorname{cov}\left(\epsilon_{A u}, \epsilon_{j}\right)}{\epsilon_{A u} \cdot \epsilon_{j}} .
\end{aligned}
$$




\section{REFERÊNCIAS BIBLIOGRÁFICAS}

[1] GUINN, V. P., HOSTE, J., "Neutron Activation Analysis," Elem. Anal. Biol. Mater., p. 382, 1980.

[2] GUINN, V. P., "Nuclear activation analysis; 45 years after Geroge Hevesy's discovery," J. Radioanal. Nucl. Chem., vol. 59, no. 2, pp. 309-314, 1980.

[3] L'ANNUNZIATA, M. F., Radioactivity: introduction and history. Elsevier, 2007.

[4] ROSSBaCh, M., StOeppleR, M., BYRNE, A. R., "Prompt and delayed NAA techniques for the characterization of specimen bank materials," Sci. Total Environ., vol. 139, no. 140, p. 411/419, 1993.

[5] BEREZNAI, T., "Methods, problems and trends of standardization in multielement reactor neutron activation analysis," Fresenius' Zeitschrift fur Anal. Chemie, vol. 302, no. 5, pp. 353-363, 1980.

[6] KUBEŠOVÁ, M., KUČERA, J., " $k_{0}$ standardization in neutron activation analysis at LVR-15 reactor in Ř e ž," Czech Technical University in Prague, 2012.

[7] KAFALA, S. I., MACMAHON, T. D., "Comparison of neutron activation analysis methods," J. Radioanal. Nucl. Chem., vol. 271, no. 2, pp. 507-516, Feb. 2007.

[8] FRONTASYEVA, M. V., "Neutron activation analysis in the life sciences," Phys. Part. Nucl., vol. 42, no. 2, pp. 332-378, Mar. 2011.

[9] DIAS, M. S., CARDOSO, V., KOSKINAS, M. F., YAMAZAKI, I. M., "Determination of the neutron spectrum shape parameter $\alpha$ in $k_{0}$ NAA methodology using covariance analysis," Appl. Radiat. Isot., vol. 68, no. 4-5, pp. 592-595, Apr. 2010.

[10] DUNG, H. M., HIEN, P. D., "The application and development of $k_{0}$ standardization method of neutron activation analysis at Dalat research reactor," J. Radioanal. Nucl. Chem., vol. 257, no. 3, pp. 643-647, Sep. 2003.

[11] DE CORTE, F., "The $k_{0}$-Standartdization Method: A move to the optimization of neutron activation analysis," Rijksuniversiteit Gent, 1987.

[12] SMODIŠ, B., BUČAR, T., "Overall measurement uncertainty of $k_{0}$-based neutron activation analysis," J. Radioanal. Nucl. Chem., vol. 269, no. 2, pp. 
311-316, Aug. 2006.

[13] CRARY, M. A., MANN, G. D. C., GROHER, M. E., "Initial psychometric assessment of a functional oral intake scale for dysphagia in stroke patients.," Arch. Phys. Med. Rehabil., vol. 86, no. 8, pp. 1516-20, Aug. 2005.

[14] BIRGÜL, O., DIKŠIĆ, M., YAFFE, L., "A comparison of the statistical treatment of results using concentrations of elements determined by neutron activation and X-ray fluorescence analysis methods," J. Radioanal. Chem., vol. 55, no. 1, pp. 101-109, Mar. 1980.

[15] BENEDIK, L., REPINC, U., "Evaluation of measurement uncertainty components associated with results of radiochemical neutron activation analysis for determination of uranium traces," Accredit. Qual. Assur., vol. 16, no. 12, pp. 637-642, Dec. 2011.

[16] ROBOUCH, P., ARANA, G., EGUSKIZA, M., POMMÉ, S., ETXEBARRIA, N., "Uncertainty Budget for $k_{0}$-NAA," J. Radioanal. Nucl. Chem., vol. 245, no. 1, pp. 195-197, 2000.

[17] DIAS, M. S., CARDOSO, V., KOSKINAS, M. F., YAMAZAKI, I. M., SEMMLER, R., MORALLES, M., ZAHN, G. S., GENEZINI, F. A., DE MENEZES, M. O., FIGUEIREDO, A. M. G., "Measurements of $k_{0}$ and $Q_{0}$ values for ${ }^{64} \mathrm{Zn}(\mathrm{n}, \mathrm{y})^{65} \mathrm{Zn}$ and ${ }^{68} \mathrm{Zn}(\mathrm{n}, \mathrm{\gamma})^{69 \mathrm{~m} Z \mathrm{n}}$ reactions with covariance analysis," Appl. Radiat. Isot., vol. 69, no. 7, pp. 960-964, Jul. 2011.

[18] CARDOSO, V., "Estudo das covariâncias envolvidas no método $k_{0}$ de análise por ativação neutrônica," Universidade de São Paulo, 2011.

[19] MORALLES, M., "Método $k_{0}$, um procedimento de ajuste linear nos parâmetros para o cálculo simultâneo de $\alpha$ e $k_{0}$." Relatório interno do Centro de Reator de Pesquisas (CRPq), IPEN-CNEN, São Paulo, 2010.

[20] HELENE, O., Metodos dos Minimos Quadrados. São Paulo: Editora Livraria da Física, 2006.

[21] KRANE, K. S., HALLIDAY, D., Introductory nuclear physics, vol. 465. Wiley New York, 1988.

[22] KNOLL, G. F., Radiation detection and measurement, 4th ed. Michigan: John Wiley \& Sons, 2010.

[23] TERREMOTO, L. A. A., "Disciplina TNR5764 - Fundamentos de Tecnologia Nuclear - Reatores," 2004. [Online]. Available: http://stoa.usp.br/turin/files/- 
1/16586/TNR5764-AP.pdf. [Accessed: 15-Feb-2017].

[24] DE CORTE, F., MOENS, L., SORDO-EL HAMMAMI, K., SIMONITS, A., HOSTE, J., "Modification and generalization of some methods to improve the accuracy of $\alpha$-determination in the $1 / E 1+\alpha$ epithermal neutron spectrum," J. Radioanal. Chem., vol. 52, no. 2, pp. 305-317, Sep. 1979.

[25] YÜCEL, H., KARADAG, M., "Experimental determination of the $\alpha$-shape factor in the $1 / \mathrm{E} 1+\alpha$ epithermal-isotopic neutron source-spectrum by dual monitor method," Ann. Nucl. Energy, vol. 31, no. 6, pp. 681-695, 2004.

[26] FERREIRA JÚNIOR, F. A., "Medida da secção de choque térmica e da integral de ressonância da reação 41-K (n,y) 42-K," 2008. [Online]. Available:

https://www.google.com.br/url?sa=t\&rct=j\&q=\&esrc=s\&source=web\&cd=1\&c $\mathrm{ad}=\mathrm{rja} \&$ uact $=8 \&$ ved $=0$ ahUKEwi35Jvp05DSAhVi2oMKHS70DyEQFggcMAA \&url=http\%3A\%2F\%2Fwww.teses.usp.br\%2Fteses\%2Fdisponiveis\%2F43\% 2F43134\%2Ftde-03112008-

104617\%2Fpublico\%2FFelisbertoAlves.pdf\&usg=A. [Accessed: 14-Feb2017].

[27] BECKURTS, K.-H., WIRTZ, K., Neutron physics. Springer Science \& Business Media, 2013.

[28] BLATT, J. M., WEISSKOPF, V. F., Theoretical nuclear physics. Springer Science \& Business Media, 2012.

[29] GLENDENNING, N., Direct nuclear reactions. Elsevier, 2012.

[30] GIBSON, W. M., "The physics of nuclear reactions," 1980.

[31] HØGDAHL, O. T., "Neutron absorption in pile neutron activation analysis," Michigan. Univ., Ann Arbor. Michigan Memorial-Phoenix Project, 1962.

[32] DE CORTE, F., BELLEMANS, F., NEVE, P., SIMONITS, A., "The use of a modified Westcott-formalism in the $k_{0}$-standardization of NAA: The state of affairs," J. Radioanal. Nucl. Chem., vol. 179, no. 1, pp. 93-103, 1994.

[33] HOLDEN, N. E., "Temperature Dependence of the Westcott g-factor for Neutron Reactions in Activation Analysis," Pure Appl. Chem., vol. 71, no. 12, pp. 2309-2315, Jan. 1999.

[34] WEStCOTT, C. H., WALKER, W. H., ALEXANDER, T. K., "Effective cross sections and cadmium ratios for the neutron spectra of thermal reactors," Second United Nations Int. Conf. Peac. Uses At. Energy, no. 1, 1958. 
[35] HAMIDATOU, L., RAMDHANE, M., "Characterization of neutron spectrum at Es-Salam Research Reactor using Hogdahl convention and Westcott formalism for the $k_{0}$-based neutron activation analysis," J. Radioanal. Nucl. Chem., vol. 278, no. 3, pp. 627-630, 2008.

[36] VAN SLUIJS, R., JAĆIMOVIĆ, R., KENNEDY, G., "A simplified method to replace the Westcott formalism in $k_{0}$-NAA using non-1/v nuclides," $J$. Radioanal. Nucl. Chem., vol. 300, no. 2, pp. 539-545, 2014.

[37] WESTCOTT, C. H., "Effective cross section values for well-moderated thermal reactor spectra," AECL--1101-REV, 1970. [Online]. Available: http://www.iaea.org/inis/collection/NCLCollectionStore/_Public/41/057/41057 250.pdf. [Accessed: 21-Feb-2017].

[38] SCHMIDT, P. F., GLASCOCK, M. D., "Applications and Problems of Parametric Counting," J. Radioanal. Chem., vol. 72, no. 1, pp. 231-244, 1982.

[39] SIMONITS, A., DE CORTE, F., MOENS, L., HOSTE, J., "Status and recent developments in the $k_{0}$-standardization method," J. Radioanal. Chem., vol. 72, no. 1-2, pp. 209-230, 1982.

[40] DE CORTE, F., SIMONITS, A., BellemANS, F., FREITAS, M. C., JOVANOVIĆ, S., SMODIŠ, B., ERDTMANN, G., PETRI, H., DE WISPELAERE, A., "Recent advances in the $k_{0}$-standardization of neutron activation analysis: Extensions, applications, prospects," J. Radioanal. Nucl. Chem. Artic., vol. 169, no. 1, pp. 125-158, 1993.

[41] YAVAR, A., SARMANI, S., WOOD, A. K., ZAINAL, N. S., KHOO, K. S., "Development and implementation of Hogdahl convention and Westcott formalism for $k_{0}$-INAA application at Malaysian Nuclear Agency reactor," J. Radioanal. Nucl. Chem., vol. 291, no. 2, pp. 521-527, 2012.

[42] HAMidATOU, L., Slamene, H., AKHAL, T., ZOURANE, B., "Concepts, Instrumentation and Techniques of Neutron Activation Analysis," in Imaging and Radioanalytical Techniques in Interdisciplinary Research Fundamentals and Cutting Edge Applications, InTech, 2013.

[43] VERHEIJKE, M. L., "Relation between the Hogdahl convention and the modified Westcott formalism for $(\mathrm{n}, \alpha)$ reactions with a pure 1/vn crosssection behavior," J. Radioanal. Nucl. Chem. Artic., vol. 183, no. 2, pp. 293299, 1994. 
[44] BLAAUW, M., BODE, P., DE BRUIN, M., "An alternative convention describing the (n, $\alpha)$-reaction rate suited for use in the $k_{0}$-method of NAA," $J$. Radioanal. Nucl. Chem. Artic., vol. 152, no. 2, pp. 435-445, 1991.

[45] RYVES, T. B., "A new thermal neutron flux convention," Metrologia, vol. 5, no. 4, p. 119, 1969.

[46] StOUGHTON, R. W., HALPERIN, J., "Effective Cutoff Energies for Boron, Cadmium, Gadolinium, and Samarium Filters," Eff. Cutoff Energies Boron, Cadmium, Gadolinium, Samarium Filters, vol. 15, no. 3, pp. 314-324, 1963.

[47] HANCOCK, R., "Neutron Activation Analysis," in Encyclopedia of Scientific Dating Methods, Dordrecht: Springer Netherlands, 2013, pp. 1-2.

[48] PINO, F., SAJO-CASTELLI, A. M., BARROS, H., VERMAERCKE, P., SNEYERS, L., SAJO BOHUS, L., MACKOWIAK DE ANTCZAK, M. M., ANTCZAK, A., " $k_{0}$-INAA of Venezuelan ceramics and complete statistical analysis to establish their provenance," J. Radioanal. Nucl. Chem., vol. 298, no. 2, pp. 1257-1272, Nov. 2013.

[49] GLASCOCK, M. D., "Neutron Activation Analysis (NAA): Applications in Archaeology BT - Encyclopedia of Global Archaeology," C. Smith, Ed. New York, NY: Springer New York, 2014, pp. 5239-5247.

[50] BALLA, M., KEOMLEY, G., ROSNER, G., "Neutron activation analysis for provenance studies of archaeological ceramics," J. Radioanal. Nucl. Chem., vol. 141, no. 1, pp. 7-16, 190AD.

[51] CILIBERTO, E., SPOTO, G., Modern analytical methods in art and archaeology. John Wiley \& Sons Canada, 2000.

[52] NETZEL, D. A., STANLEY, C. W., RATHBURN, D. W., "A neutron activation method for soil removal measurements: A comparison of the reflectance method and the neutron activation method," J. Am. Oil Chem. Soc., vol. 41, no. 10, pp. 678-683, Oct. 1964.

[53] KEHAYIAS, J. J., "Evaluation of new pharmaceuticals using in vivo neutron inelastic scattering and neutron activation analysis," J. Radioanal. Nucl. Chem., vol. 244, no. 1, pp. 219-224, 2000.

[54] DUTOV, A. G., KOMAR, V. A., SHIRYAEV, S. V., SMAKHTIN, L. A., "Neutron-activation analysis of the impurity composition of gallium arsenide based semiconductor structures," Semiconductors, vol. 31, no. 4, pp. 415417, Apr. 1997. 
[55] JAĆIMOVIĆ, R., LAZARU, A., MIHAJLOVI, D., ILI, R., "Determination of major and trace elements in some minerals by $k_{0}$-instrumental neutron activation analysis," J. Radioanal. Nucl. Chem., vol. 253, no. 3, pp. 427-434, 2002.

[56] LAZARUS, C. L., LOGEMANN, J. A., PAULOSKI, B. R., COLANGELO, L. A., KAHRILAS, P. J., MITTAL, B. B., PIERCE, M., "Swallowing Disorders in Head and Neck Cancer Patients Treated With Radiotherapy and Adjuvant Chemotherapy," Laryngoscope, vol. 106, no. 9, pp. 1157-1166, Sep. 1996.

[57] MADDOCK, R. S., MEINKE, W. W., Activation Analysis; Nuclear Chemical Research; Radiochemical Separations, 1st ed. Michigan: University of Michigan, 1959.

[58] FRANCO, M. B., SABINO, C. de V. S., "Determinação dos Parmâmetros Nucleares $\alpha, f$ e Temperatura de Nêutrons no Reator Triga Mark I IPR R1," 2011.

[59] KIM, J. I., "Monostandard activation analysis: evaluation of the method and its accuracy," J. Radioanal. Chem., vol. 63, no. 1, pp. 121-144, 1981.

[60] SIMONITS, A., DE CORTE, F., HOSTE, J., "Single Comparator Methods in Reactor Neutron Activation Analysis," J. Radioanal. Chem., vol. 24, pp. 3146, 1975.

[61] GIRARDI, F., GUZZI, G., PAULY, J., "Reactor Neutron Activation Analysis by the Single Comparator Method.," Anal. Chem., vol. 37, no. 9, pp. 10851092, Aug. 1965.

[62] DE CORTE, F., SPEECKE, A., HOSTE, J., "Reactor neutron activation analysis by a triple comparator method," J. Radioanal. Chem., vol. 3, no. 34, pp. 205-215, Sep. 1969.

[63] DE CORTE, F., SIMONITS, A., Vade Mecum for $k_{0}$ - Users, 3rd ed. Geleen: DSM Research, 1994.

[Online].

Available:

https://www.ipen.br/biblioteca/cd/inac/2002/ENAN/E02/E02_365.PDF. [Accessed: 12-Apr-2017].

[64] DE CORTE, F., hamMAMI, K. S.-E., MOENS, L., SIMONITS, A., WISPELAERE, A., HOSTE, J., "The accuracy and precision of the experimental $\alpha$-determination in the $1 / \mathrm{E} 1+\alpha$ epithermal reactor-neutron spectrum," J. Radioanal. Chem., vol. 62, no. 1-2, pp. 209-255, Mar. 1981. 
[65] KODELI, I., TRKOV, A., "Validation of the IRDF-2002 dosimetry library," Nucl. Instruments Methods Phys. Res. Sect. A Accel. Spectrometers, Detect. Assoc. Equip., vol. 577, no. 3, pp. 664-681, Jul. 2007.

[66] TSOULFANIDIS, N., Measurement and detection of radiation. CRC press, 2013.

[67] DIAS, M. S., TAKEDA, M. N., KOSKINAS, M. F., "Cascade summing corrections for HPGe spectrometers by the Monte Carlo method," Appl. Radiat. Isot., vol. 56, no. 1-2, pp. 105-109, 2002.

[68] GONÇALVES, I. F., MARTINHO, E., SALGADO, J., "Monte Carlo calculation of epithermal neutron resonance self-shielding factors in foils of different materials," Appl. Radiat. Isot., vol. 56, no. 6, pp. 945-951, 2002.

[69] MARTINHO, E., GONÇALVES, I. F., SALGADO, J., "Universal curve of epithermal neutron resonance self-shielding factors in foils, wires and spheres," Appl. Radiat. Isot., vol. 58, no. 3, pp. 371-375, 2003.

[70] MARTINHO, E., SALGADO, J., GONÇALVES, I. F., "Universal curve of the thermal neutron self-shielding factor in foils, wires, spheres and cylinders," $J$. Radioanal. Nucl. Chem., vol. 261, no. 3, pp. 637-643, 2004.

[71] BLAAUW, M., "The confusing issue of the neutron capture cross-section to use in thermal neutron self-shielding computations," Nucl. Inst. Methods Phys. Res. A, vol. 356, no. 2-3, pp. 403-407, 1995.

[72] TAYLOR, J., Introduction to error analysis, the study of uncertainties in physical measurements, 2nd ed., vol. 1. Colorado, 1997.

[73] KUBEŠOVÁ, M., KUČERA, J., "How to calculate uncertainties of neutron flux parameters and uncertainties of analysis results in $k_{0}$-NAA?," J. Radioanal. Nucl. Chem., vol. 293, no. 1, pp. 87-94, Jul. 2012.

[74] KUČERA, J., BODE, P., STEPANEK, V., "The 1993 ISO Guide to the expression of uncertainty in measurement applied to NAA," J. Radioanal. Nucl. Chem., vol. 245, no. 1, pp. 115-122, 2000.

[75] YOUNES, N., ROBOUCH, P., "A Web tool for calculating $k_{0}-\mathrm{NAA}$ uncertainties," J. Radioanal. Nucl. Chem., vol. 257, no. 3, pp. 665-667, 2003.

[76] KUBEŠOVÁ, M., KUČERA, J., "Comparison of Kayzero for Windows and $k_{0}$ IAEA software packages for $k_{0}$ standardization in neutron activation analysis," Nucl. Instruments Methods Phys. Res. Sect. A Accel. 
Spectrometers, Detect. Assoc. Equip., vol. 654, no. 1, pp. 206-212, Oct. 2011.

[77] ROSSBACH, M., BLAAUW, M., BACCHI, M. A., LIN, X., "The $k_{0}$-IAEA program," J. Radioanal. Nucl. Chem., vol. 274, no. 3, pp. 657-662, 2007.

[78] IAEA, " $k_{0}$-IAEA manual," IAEA-Vienna, 2014. [Online]. Available: http://wwwnaweb.iaea.org/napc/iachem/K0-IAEA.html. [Accessed: 24-Apr-2017].

[79] EUROPEAN COMPUTER MANUFACTURERS ASSOCIATION, "ECMA Standard on FORTRAN," 1965. [Online]. Available: http://www.ecmainternational.org/publications/files/ECMA-ST-WITHDRAWN/ECMA-9, 1st Edition, April 1965.pdf. [Accessed: 06-Jul-2017].

[80] ORENGO, G., Fortran 95: Curso básico, 1st ed. Santa Maria-RS, 2016.

[81] GNU, "GNU Fortran," 2017. [Online]. Available: https://gcc.gnu.org/fortran/. [Accessed: 06-Jul-2017].

[82] CYGWIN, "Cygwin Project," 2017. [Online]. Available: https://www.cygwin.com/. [Accessed: 06-Jul-2017].

[83] ECLIPSE COMMUNIT, "Eclipse," 2017. [Online]. Available: https://www.eclipse.org/org/. [Accessed: 06-Jul-2017].

[84] SCILAB ENTERPRISES, "Scilab," 2017. [Online]. Available: http://www.scilab.org/scilab/about. [Accessed: 06-Jul-2017].

[85] DIAS, M. S., "Programa Alpino V. 1 - Manual do Usuário." Centro do Reator de Pesquisas, IPEN-CNEN, São Paulo, 2001.

[86] BÉ,M.M., CHISTÉ,V., DULIEU,D., BROWNE,E, CHECHEV,V., KUZMENKO,N., HELMER,R., NICHOLSS, A., SCHONFIELD,E., DERSCH,R., Monographic BIPM-5-Table of Radionuclides. Volumes 1 e 2, 2004

[87] IAEA - Calibration Certificate, Reference date: 1/01/1981

[88] IUPAC, DE CORTE, F., KOLOTOV, V. P., "Compilation of $k_{0}$ and Related Data for NAA, versão 4," International Union of Pure and Applied Chemistry. Acces Electronic Database, 2002. [Online]. Available: http://old.iupac.org/projects/2001/2001-075-1-500.html. [Accessed: 05-May2017]. 